Supporting Information

\title{
Ultrafast Energy Transfer From Local Exciton To Intermolecular CT States In A Supramolecular Model Of The Donor-Acceptor Interface
}

Loïc Tanguy ${ }^{\dagger}$, Alexandre fleury $^{\dagger}$, Paul-Ludovic Karsenti ${ }^{\dagger}$, Gessie Brisard ${ }^{\dagger}$, Armand Soldera $^{\dagger}$ and Pierre D. Harvey ${ }^{\dagger *}$

'Department of Chemistry, University of Sherbrooke, 2500 Boulevard de l'Université, Sherbrooke, Quebec J1K 2R1, Canada

*E-mail: Pierre.Harvey@usherbrooke.ca (Prof. Pierre D. Harvey) 


\section{Table of content}

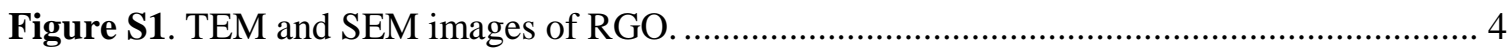

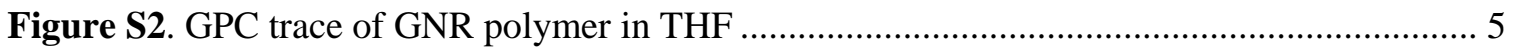

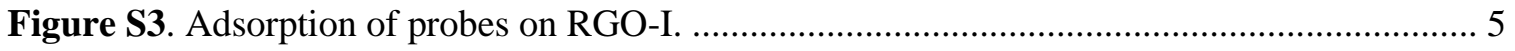

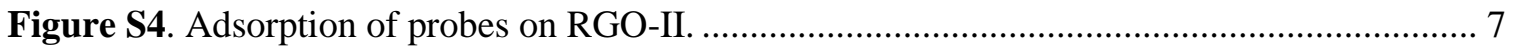

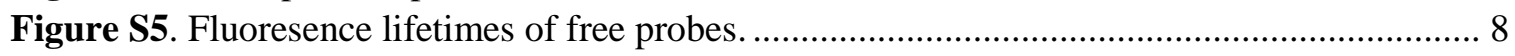

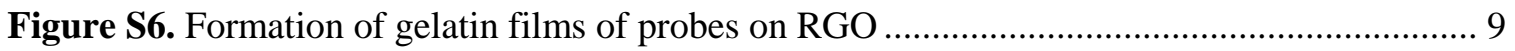

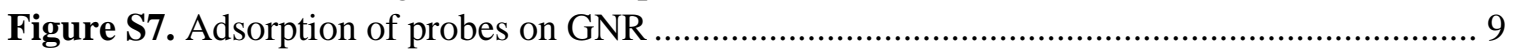

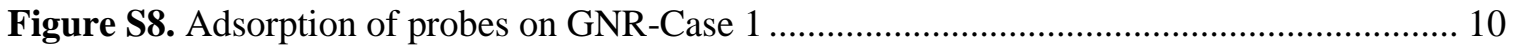

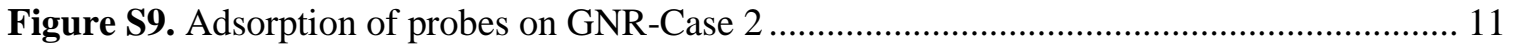

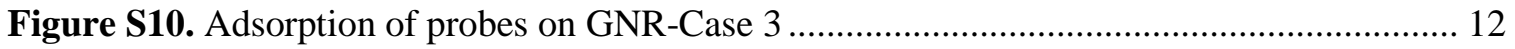

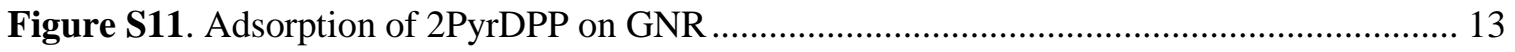

Figure S12. Predicted absorption of the 2PyrDPP-GNR CT assembly by predicted by DFT...... 14

Figure S13. Representations of the HOMO-2, HOMO-1, LUMO+1 and LUMO+2 for the

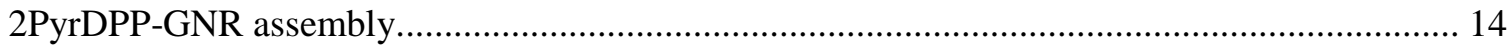

Figure S14. Equilibrium between adsorbed and free probes ................................................. 16

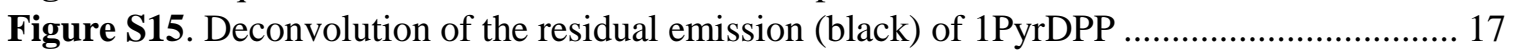

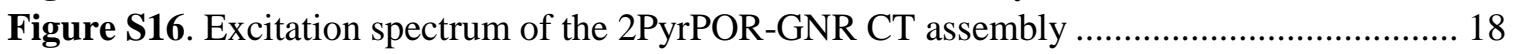

Figure S17. Fluorescence quantum yields of GNR and CT states............................................ 19

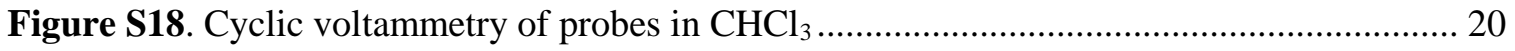

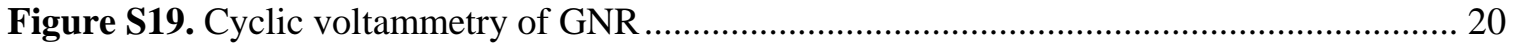

Figure S20. Absorption spectra of the 2PyrPOR, 2PyrDPP and 2PyrPBI/GNR CT assemblies

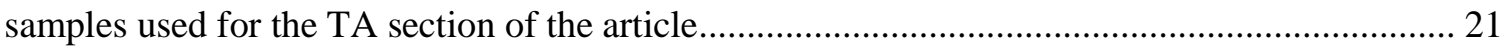

Figure S21. Time-evolution of the GSB band of GNR* for the six CT assemblies adsorbed on

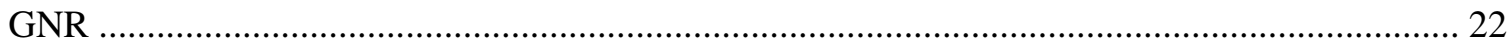

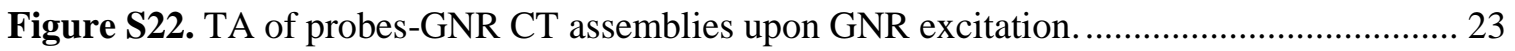

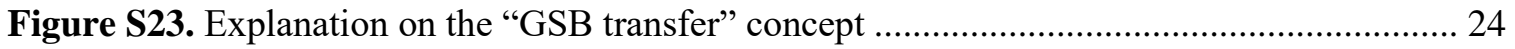

Figure S24. Time evolution of the GNR GSB and CT complex GSB intensities of

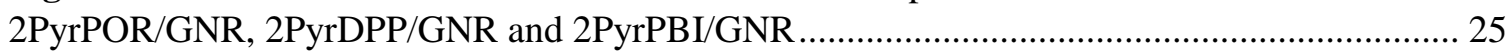

Figure S25. Calculation of the \% of CT states populated from the initial energy transfer event . 27

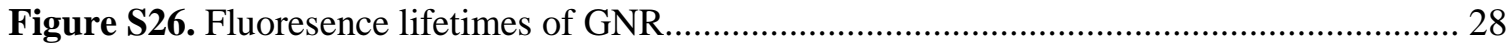

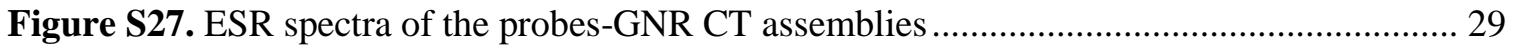

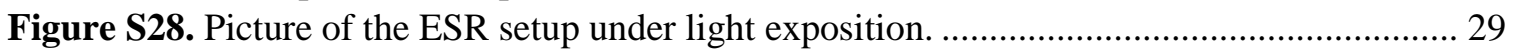

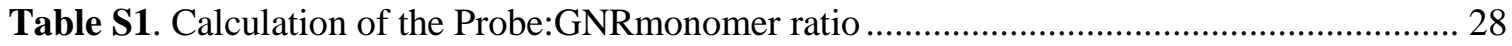

\section{Characterization}

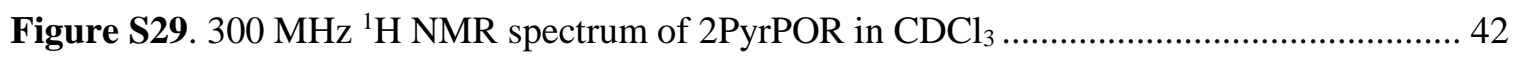

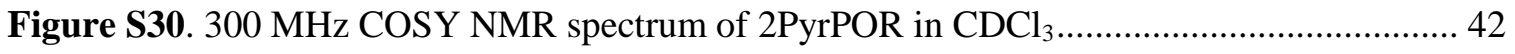

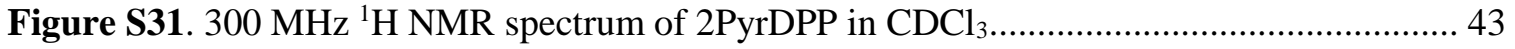

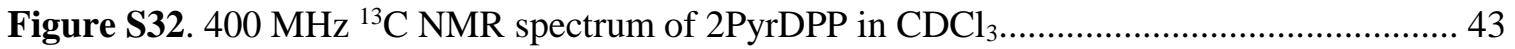

Figure S33. High resolution mass spectrum (APCI+) of 2PyrDPP ........................................... 44 


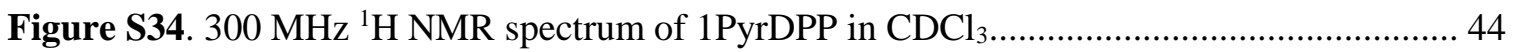

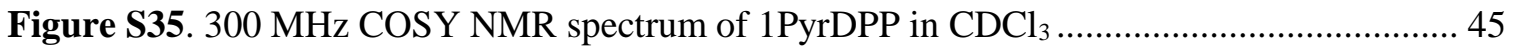

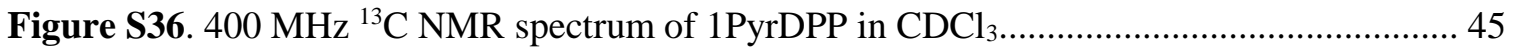

Figure S37. High resolution mass spectrum (ESI+) of 1PrDPP .................................................. 46

Figure S38. $300 \mathrm{MHz}{ }^{1} \mathrm{H}$ NMR spectrum of $2 \mathrm{PyrPBI}$ in $\mathrm{CDCl}_{3}$ (note: the recrystallisation of

2PyrPBI inside the NMR tube decreases the 2PyrPBI signal and overexpresses the residual

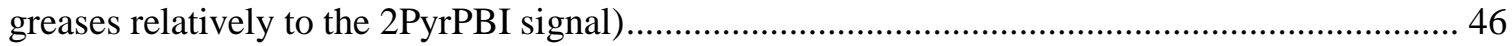

Figure S39. High resolution mass spectrum (APCI+) of 2PyrPBI ............................................ 47

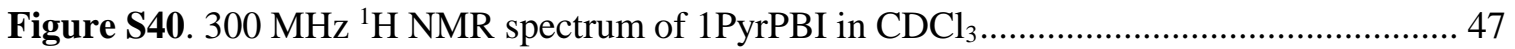

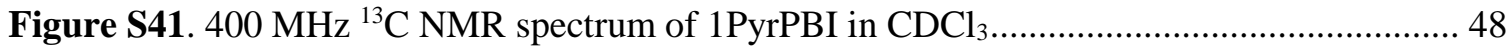

Figure S42. High resolution mass spectrum (APCI+) of 1PyrPBI ........................................ 48

Figure S43. $400 \mathrm{MHz}{ }^{1} \mathrm{H}$ NMR spectrum of boron, difluoro[3-iodo-5-[1-(4-iodo-3,5-dimethyl-

2H-pyrrol-2-ylidene- $\mathrm{N}$ )hexadecyl]-2,4-dimethyl-1H-pyrrolato- $\mathrm{NN}]$ in $\mathrm{CDCl}_{3} \ldots \ldots \ldots \ldots \ldots \ldots \ldots \ldots . . . . . . . . .49$

Figure S44. $400 \mathrm{MHz}{ }^{13} \mathrm{C}$ NMR spectrum of boron, difluoro[3-iodo-5-[1-(4-iodo-3,5-dimethyl-

2H-pyrrol-2-ylidene- $\mathrm{NN}$ )hexadecyl]-2,4-dimethyl-1H-pyrrolato- $\mathrm{NN}]$ in $\mathrm{CDCl}_{3} \ldots \ldots \ldots \ldots \ldots \ldots \ldots . . . . . . . . . .49$

Figure S45. High resolution mass spectrum (ESI+) of boron, difluoro[3-iodo-5-[1-(4-iodo-3,5-

dimethyl-2H-pyrrol-2-ylidene- $\mathrm{N}$ )hexadecyl]-2,4-dimethyl-1H-pyrrolato- $\mathrm{NN}]$........................... 50

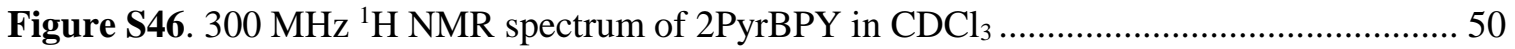

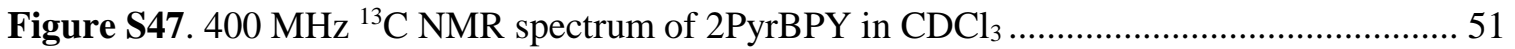

Figure S48. High resolution mass spectrum (ESI+) of 2PyrBPY ............................................ 51

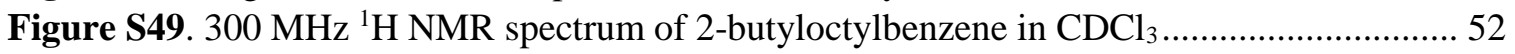

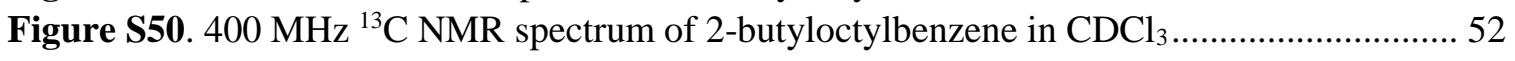

Figure S51. High resolution mass spectrum (APCI+) of 2-butyloctylbenzene ............................53

Figure S52. $300 \mathrm{MHz}{ }^{1} \mathrm{H}$ NMR spectrum of 1-(bromomethyl)-4-(2-butyloctyl)benzene in $\mathrm{CDCl}_{3}$

Figure S53. $400 \mathrm{MHz}{ }^{13} \mathrm{C}$ NMR spectrum of 1-(bromomethyl)-4-(2-butyloctyl)benzene in $\mathrm{CDCl}_{3}$

Figure S54. High resolution mass spectrum (ESI+) of 1-(bromomethyl)-4-(2-butyloctyl)benzene

Figure S55. $300 \mathrm{MHz}{ }^{1} \mathrm{H}$ NMR spectrum of 1,3-bis(4-(2-butyloctyl))propan-2-one in $\mathrm{CDCl}_{3} \ldots 55$ Figure S56. $300 \mathrm{MHz}{ }^{13} \mathrm{C}$ NMR spectrum of 1,3-bis(4-(2-butyloctyl))propan-2-one in $\mathrm{CDCl}_{3}$.. 55 Figure S57. High resolution mass spectrum (ESI+) of 1,3-bis(4-(2-butyloctyl))propan-2-one ... 56 Figure S58. $300 \mathrm{MHz}{ }^{1} \mathrm{H}$ NMR spectrum of 3-(3-bromophenyl)-2,5-bis(4-(2-butyloctyl)phenyl)-

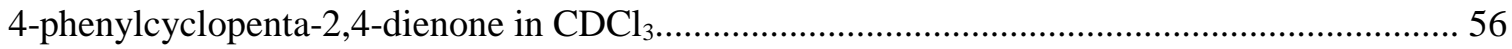

Figure S59. $300 \mathrm{MHz}{ }^{13} \mathrm{C}$ NMR spectrum of 3-(3-bromophenyl)-2,5-bis(4-(2-butyloctyl)phenyl)-

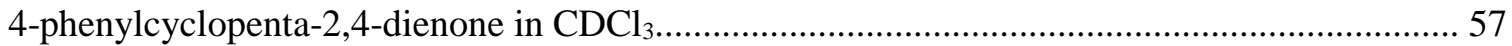

Figure S60. High resolution mass spectrum (ESI+) of 3-(3-bromophenyl)-2,5-bis(4-(2butyloctyl)phenyl)-4-phenylcyclopenta-2,4-dienone

Figure S61. $300 \mathrm{MHz}{ }^{1} \mathrm{H}$ NMR spectrum of 3-(3-ethynylphenyl)-2,5-bis(4-(2-butyloctyl)phenyl)4-phenylcyclopenta-2,4-dienone in $\mathrm{CDCl}_{3}$ 58

Figure S62. $300 \mathrm{MHz}{ }^{13} \mathrm{C}$ NMR spectrum of 3-(3-ethynylphenyl)-2,5-bis(4-(2butyloctyl)phenyl)-4-phenylcyclopenta-2,4-dienone in $\mathrm{CDCl}_{3}$ 58

Figure S63. High resolution mass spectrum (ESI+) of 3-(3-ethynylphenyl)-2,5-bis(4-(2butyloctyl)phenyl)-4-phenylcyclopenta-2,4-dienone 59

Figure S64. $300 \mathrm{MHz}{ }^{1} \mathrm{H}$ NMR spectrum of GNR precursor in $\mathrm{CDCl}_{3}$ 59 


\section{TEM images and GPC traces}
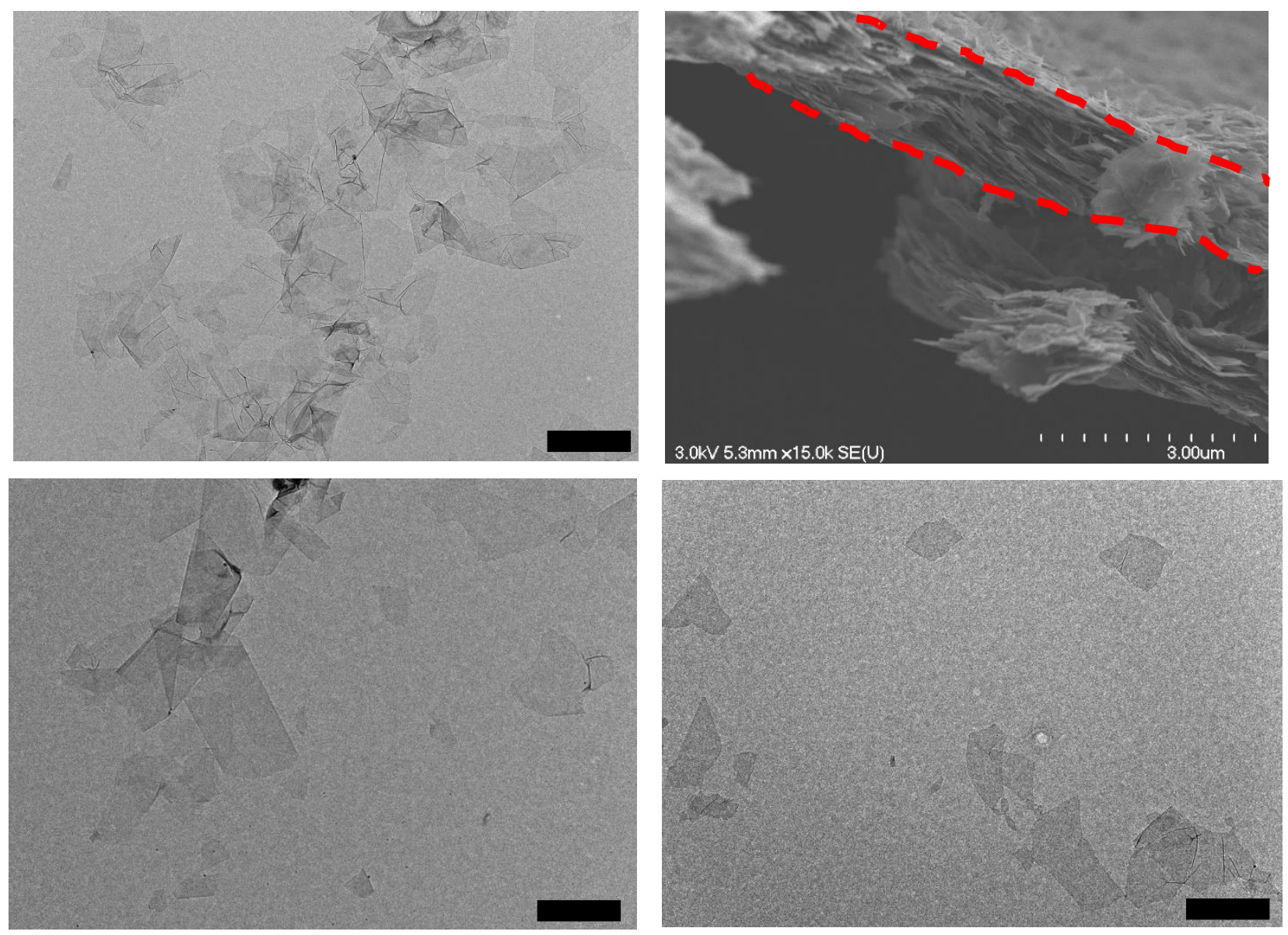

Figure S1. (a-c) TEM images of RGO dispersed in ethanol, sonicated and deposited on an electron microscopy copper grid and (d) SEM image of RGO films obtained by filtration of an RGO-containing solution on a $0.2 \mu \mathrm{m}$ Anodisc inorganic filter membrane and transferred onto a copper disc. 


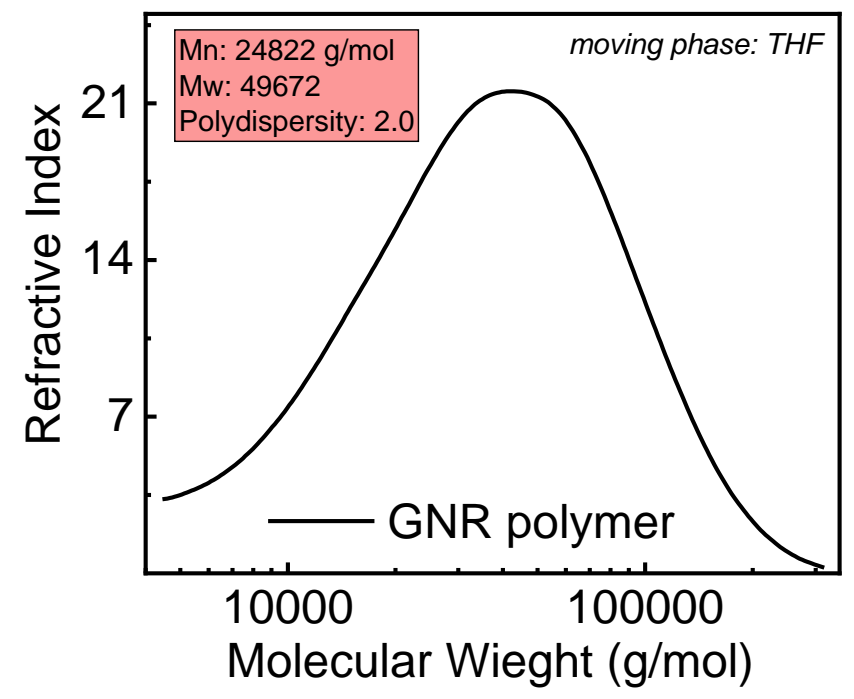

Figure S2. GPC trace of the GNR polymer in THF. Degree of polymerization $=15$ units $\left(M_{n}\right)$ or 69 units $\left(M_{w}\right)$

\section{Adsorption of probes on RGO}

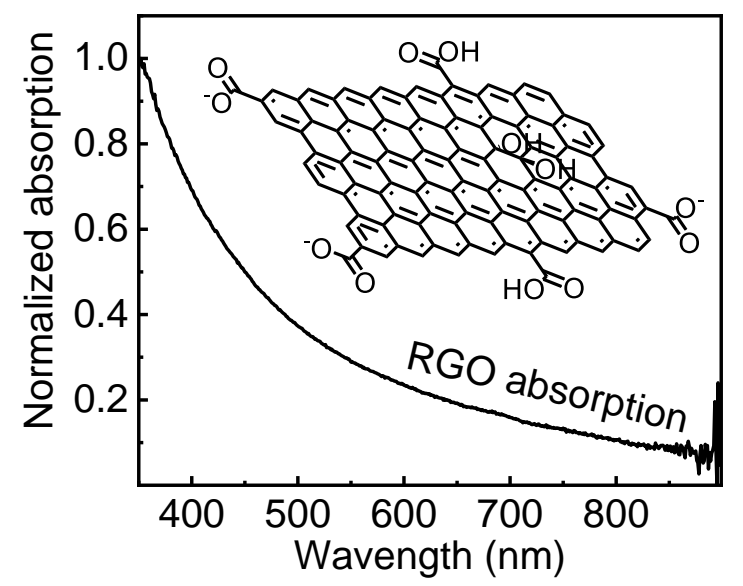

Figure S3. Normalized absorption spectrum of $\mathrm{RGO}$ dispersed in $\mathrm{H}_{2} \mathrm{O}$. The original absorbance of the sample was below 1. 

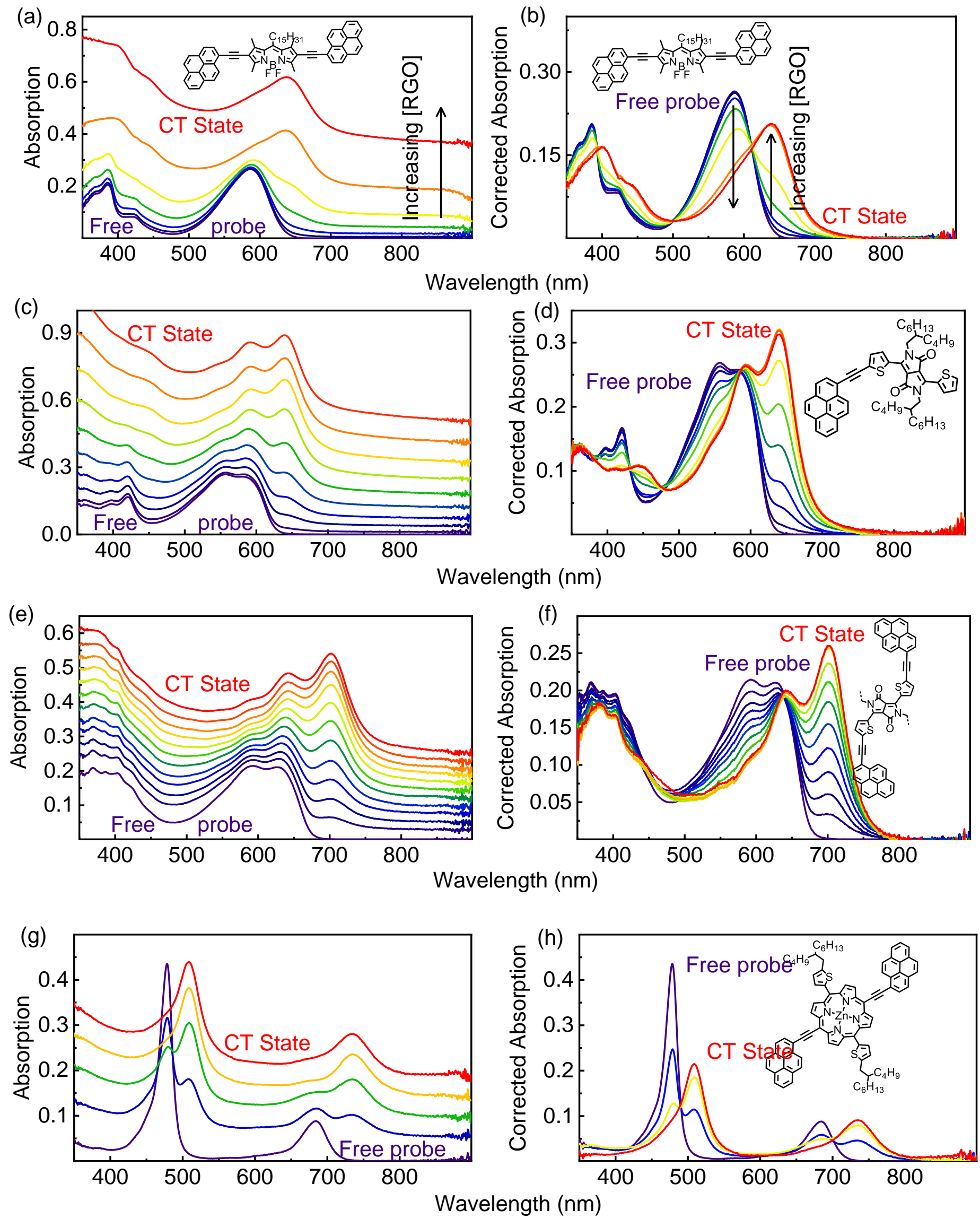

Page S-6 

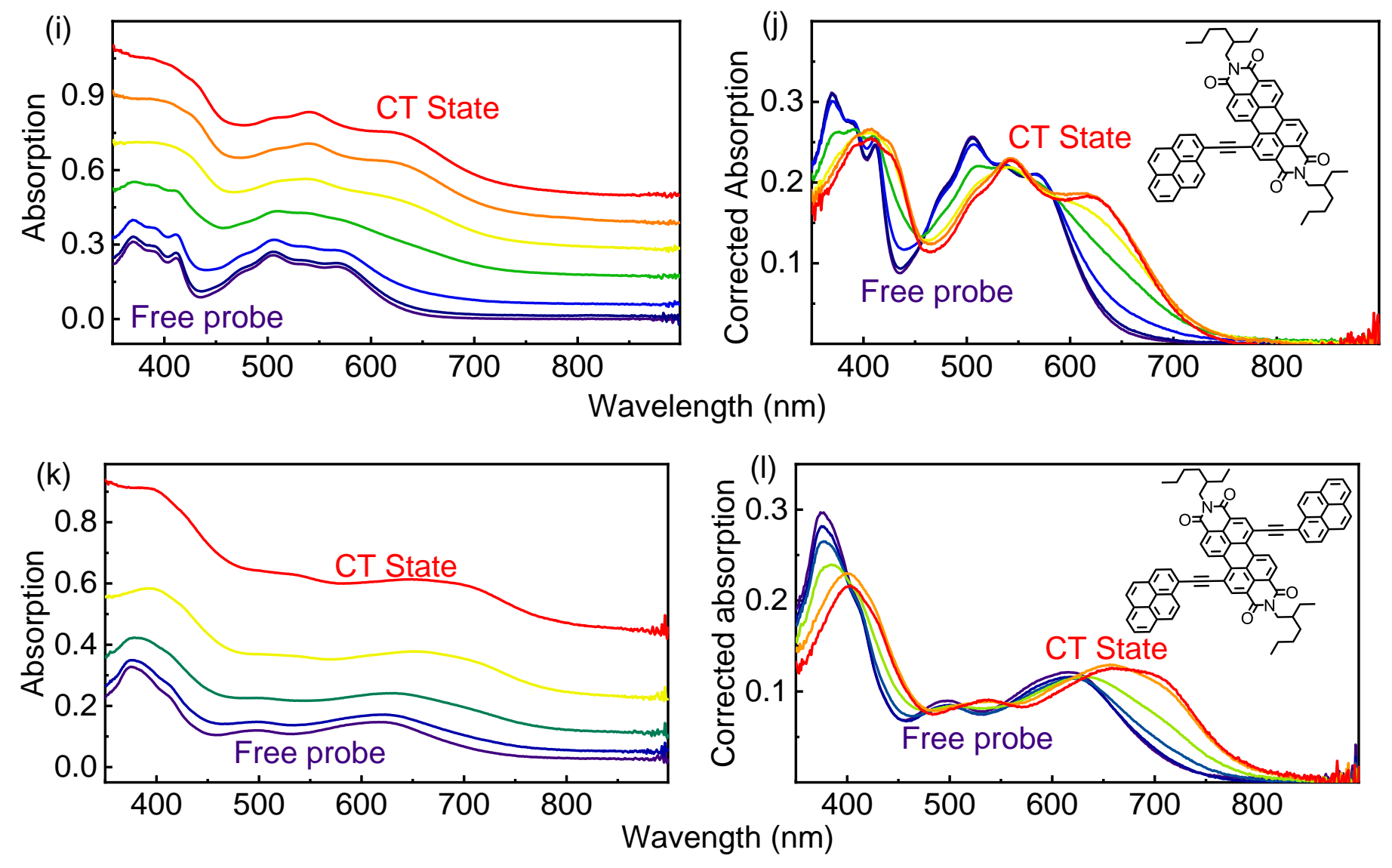

Figure S4. Evolution of the absorption spectra of $2 \operatorname{PyrBPY}(a, b), 1 \operatorname{PyrDPP}(c, d)$, 2PyrDPP (e, f), 2PyrPOR (g, h), 1PyrPBI $(i, j)$ and 2PyrPBI $(k, 1)$ upon increasing the RGO content from free probe (purple) to fully adsorbed probe (red) in DMF. Uncorrected (left) and corrected absorptions from GNR contribution (corrected absorption $=\mathrm{Abs}(\mathrm{RGO}+$ Probe)-Abs(RGO); (right)). 

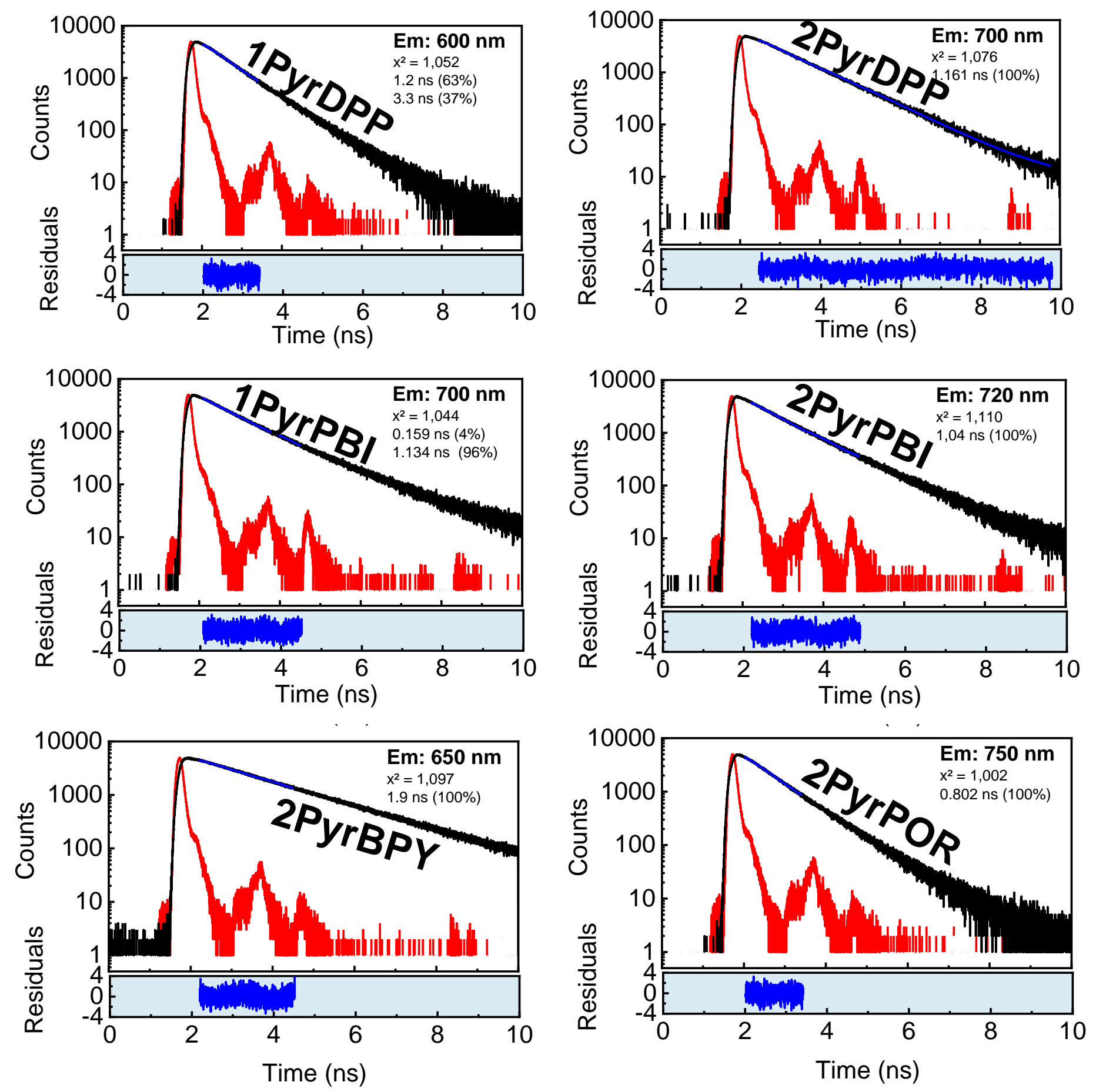

Figure S5. Fluorescence decay traces (black traces) and their lifetimes of the free dyes in $\mathrm{CHCl}_{3}$ at $273 \mathrm{~K}\left(\lambda_{\text {exc }}=477 \mathrm{~nm}\right)$. Red trace $=$ pulse profile. Blue trace $=$ residual. The solutions were degassed by bubbling $\operatorname{Ar}(\mathrm{g})$ for $5 \mathrm{~min}$. 


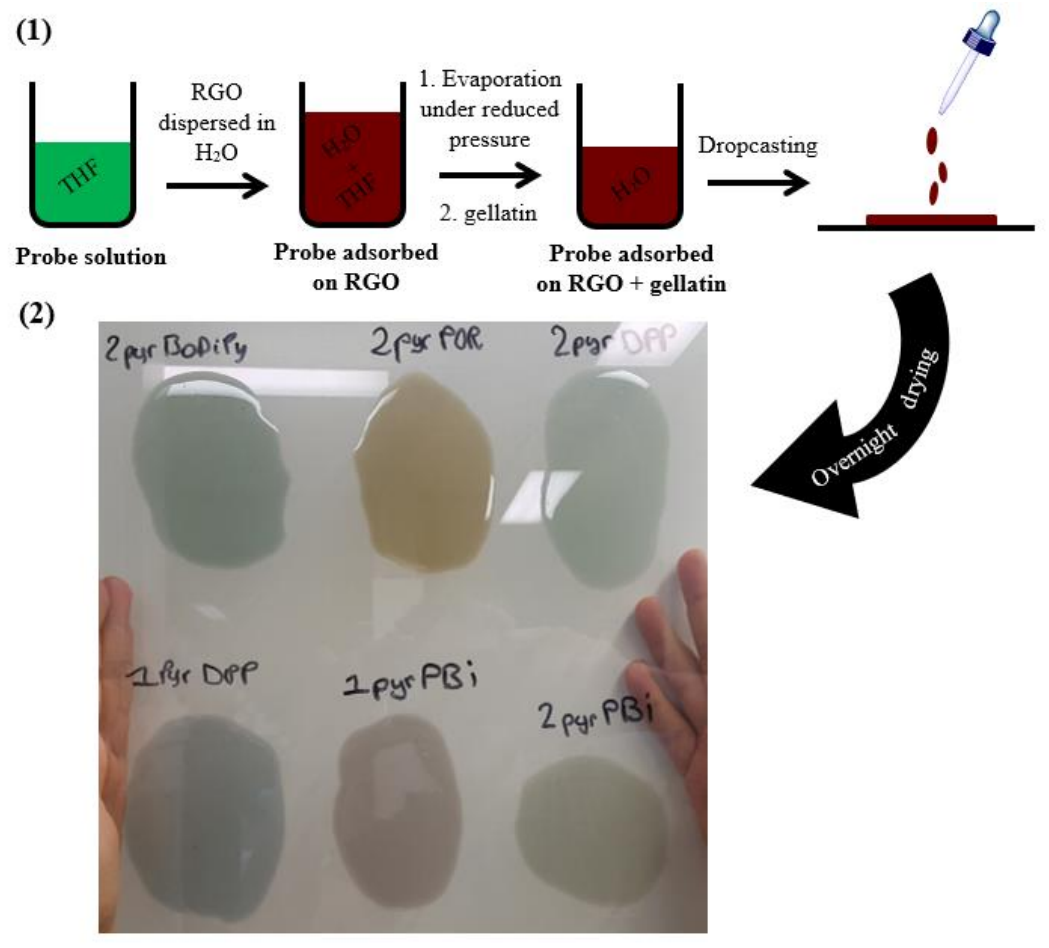

Figure S6. (1) Cartoon showing the method used to prepare the suspensions of dyes adsorbed on RGO in gelatin. (2) Picture of the resulting films.

\section{Adsorption of probes on GNR}

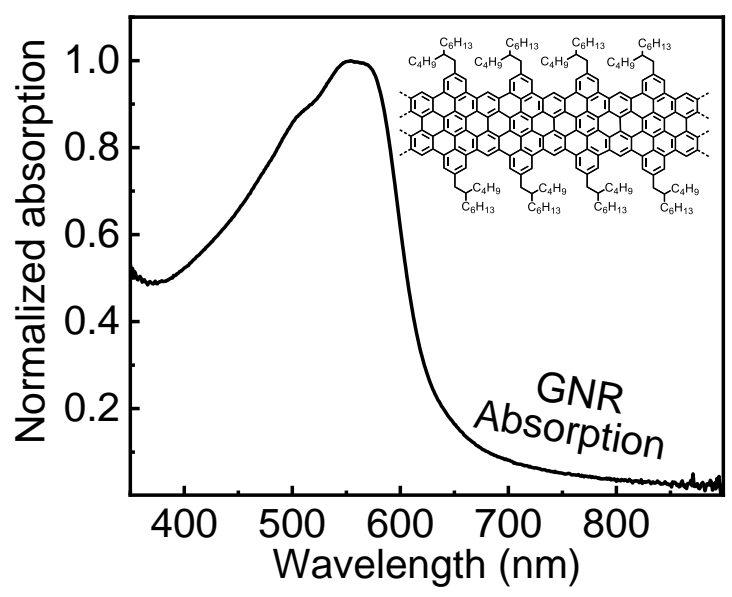

Figure S7. Normalized absorption spectrum of $\mathrm{GNR}$ in $\mathrm{CHCl}_{3}$. The original absorbance of the sample was below 1 . 

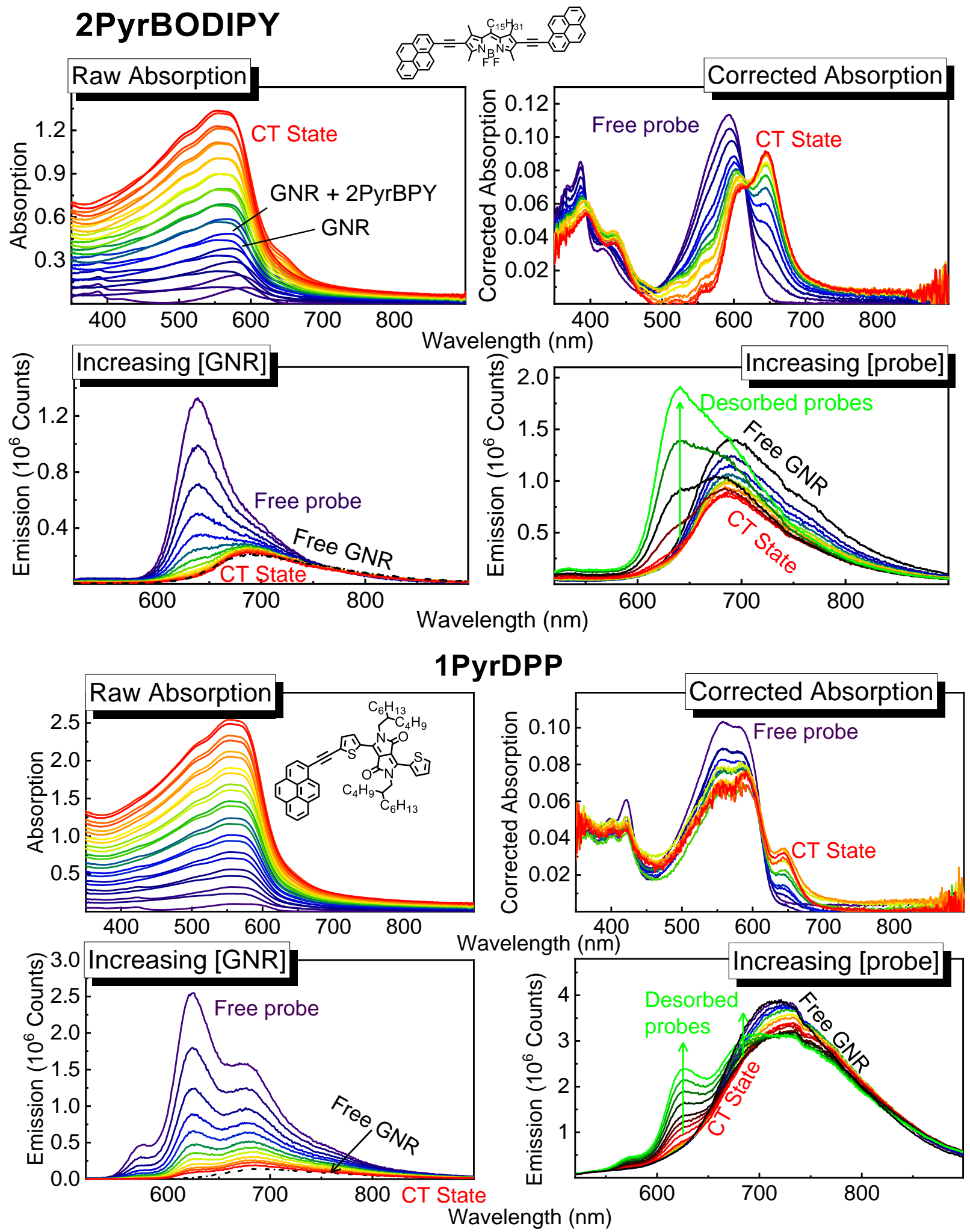

Figure S8. Evolution of the absorption spectra of the probes for Case 1 (1PyrDPP and 2PyrBPY) upon increasing the GNR content from free dyes (purple) to fully adsorbed dyes (red) in $\mathrm{CHCl}_{3}$. Uncorrected (top left), corrected absorption spectra (top right) and evolution of their corresponding fluorescence spectra (bottom left). 

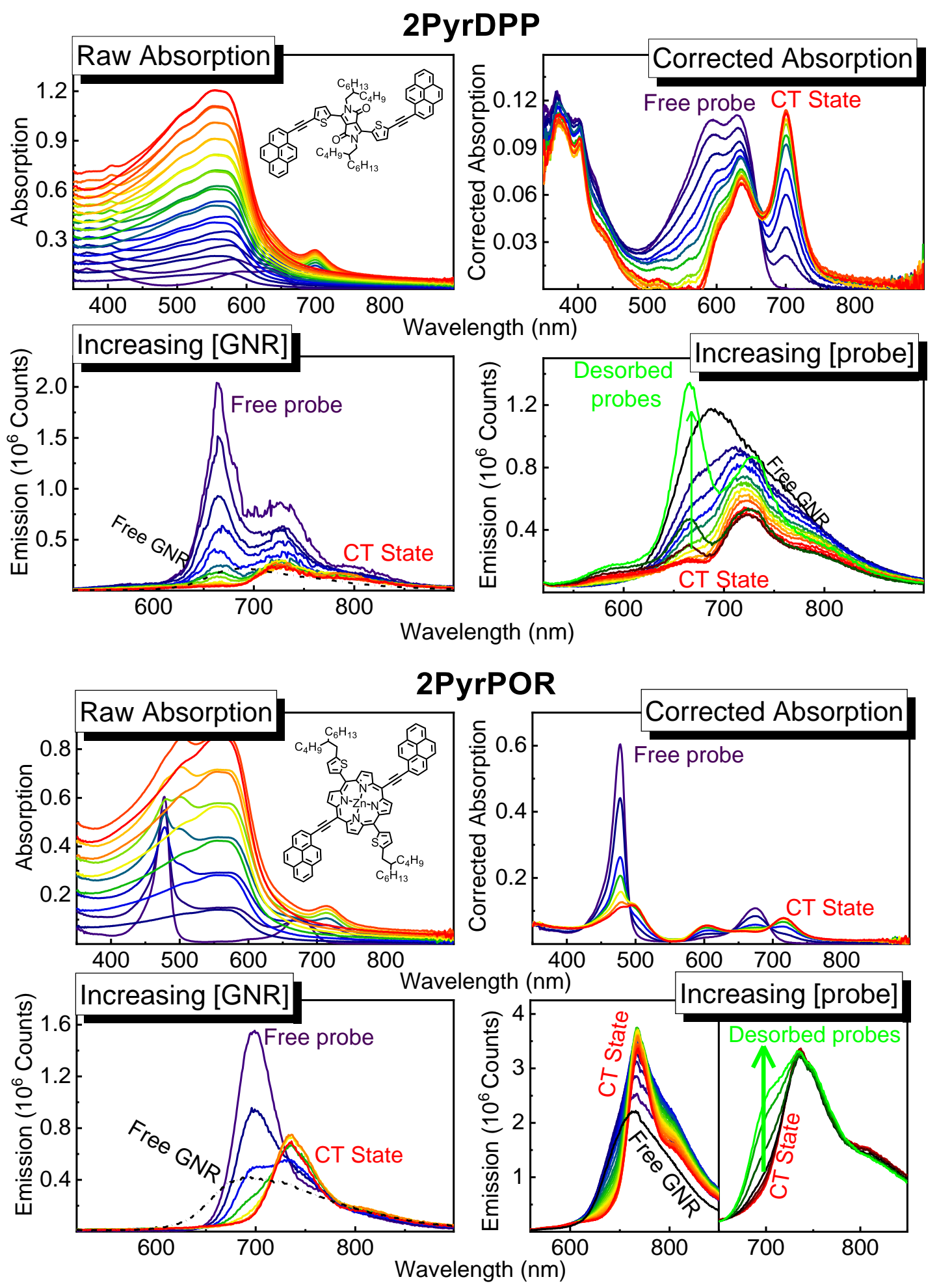

Figure S9. Evolution of the absorption spectra of the probes for Case 2 (2PyrPOR and 2PyrDPP) upon increasing the GNR content from free dyes (purple) to fully adsorbed dyes (red) in $\mathrm{CHCl}_{3}$. Uncorrected (top left), corrected absorption spectra (top right) and evolution of their corresponding fluorescence spectra (bottom left). 

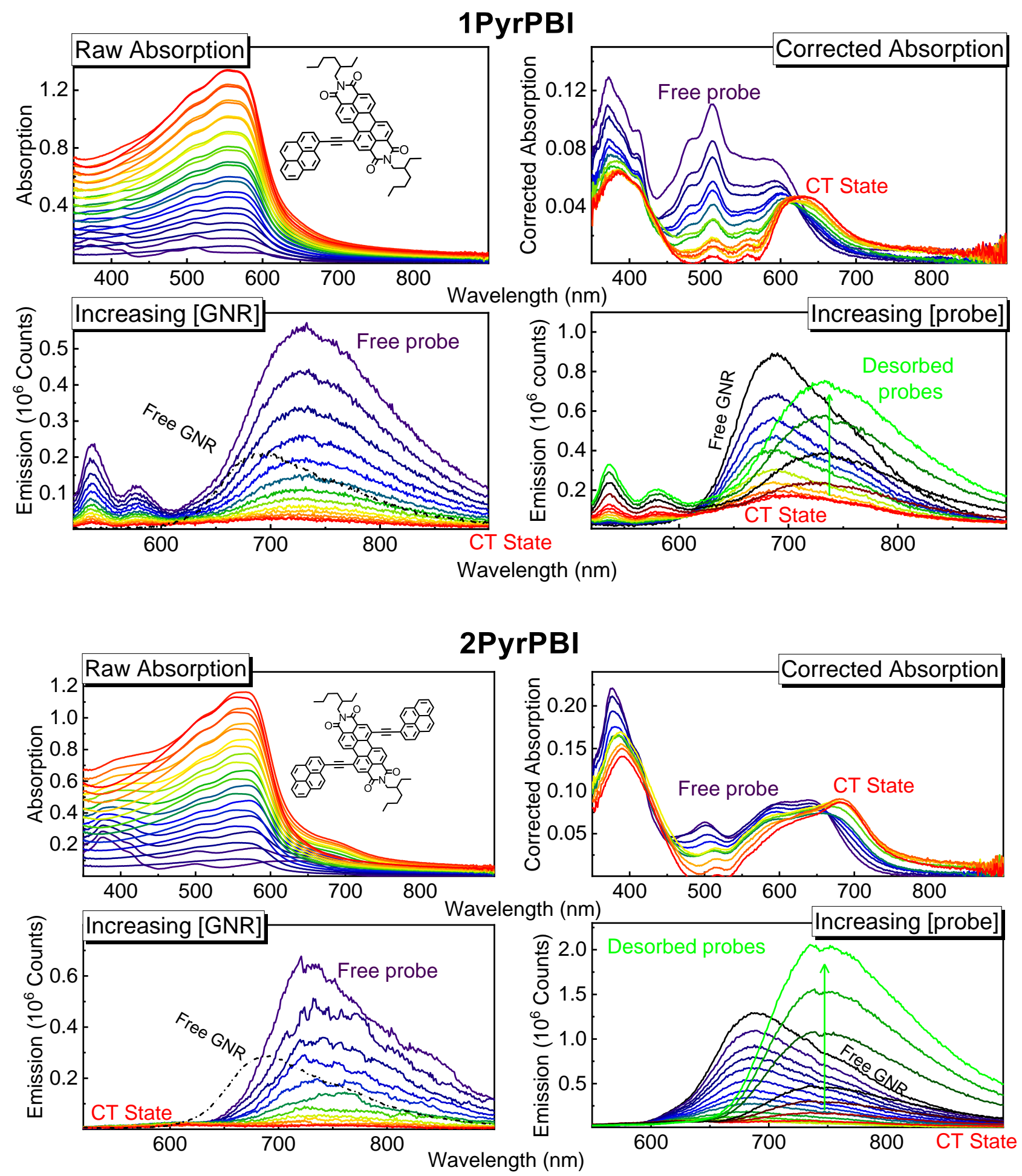

Figure S10. Evolution of the absorption spectra of the probes for Case 3 (2PyrPBI and 1 PyrPBI) upon increasing the GNR content from free dyes (purple) to fully adsorbed dyes (red) in $\mathrm{CHCl}_{3}$. Uncorrected (top left), corrected absorption spectra (top right) and evolution of their corresponding fluorescence spectra (bottom left). 


\section{Are all probes fully adsorbed on the GNR?}

A key point is that a very minimal quantity of free probes remains in solution prior to analyses. Three experimental evidences are provided:

1) The shift of the absorption bands. The two main absorption bands are at 594 and 629 $\mathrm{nm}$ for the free 2PyrDPP and at 638 and $700 \mathrm{~nm}$ for the adsorbed 2PyrDPP. The $9 \mathrm{~nm}$ difference between the lower absorption band of free 2PyrDPP and the higher absorption one of the adsorbed 2PyrDPP is large enough for a good analysis for assessing the absence of free probes. The TAS measurements (last section of the text and Figure 10b) also supports that the 638 and $700 \mathrm{~nm}$ peaks are two vibronic features of a same CT complex.

2) The decrease of the absorption band of free 2PyrDPP. The $594 \mathrm{~nm}$ absorption band of free 2PyrDPP experiences an $84 \%$ decrease in intensity suggesting that at least $84 \%$ of all probes have been converted into CT complexes. Concurrently, the emission band undergoes a $\sim 98 \%$ reduction in intensity. These two observations are good indicators that most 2PyrDPP probes are fully adsorbed.

3) The saturation limitation. When increasing the GNR content, the free probes (purple line) is progressively converted to the CT complexes (red line). The spectral changes from free to adsorbed probes are first rapid (purple to green). Then, the progression slows down progressing towards a saturation limit (green to orange) until no more spectral changes occur (orange to red). At point the free $\leftrightarrow$ adsorbed probes equilibrium is reached, which is optimally shifted toward the adsorbed species.

Noteworthy, while it cannot be completely discarded that some residual free probes are present in solution, they only weakly participate to the total absorption. Consequently, the reported spectra in this work are reasonably assumed free of free probe absorption.

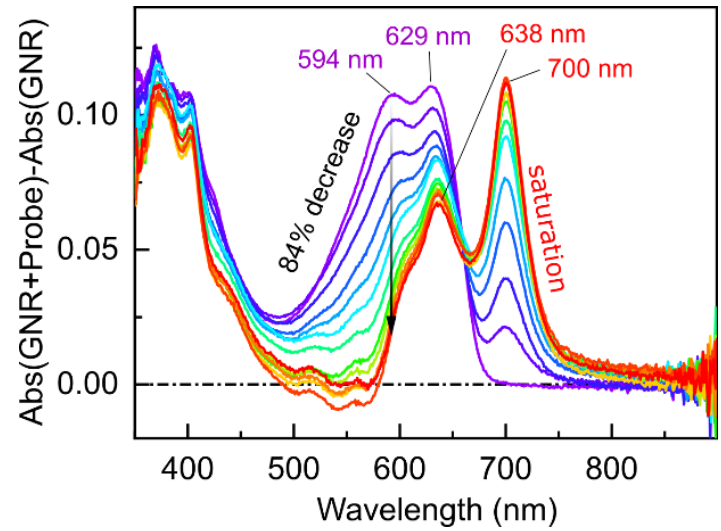

Figure S11. Evolution of the absorption spectrum of 2PyrDPP upon increasing the GNR content going from only free 2PyrDPP (violet) in solution to adsorbed 2PyrDPP (red line) at full GNR saturation. 


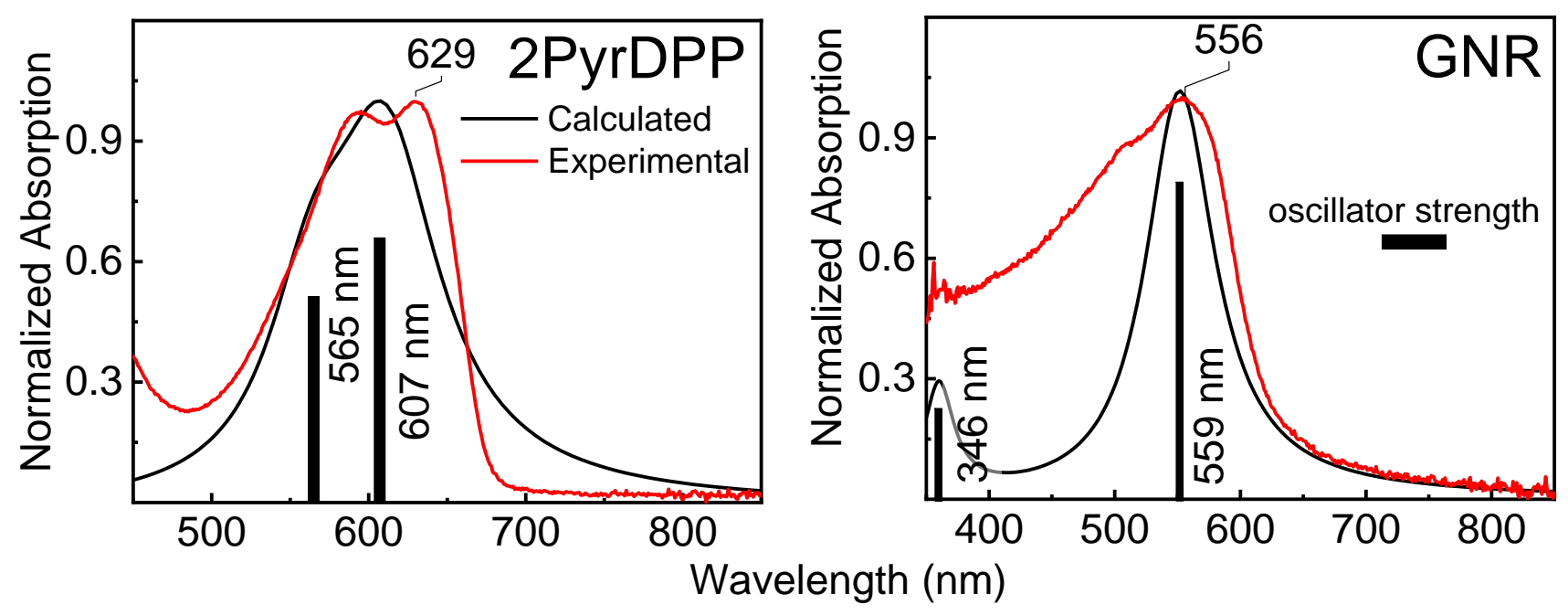

Figure S12. Bar graph reporting the calculated positions and oscillation strengths (black bar) of the electronic transitions calculated by TDDFT, the normalized simulated spectrum (black line) in gas phase with the normalized absorption spectra of GNR and 2PyrDPP in $\mathrm{CHCl}_{3}$ (red lines). The theoretical spectrum is generated with an arbitrary thickness of 0.01 Rydberg $=1097 \mathrm{~cm}^{-1}$ for each bar.

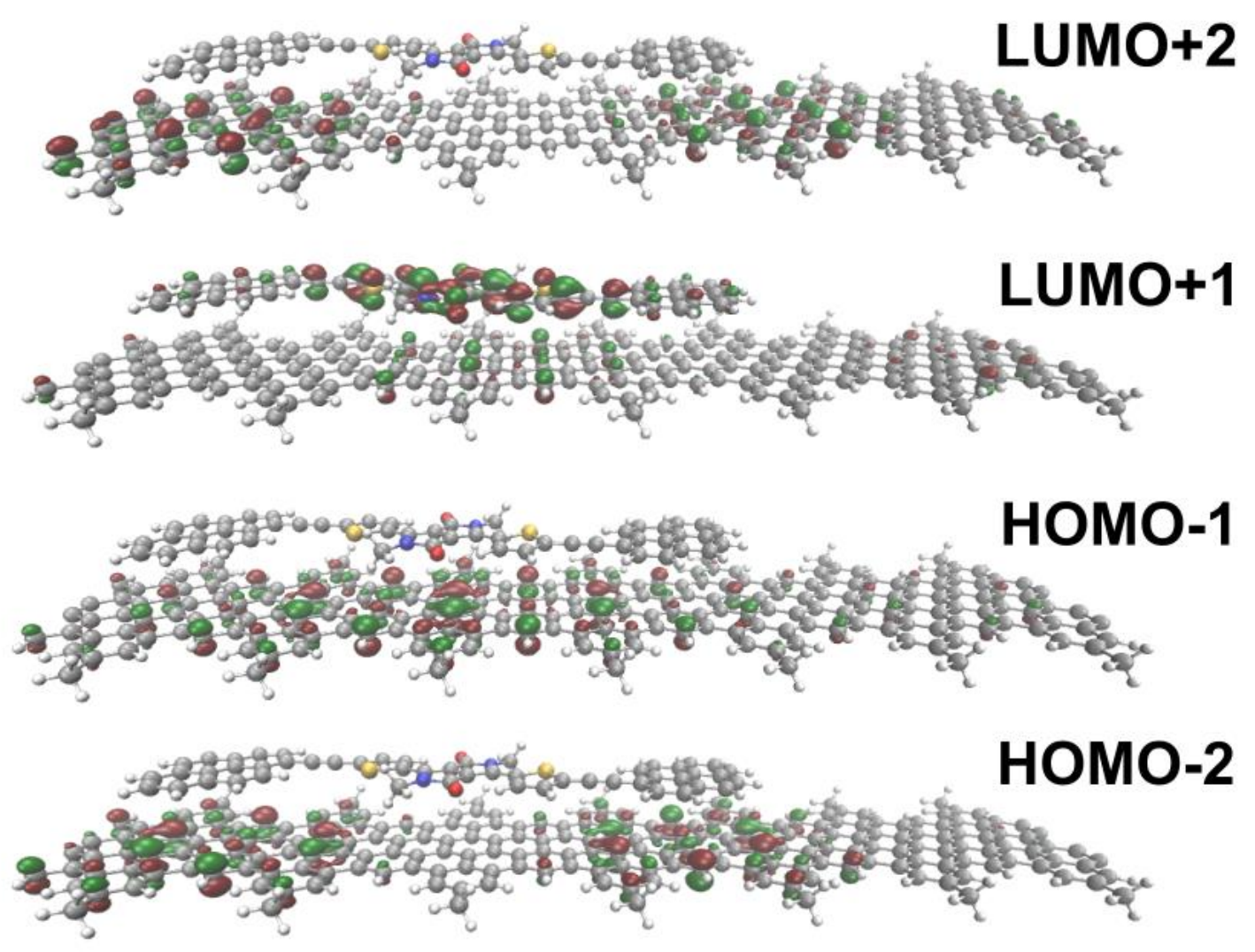

Figure S13. Representations of the HOMO-2, HOMO-1, LUMO+1 and LUMO+2 for the 2PyrDPP-GNR assembly. 
Equilibrium between adsorbed and free probes. To shed light on the origin of the adsorption, these processes were investigated at various concentrations. Figure S14a exhibits the evolution of the absorption spectra of a GNR-containing solution upon increasing the 2PyrDPP concentration. At low concentrations, where GNR is in large excess ( 1:120 2PyrDPP:GNR monomer ), the spectra exhibit the characteristic band at 701 $\mathrm{nm}$ (purple line). Upon increasing the 2PyrDPP concentration further, the UV-vis spectra evolve, and the signal associated with the free probe appears. This change is accompanied by the appearance of the dye fluorescence. At the end of these additions, the absorption spectra exhibit the complete spectral signature of free 2PyrDPP (red line).

The monitoring of the absorbance bands of the low-energy CT assembly upon adding dyes, which translates into quantifying the amount of formed CT assemblies, exhibits a linear behavior at low concentrations (Figure S14b). In this concentration range, the dye is almost totally adsorbed, but at higher concentrations, the spectral contribution of the CT assembly band starts to damp. Indeed, at higher concentrations the spectral contribution of the CT assembly bands starts to saturate with a maximum for a 2 PyrDPP:GNR monomer $_{\text {ratio }}$ approaching $\approx 1: 10$, which corresponds to $\sim 35 \%$ of the total GNR polymer surface filled (see - - - line in Figure S14b). First, this study indicates that not all the GNR surface is permanently and fully filled. Second, the absence of complete adsorption (only $35 \%$ ) may stem from inter-GNR chain aggregation in solution. The concentration dependence of the adsorption spectra indicates that the presence of a thermodynamic association/dissociation process where the equilibrium of assembled dyes $v s$. free dye favors the adsorbed state at low concentrations. Thus, these experiments further confirm that the adsorbed probes are the main species interacting with the GNRs.

This study was extended to all dyes but a clear spectral distinction of the free dyes from assembled ones was not always possible, especially for $2 \mathrm{PyrPBI}$ and 1 PyrPBI. Due to the lack of vibrational features, the evaluation of the free dye/assembled CT complex ratio prevented accuracy in the deconvolution process. However, the relative assembly strength (i.e. the maximum surface filling) of the remainder dyes was easily assessed. 2PyrDPP is found to be the strongest interacting dye, followed by 2PyrPOR, 2PyrBPY and 1PyrDPP reaching a maximum of approximately $\approx 27-23 \%$ of surface filling. These results also stress the relationship between solubility and aggregation strength as previously mentioned. Indeed, both 2PyrPBI and 2PyrPOR exhibit the largest surface coverage but strikingly, they are also the least soluble probes, thus driving them to quasi-irreversible assembly formation. The weakest interacting dyes are 1PyrPBI, 2PyrBPY and 1PyrDPP with 14, 12 and $9 \%$ surface coverage, respectively. These results are consistent with the fact that 1 PyrDPP is still showing traces of emission arising from free 1PyrDPP even near full GNR saturation. 

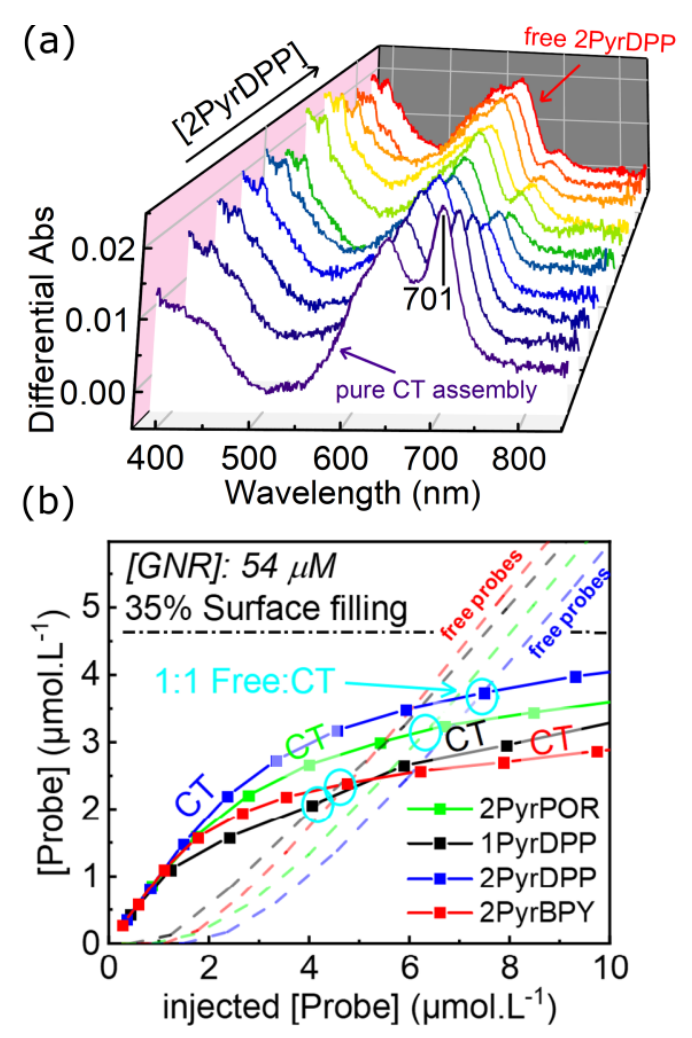

Figure S14. (a) Evolution of the 2PyrDPP absorption spectra upon increasing its concentration in a GNR-containing solution $[\mathrm{GNR}]=54 \mu \mathrm{M}$; note that the absorption spectra have been corrected for the GNR absorption signal. (b) Evolution of the generated adsorbed probes on GNR concentration with increasing concentration of the added probe in a $54 \mu \mathrm{M}$ GNR solution. The broken line represents the concentration of free probes in solution. 


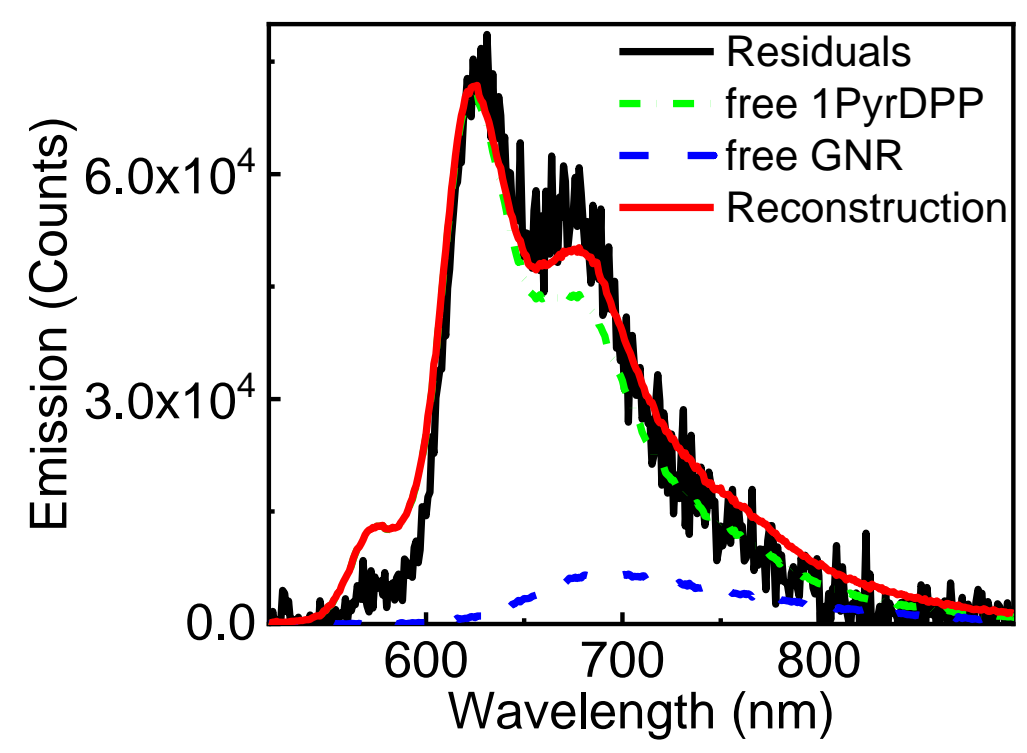

Figure S15. Deconvolution of the residual emission (black) of 1PyrDPP (i.e. emission of 1PyrDPP adsorbed on GNR minus the emission of a solution of equivalent concentration of pristine GNR). The free 1PyrDPP emission contribution is in green and the free GNR emission contribution is blue. The linear combination of these two contributions (red) overlaps most of the residual emission. 


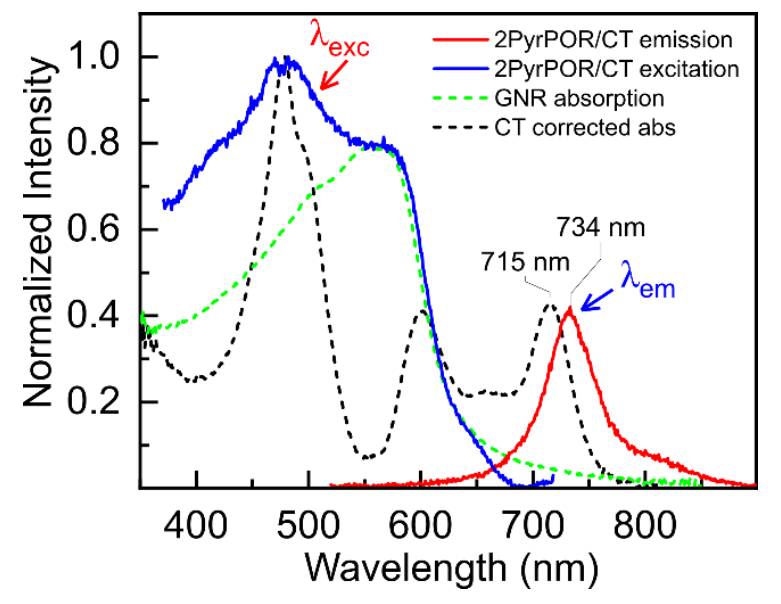

Figure S16. Normalized GNR absorption (green line), corrected 2PyrPOR CT complex absorption (black line), excitation ( $\lambda_{\mathrm{em}}=740 \mathrm{~nm}$; blue line) and emission $\left(\lambda_{\mathrm{exc}}=500 \mathrm{~nm}\right.$; red line) spectra of the 2PyPOR/GNR CT assemblies in $\mathrm{CHCl}_{3}$. The relative proportions of probe-graphene are 1:36 2PyrPOR monomers vs 2PyrPOR/GNR assemblies. Note that the green and the red lines were normalized and adjusted respectively to the excitation and emission lines for clarity. 
(a)

Rayleigh

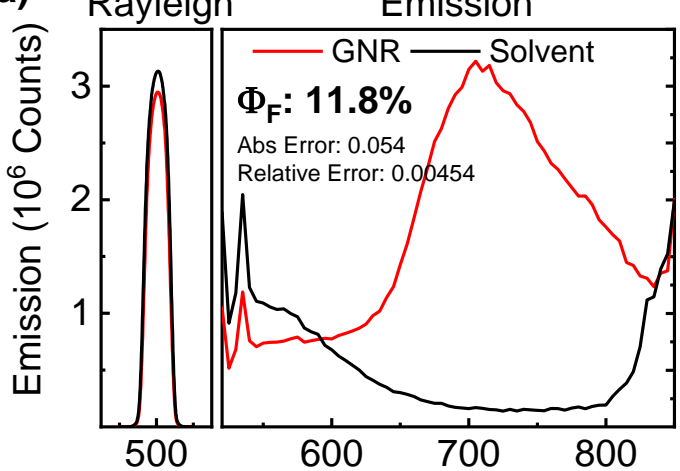

(c)

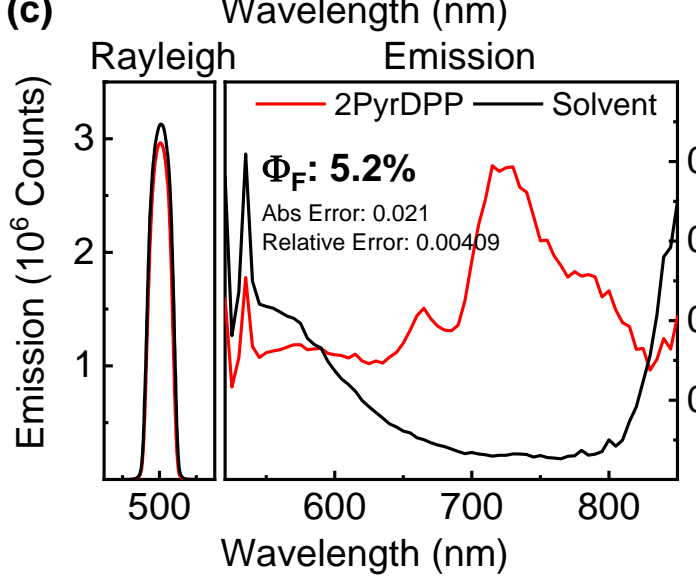

(b)
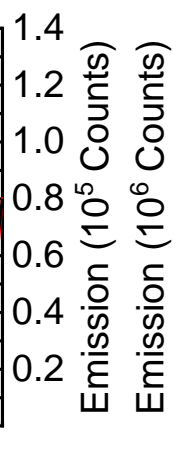

(d)
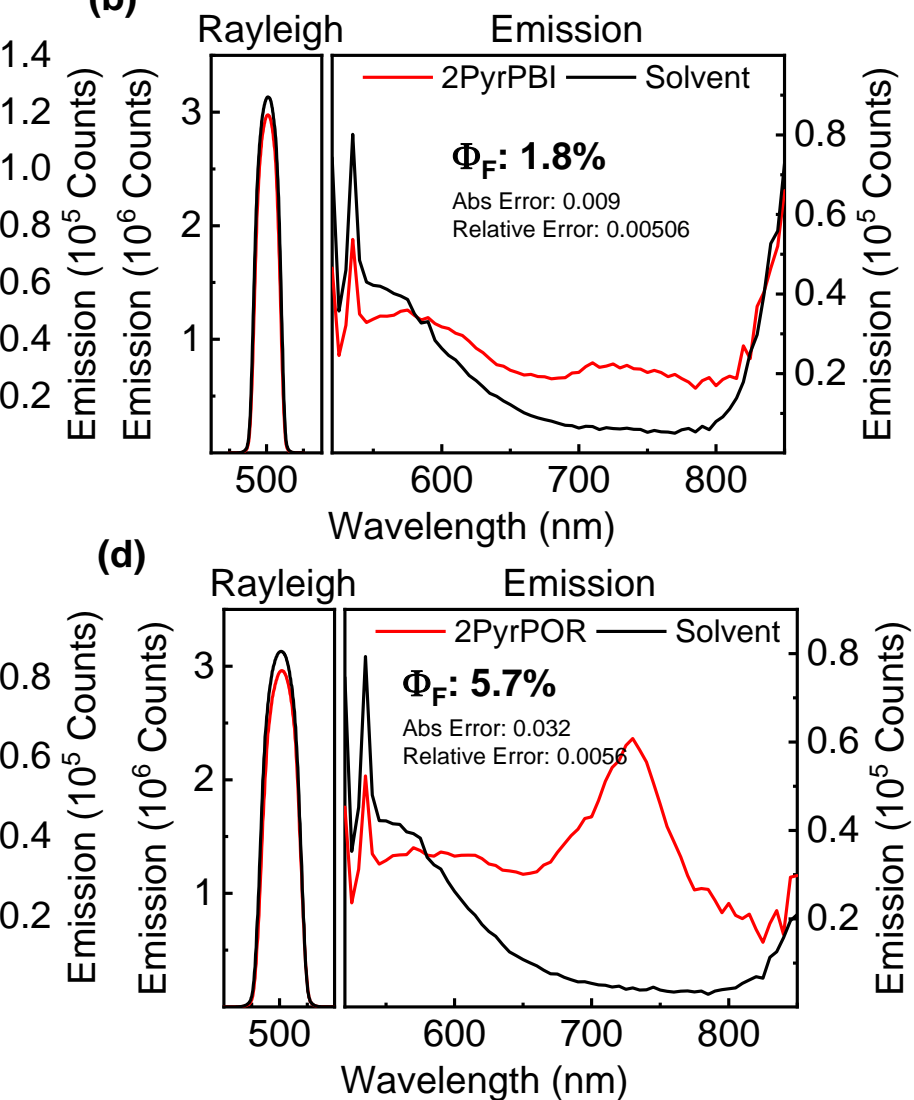

Figure S17. Fluorescence quantum yields (integration sphere), $\Phi_{\mathrm{F}}$, of pristine GNR (a), 2PyrPBI CT state (b), 2PyrDPP CT state (c) and 2PyrPOR CT state (d) in $\mathrm{CHCl}_{3}$ at $273 \mathrm{~K}$.

\section{Cyclic voltammetry of HOMOs}

All cyclic voltammetry (CV) measurements were performed using an Ag wire as reference electrode, a Pt mesh as counter electrode and a Pt electrode as working electrode. The couple ferrocene/ferrocenium was used as an internal reference. The CV measurements were performed in distilled acetonitrile and $99.9 \%$ pure spectroscopic grade $\mathrm{CHCl}_{3}$ respectively for GNR and probe measurements. All measurements were performed with an argon degassed $10^{-1} \mathrm{M}$ solution of tetrabutylammonium hexafluorophosphate. All probes CV measurements were made from a $10^{-3}$ $\mathrm{M}$ solution of probes in $\mathrm{CHCl}_{3}$. Due to the low solubility of $2 \mathrm{PyrPBI}, \mathrm{CHCl}_{3}$ was found to be the only solvent able to solubilize it and was thus used systematically for consistency. The CV measurement for GNR was performed in film. A solution of GNR in $\mathrm{CHCl}_{3}$ was drop casted on top of the Pt working electrode and the solvent was dried in the air. The process was repeated until GNR was fully covering the working electrode. Indeed, due to the high molecular mass of GNR, no clear oxidation or reduction wave was observed in liquid state. A typical phenomenon observed for conjugated polymers. The evaluation of the HOMO energies was performed as follow:

$$
E_{\text {Hомо }}(\mathrm{eV})=E_{a v}(V)-E_{F c^{+} / F c^{0}}(V)+4.8(\mathrm{eV})
$$

where $\mathrm{E}_{\mathrm{HOMO}}$ stands for the energy level of the $\mathrm{HOMO}, \mathrm{E}_{\mathrm{av}}$ for the average value between the anodic and cathodic electrical potential peaks of the first oxidation of the molecule and $4.8 \mathrm{eV}$ was used as the HOMO energy level of ferrocene. 
1PyrDPP

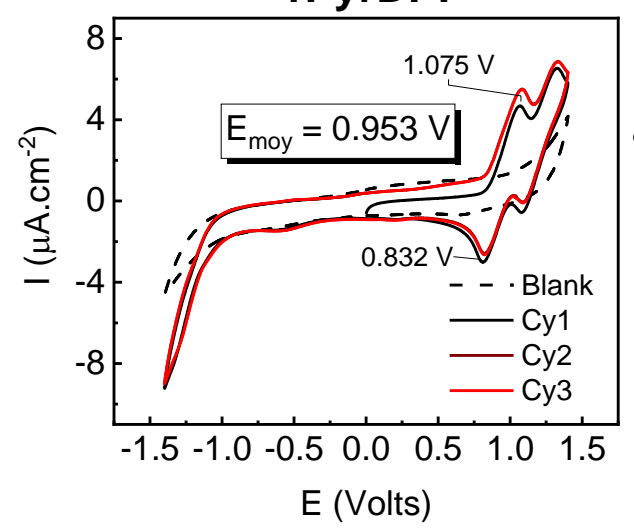

1PyrPBI

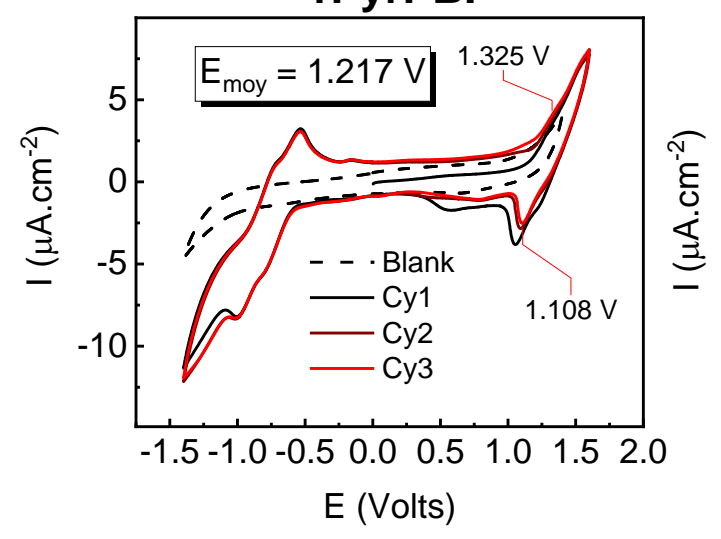

2PyrDPP

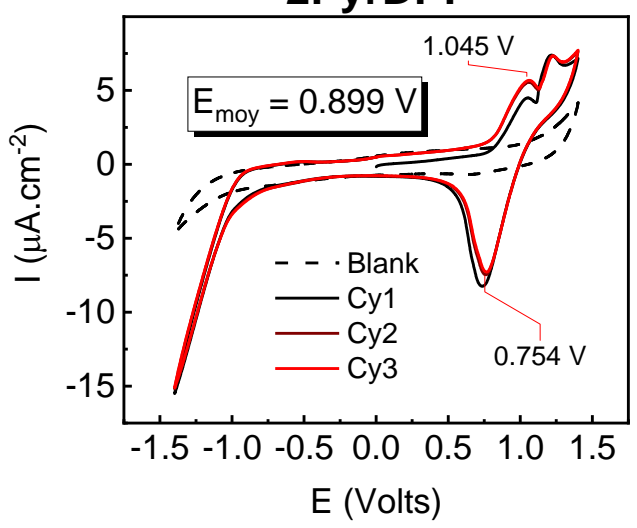

2PyrPBI

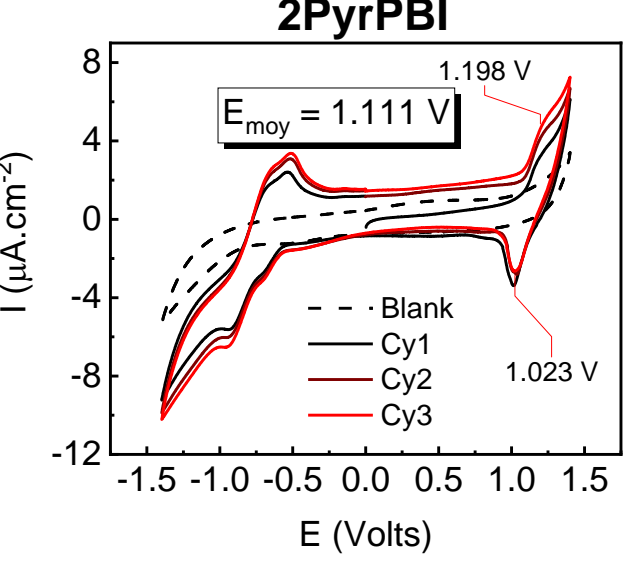

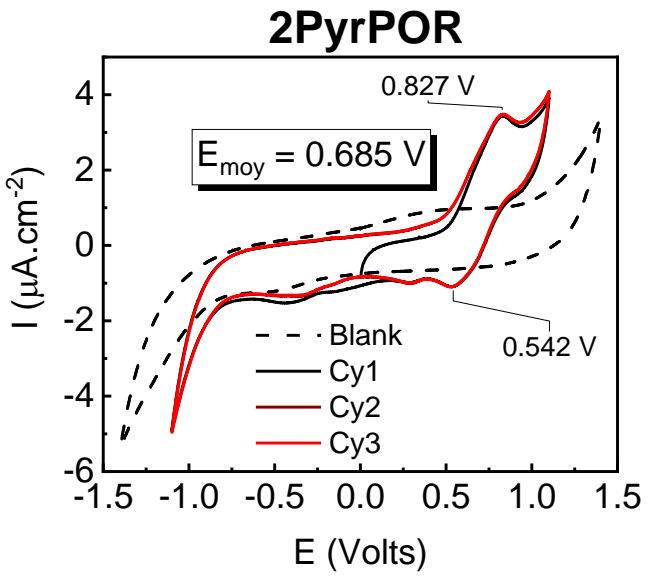

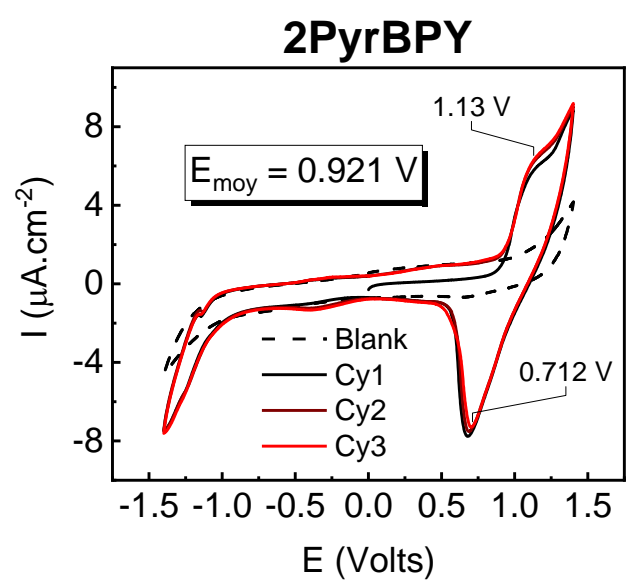

Figure S18. Cyclic voltammetry of the probes in $\mathrm{CHCl}_{3}$. The potential $\mathrm{E}(\mathrm{V})$ in the $\mathrm{x}$ axis is not corrected from $\mathrm{Fc} / \mathrm{Fc}^{+}$. Scan rate $=200 \mathrm{mV} / \mathrm{s}$. (- - is the pure $\mathrm{CHCl}_{3}$ cyclic voltammetry)

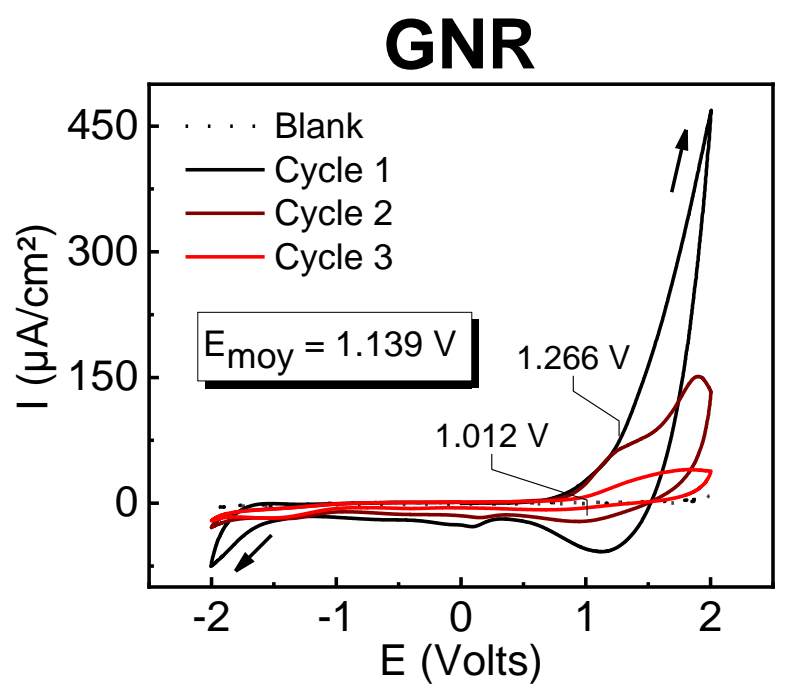

Figure S19. Cyclic voltammetry of GNR drop casted on top of the Pt electrode in acetonitrile. The potential $\mathrm{E}(\mathrm{V})$ on the $\mathrm{x}$ axis is not corrected from $\mathrm{Fc} / \mathrm{Fc}^{+}$. Scan rate $=200$ $\mathrm{mV} / \mathrm{s}$. 
Selectivity of the absorption of probes/GNR assemblies. Among the probe/GNR assemblies reported herein, 2PyrPOR, 2PyrDPP, and 2PyrPBI permitted selective excitation of either the uncomplexed GNR segment $\left(\lambda_{\text {exc }}=500 \mathrm{~nm}\right.$; except 550 $\mathrm{nm}$ for 2PyrPOR) or of the probes alone ( $\lambda_{\text {exc }}=700 \mathrm{~nm}$; except $720 \mathrm{~nm}$ for 2PyrPOR). Among all six probes, these three probes are the only one that exhibit bandgaps well below that those for GNR. Thus, allowing the excitation of the probes without exciting the GNR. The selectivity of the $500 \mathrm{~nm}$ excitation was first secured by the low surface filling of $10 \%$ of probes vs GNR polymers. The three samples used for TA experiments (colored lines, Figure S20a) are mostly constituted of GNR. They all show the typical fingerprint of the GNR absorption shape (spectrum in Figure S7). And second, the pulse (represented by a red Gaussian-shaped line with a $15 \mathrm{~nm}$ fwhm in Figure S20b) at $500 \mathrm{~nm}$ is exciting at a spectral position where the absorptivity of probes is at their lowest (i.e. $\mathcal{1} \sim 1000-3000$ L.mol. ${ }^{-1} \mathrm{~cm}^{-1}$ ) and absorptivity of the GNR is at its highest (i.e. $\varepsilon=23000$ L.mol. ${ }^{-1} \mathrm{~cm}^{-1}$ ). Concurrently, the selective excitation of the probes is easily achieved by selecting a wavelength where the graphitic material does not absorb.

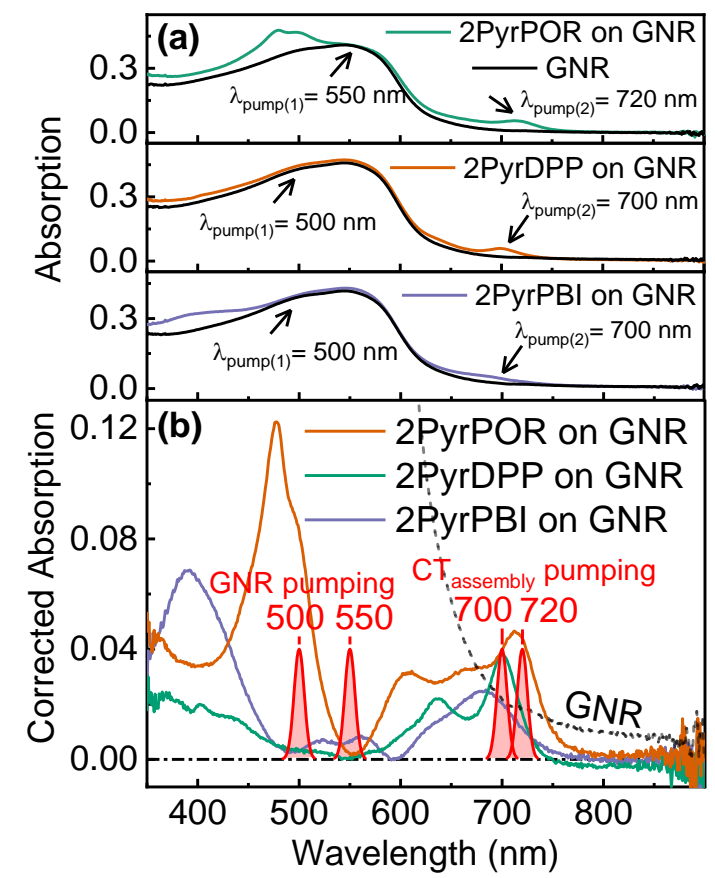

Figure S20. (a) Comparison of the absorption spectra between the dye-GNR assemblies (2PyrPOR (green), 2PyrDPP (red) and 2PyrPBI (blue)) and pure GNR (black) used for fsTAS. (b) Comparison of the resulting curves obtained by subtracting the absorption spectra of the free GNR from that of GNR-dye assemblies, with that of free GNR (black ----). The peaks at $700 \mathrm{~nm}$ (2PyrDPP and 2PyrPBI) and $720 \mathrm{~nm}$ (2PyrPOR) are the positions where selective excitation of the CT assembly is maximum. The peaks at 500 (2PyrDPP and 2PyrPBI) and 550 (2PyrPOR) $\mathrm{nm}$ are the positions where the free GNR absorption is large compared to that for the CT assemblies. Note that the fwhm of the laser pulse is $15 \mathrm{~nm}$ (indicated by red Gaussian-shaped peaks). 


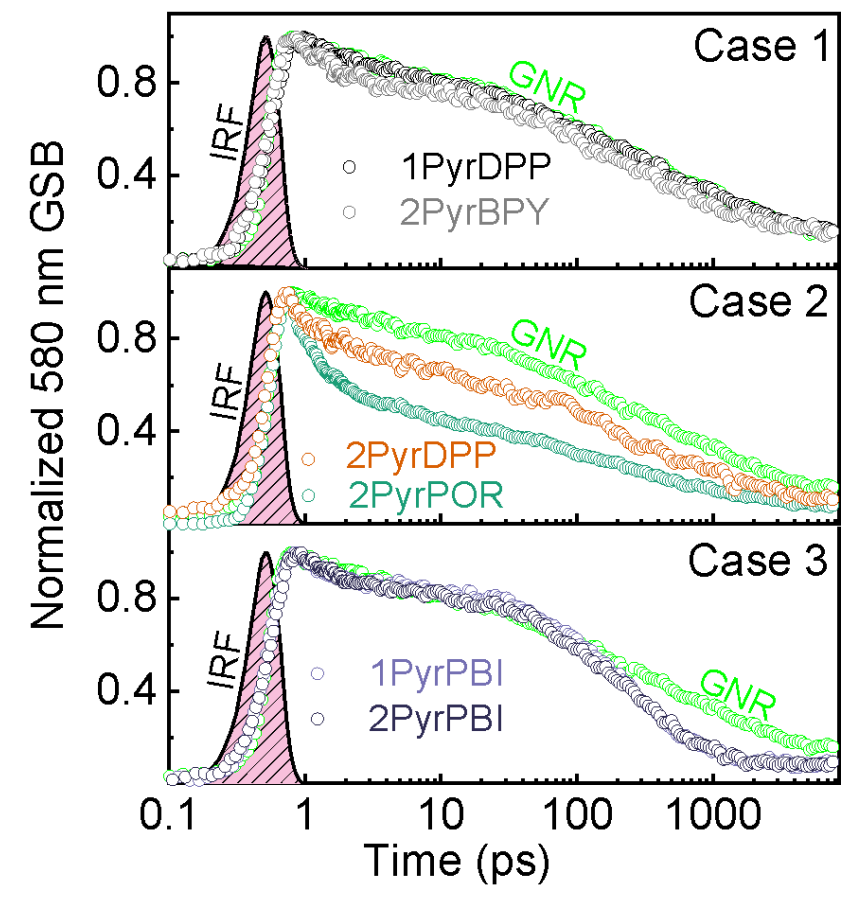

Figure S21. Time-evolution of the GSB band of GNR* (semi log scale) for the three cases and six CT assemblies adsorbed on GNR in $\mathrm{CHCl}_{3} ; \lambda_{\text {exc }}=500 \mathrm{~nm}$, except $\lambda_{\text {exc }}=550 \mathrm{~nm}$ for 2 PyrPOR. 

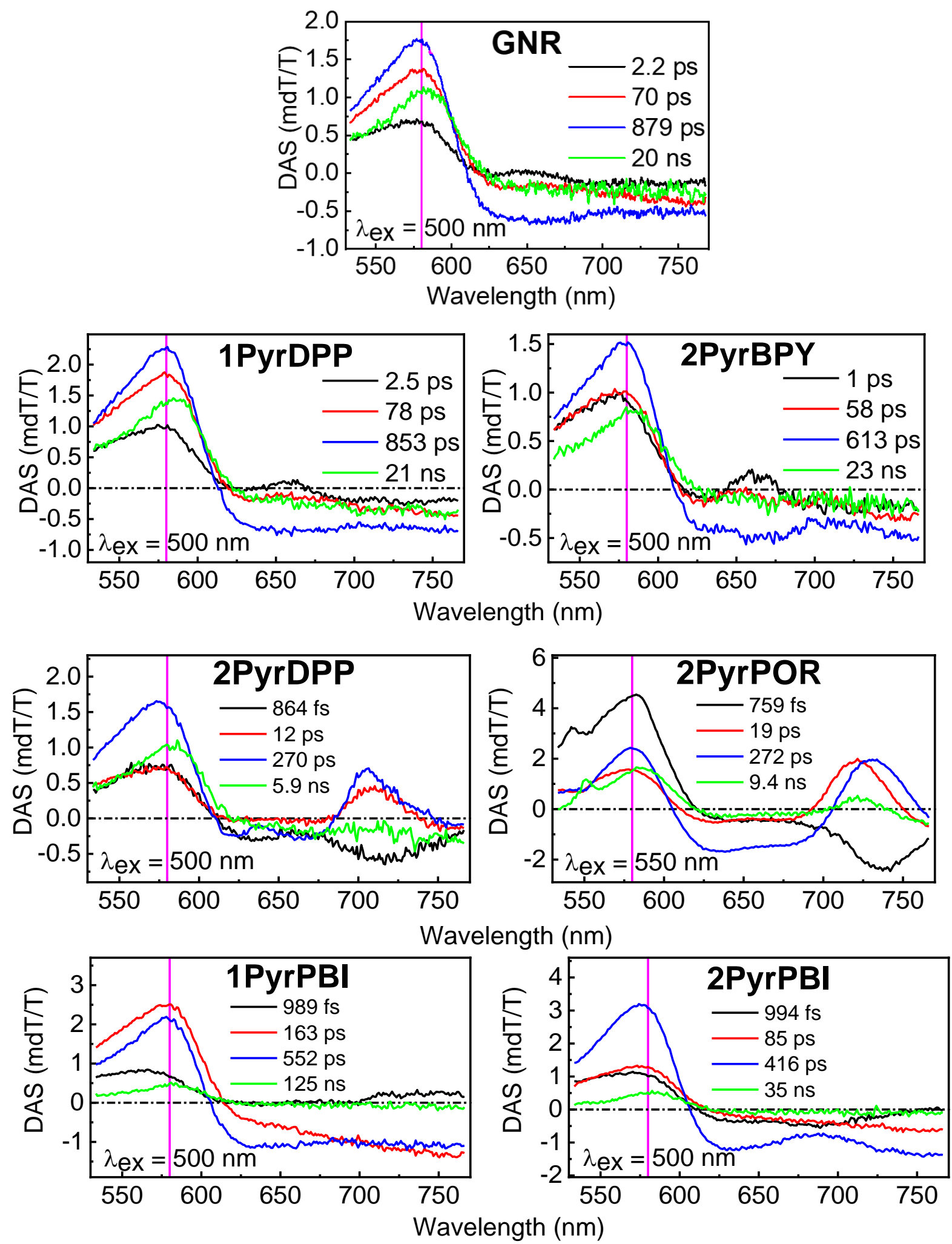

Figure S22. DAS of probes adsorbed on GNR and pristine GNR $\left(\lambda_{\text {exc }}=500 \mathrm{~nm}\right.$, except for $2 \mathrm{PyrPOR}$, which $\lambda_{\text {exc }}=550 \mathrm{~nm}$ ) in $\mathrm{CHCl}_{3}$. The purple line is the reported GSB of Figure S21. 
Explanation of the notion of "GSB transfer". Indeed, it is the only way that global analysis can extrapolate a rise from the mathematical deconvolution of the TA spectrum. In order to be able to fit the delay between the beginning of the measurement (at $\mathrm{t}=\mathrm{t}_{0}$ ) and the subsequent rise of the CT assembly GSB (Figure S23a), the first calculated DAS turns out to be an inverted CT GSB ( 738 nm, black DAS, Figure S23b). Physically, this signal translates into the buildup of the intensity of the CT GSB. Once fully generated, the GSB of this assembly can decay following a combined 19 and 272 ps lifetimes (red and blue DAS, Figure S23b). Due solely to this "negative CT GSB", the linear combination of fit) the reported kinetics (Figure S23c).

\section{(a) Raw 3D TA spectrum}

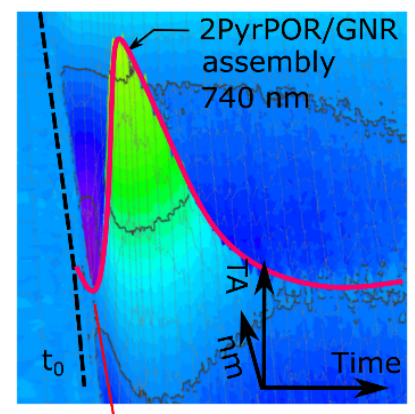

global analysis deconv (b) Decays Associated spectra

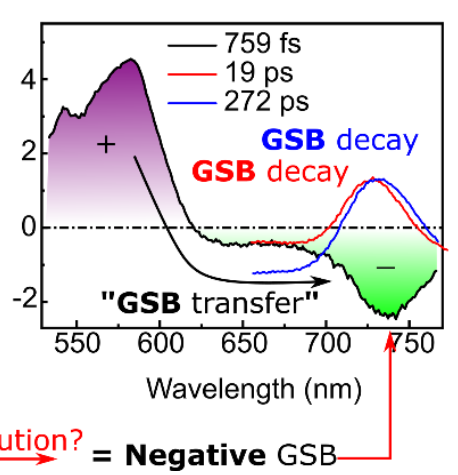

(c) Observed GSB dynamics

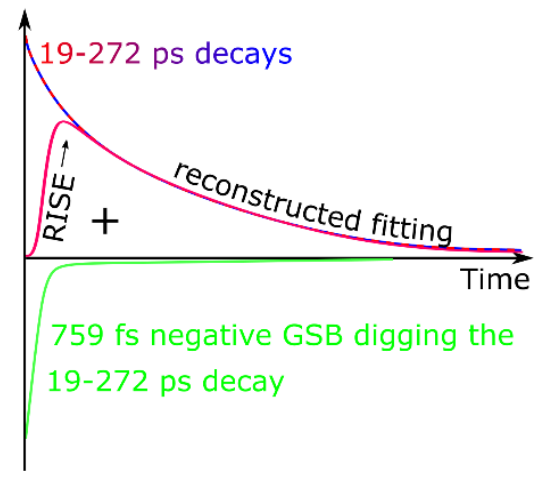

Figure S23. (a) Time-evolution of the GSB signal of the 2PyrPOR/GNR CT assembly in $\mathrm{CHCl}_{3}$ (b) DAS featuring the GSB population transfer and the two decays from the 19 and 272 ps windows (c) Rebuilt from the rise and decay traces of the GSB. 


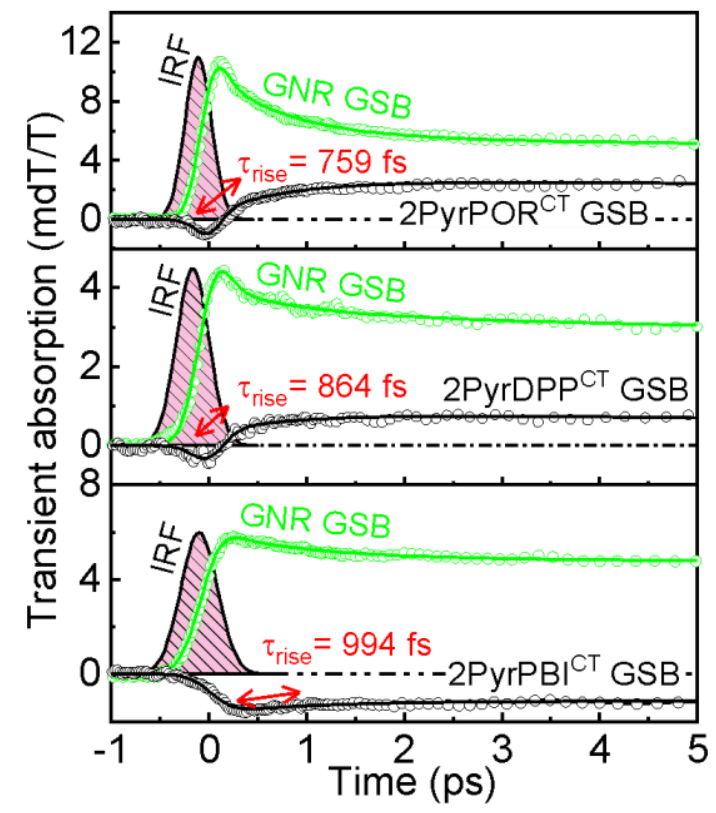

Figure S24. Time evolution of the GNR GSB (green) and CT complex GSB (black) intensities of $2 \mathrm{PyrPOR} / \mathrm{GNR}\left(\lambda_{\mathrm{exc}}=550 \mathrm{~nm}\right), 2 \mathrm{PyrDPP} / \mathrm{GNR}\left(\lambda_{\mathrm{exc}}=500 \mathrm{~nm}\right)$ and $2 \mathrm{PyrPBI} / \mathrm{GNR}\left(\lambda_{\mathrm{exc}}=500 \mathrm{~nm}\right)$. The selected time profiles are extracted from the $6 \mathrm{GSB}$ maxima reported in Figure S21. Circles are raw data and lines are the fit from global analysis. 


\section{Calculations of the relative amount of CT GSB arising from the visible ET process}

The transient absorption spectra $\mathrm{TA}(\lambda, \mathrm{t})$, which are $3 \mathrm{D}$ spectra are simplified to $2 \mathrm{D}$ spectra thanks to global analysis by mathematical deconvolution according to eq. 2 :

$$
T A(\lambda, t)=D A S_{1}(\lambda) * \tau_{1}(t)+D A S_{2}(\lambda) * \tau_{2}(t)+D A S_{3}(\lambda) * \tau_{3}(t)+D A S_{4}(\lambda) * \tau_{4}(t)
$$

where $\tau_{\mathrm{i}}$ is the lifetime of its respective decay associated spectra i DAS $\mathrm{S}_{\mathrm{i}}$. The three TAS reported in Figure 7a-c (2PyrPOR, 2PyrDPP and 2PyrPBI) all exhibit a transient $(\approx 650$ $750 \mathrm{~nm}$; black line) featuring the mirror image of the CT state GSB. It is an unambiguous mathematical characteristic of a DAS featuring an energy transfer. This energy transfer is, however, not the only source of energy promoting the population of the CT state. Indeed, the intensity of the CT state GSB signal in the DAS ${ }_{1}$ is too weak to be the sole source. There is first a population growth occurring within the laser pulse (within $135 \mathrm{fs}$ ), which is too fast to be accurately acquired by our system. Then a rise of the CT state GSB due to the energy transfer GNR $\rightarrow$ CT state GSB. The intensity of this rise is described by the $\mathrm{DAS}_{1}$ (black arrows; Figure S25c-e). Then, the CT state decays following the three components DAS $2, \mathrm{DAS}_{3}$ and DAS 4 (Figure S25a) having their individual intensities in colored arrows (Figure S25e-h).

The calculation of the initial population contribution of the CT state by $\mathrm{DAS}_{1}$ was simply performed by comparing the relative intensities of DAS 1 to $\mathrm{DAS}_{2}, \mathrm{DAS}_{3}$ and DAS $\mathrm{D}_{4}$. These calculations were easily performed taking advantage of the featureless transients' signal of the GNR (Figure S25b). The initial relative intensity (in percent) of the CT state GSB built up by the rise was calculated according to eq. 3 :

$$
\%=\frac{I_{D A S 1}}{I_{D A S 1}+I_{D A S 2}+I_{D A S 3}}(3)
$$

where IDASi stands for the intensity of the GSB of the CT state species of the $i^{\text {th }}$ DAS number i (Figure S25c-h). Note that a baseline (colored dashed line) was used in each calculation on Figure S25d-g to consider only the influence of the CT State GSB in the intensity calculation. 
(a)

Energy transfer within the 135 fs pulse
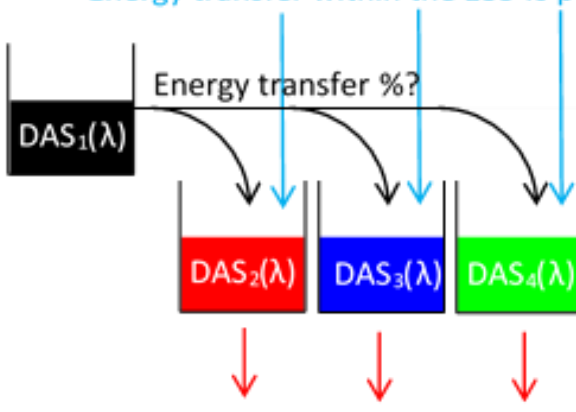

Decays of all GSBS
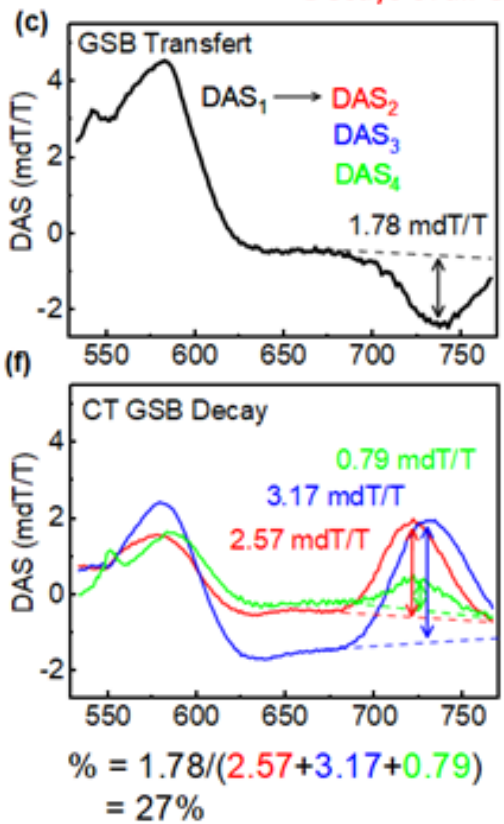

$=27 \%$ (b)
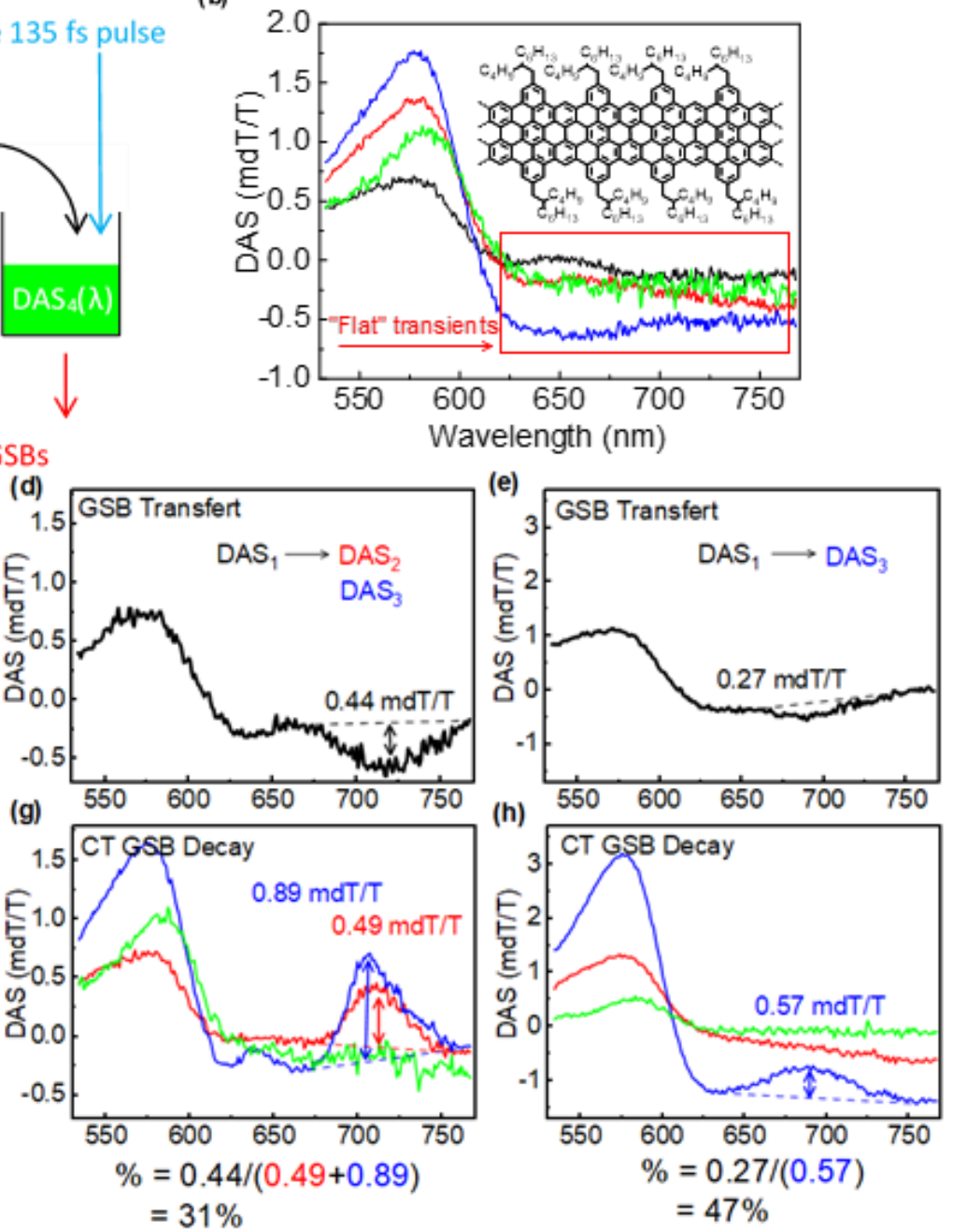

Figure S25. (a) Cartoon describing the communication channels explaining the origin of the intensity of the initial CT state GSB signal of the CT state. (b) DAS of GNR. The rectangle stresses the lack of signal of the GNR GSB in this region. This feature renders the calculations of the \% of eq. 3 more reliable. (c-e) Relative intensity (black arrow) of the GSB signal of the CT state from DAS ${ }_{1}$ (black line), which is treated as the population input arising from the energy transfer (f-h). Relative intensities (colored arrows) of the GSB signal of the CT state from $\mathrm{DAS}_{2}$ (red line), $\mathrm{DAS}_{3}$ (blue line) and $\mathrm{DAS}_{4}$ (green line). The sum of the colored arrows is treated as the initial population of the CT state. 


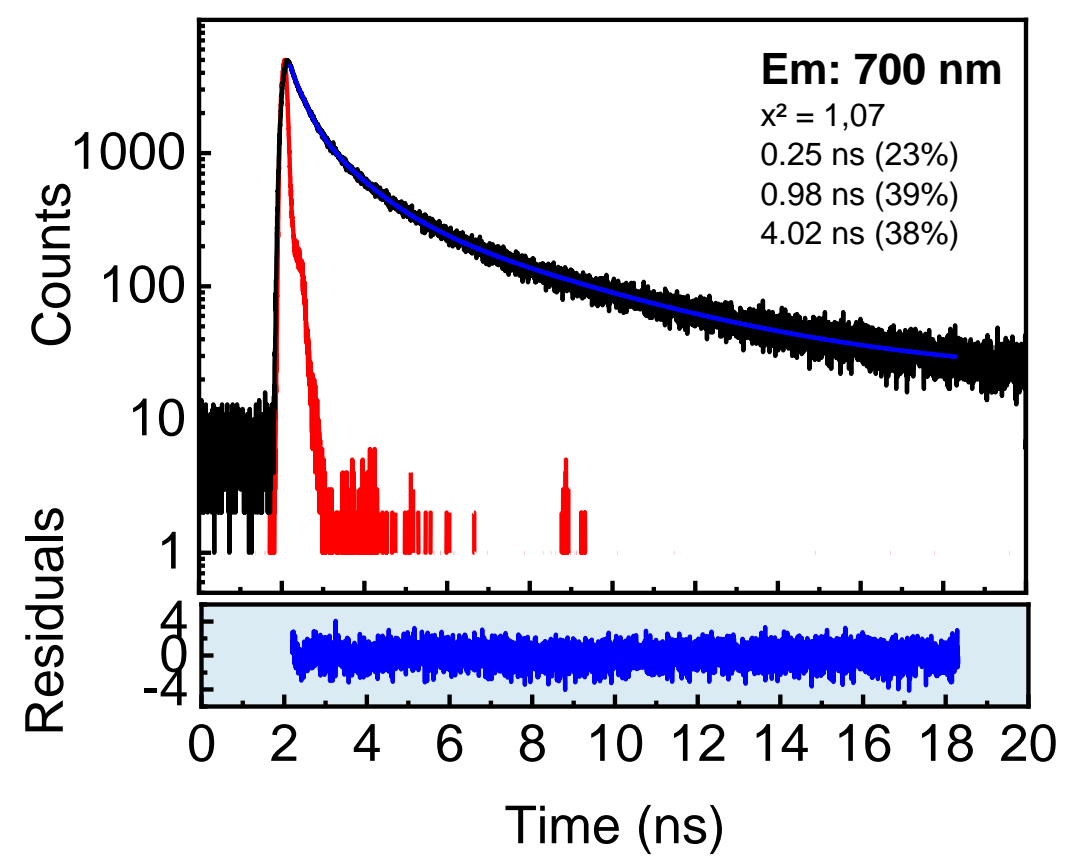

Figure S26. Fluorescence decay traces (black traces) and their lifetimes of $\mathrm{GNR}$ in $\mathrm{CHCl}_{3}$ at $273 \mathrm{~K}\left(\lambda_{\mathrm{exc}}=477 \mathrm{~nm}\right)$. Red trace $=$ pulse profile. Blue trace $=$ residual. The solutions were degassed upon bubbling $\operatorname{Ar}(\mathrm{g})$ for $5 \mathrm{~min}$.

\begin{tabular}{c|cccccccc} 
& $\begin{array}{c}\text { probe } \\
(\mathrm{mdT} / \mathrm{T})\end{array}$ & $\begin{array}{c}\mathrm{GNR} \\
(\mathrm{mdT} / \mathrm{T})\end{array}$ & probe/GNR & $\varepsilon_{\text {probes }}$ & $\varepsilon_{\mathrm{CT}^{\mathrm{a}}}$ & $\varepsilon_{\mathrm{GNR}}$ & $\varepsilon_{\mathrm{CT} / \mathrm{GNR}}$ & $\begin{array}{c}\text { probe: } \\
\mathrm{GNR}_{\text {monomer }}\end{array}$ \\
\hline 2PyrPOR & 15 & 8 & 0.533 & $63000(682 \mathrm{~nm})$ & $45000(715 \mathrm{~nm})$ & $23000(550 \mathrm{~nm})$ & 1.957 & 1.043 \\
2PyDPP & 7 & 4 & 0.571 & $45000(630 \mathrm{~nm})$ & $45000(700 \mathrm{~nm})$ & $23000(550 \mathrm{~nm})$ & 1.957 & 1.118 \\
2PyrPBI & 1.5 & 7 & 4.667 & $24000(627 \mathrm{~nm})$ & $26000(686 \mathrm{~nm})$ & $23000(550 \mathrm{~nm})$ & 1.130 & 5.275
\end{tabular}

Table S1. Calculation of the probe:GNR monomer ratio. ${ }^{\text {a }}$ The variation of the absorptivity upon CT state formation is calculated based on the proportional variation of the absorption spectra shown in Figure 3. 

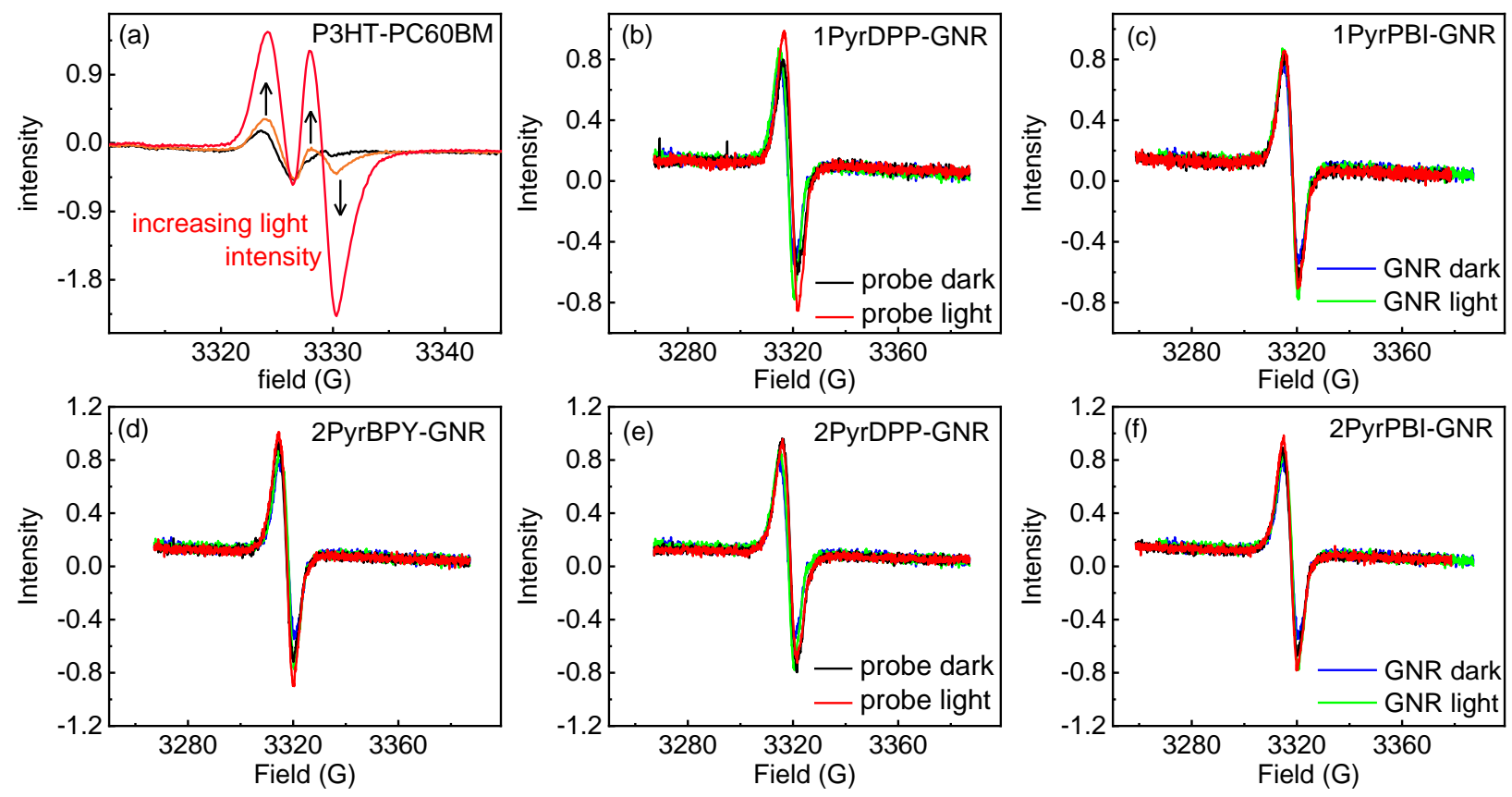

Figure S27. (a) ESR spectra of P3HT-PC $60 \mathrm{BM}$ (solid state, 149K) used as a control experiment demonstrating the possibility of monitoring the CS state formation under illumination $\left(\lambda_{\text {exc }}=532 \mathrm{~nm}\right.$; Power $\left.\approx 300 \mathrm{~mW}\right)$ with this custom setup. (b-f) ESR spectra of 2PyrBPY, 1PyrDPP, 2PyrDPP, 1PyrPBI and 2PyrPBI (toluene, 130K) in the dark (black line) and with illumination (red line). ESR spectra of GNR in the dark (blue line) and with illumination (green line).

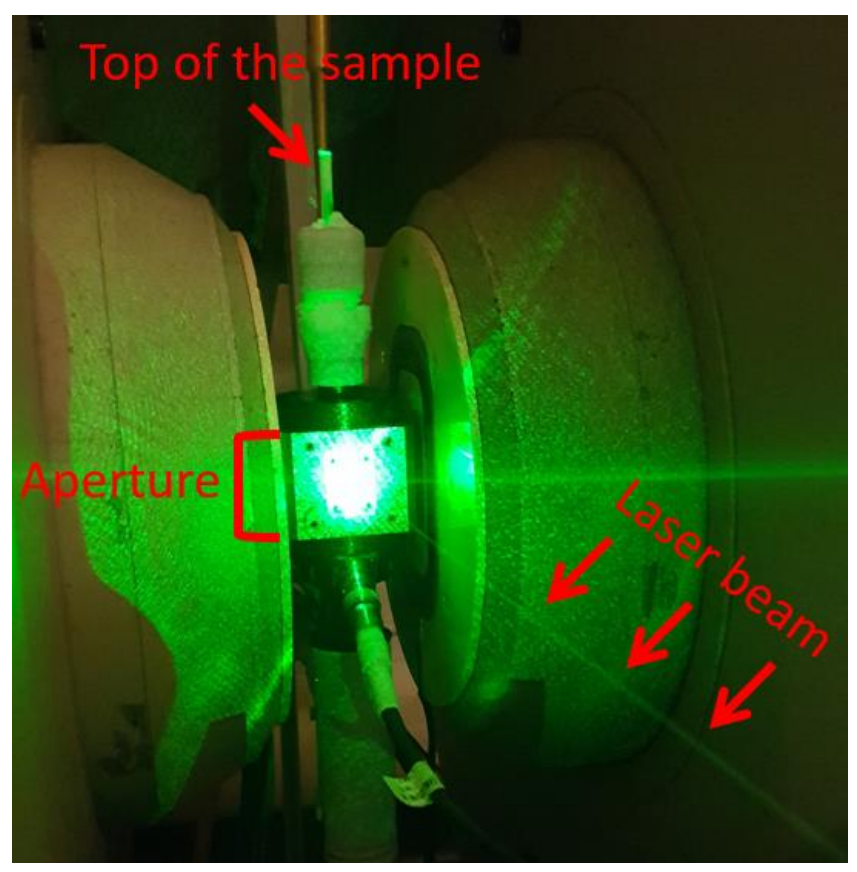

Figure S28. Picture of the ESR setup under light exposition. 


\section{Materials and instruments}

All reagents and solvents were purchased from Sigma-Aldrich, Oakwood Chemicals or Fisher chemicals. Gelatin was obtained from Knox ${ }^{\circledR}$. Reduced graphene oxide ${ }^{1}, 5,15-$ diethynyl-10,20-bis(5-(2-butyloctyl)thienyl)porphyrin zinc $^{2}, \quad$ iodopyrene ${ }^{3}, \quad$ ethynyl pyrene $^{4}$, 3,6-bis(5-bromo-2-thienyl)-2,5-bis(2-butyloctyl)-2,5-dihydropyrrolo[3,4c]pyrrole-1,4-dione,$\quad 3$-(5-bromo-2-thienyl)-2,5-bis(2-butyloctyl)-2,5-dihydro-6-(2thienyl)pyrrolo[3,4-c]pyrrole-1,4-dione ${ }^{6}, \quad$ N,N'-bis(2-ethylhexyl)-1-bromo-3,4,9,10perylene diimide ${ }^{7}, \mathrm{~N}, \mathrm{~N}^{\prime}$-bis(2-ethylhexyl)-1,7-dibromo-3,4,9,10-perylene diimide ${ }^{7}$, boron, difluoro[3-iodo-5-[1-(4-iodo-3,5-dimethyl-2H-pyrrol-2-ylidene-kN)hexadecyl]-2,4dimethyl-1H-pyrrolato- $\mathrm{kN}]$ (extracted from the monobromation batch), boron, [2-[1-(3,5dimethyl-2H-pyrrol-2-ylidene-kN)hexadecyl]-3,5-dimethyl-1H-pyrrolato-kN]difluoro ${ }^{8}$, 2-butyloctylbromide ${ }^{9}$, 3-bromobenzil ${ }^{10,11}$ were synthesized according to procedures previously reported in the literature. Toluene, diisopropylamine, triethylamine used for Sonogashira reactions were distilled $2 \mathrm{~h}$ before use and dried respectively on sodium wire, $\mathrm{CaH}_{2}$ and $\mathrm{CaH}_{2}$. The $\mathrm{CH}_{2} \mathrm{Cl}_{2}$ used for the GNR synthesis was distilled $2 \mathrm{~h}$ on $\mathrm{CaH}_{2}$ before use.

The ${ }^{1} \mathrm{H},{ }^{13} \mathrm{C}$ and COSY NMR spectra were performed on either a Bruker Avance NEO 400 MHz NMR (actively shielded Oxford Instruments magnet) or a Bruker Avance III HD 300 MHz ultrashield NMR. ESR spectra were recorded on a Bruker Elexsys E580 X-band EPR Spectrometer. The high-resolution accurate mass spectra were acquired on a maXis $3 \mathrm{G}$ (ESI-QqTOF) orthogonal mass spectrometer from Bruker Daltonik (Bremen, Germany) using electrospray ionization or atmospheric pressure chemical ionization in positive ion mode. The mass spectra were recorded in the range $\mathrm{m} / \mathrm{z} 50-1200$ and the external calibration was performed using a sodium formate $0.5 \mathrm{mM}$ solution. The absorption spectra were recorded on a Varian Carry 300 Bio. The fluorescence lifetimes were measured on a FLS9800 phosphorimeter using a $477 \mathrm{~nm}$ picosecond pulsed diode laser as an external excitation source (model DD-485L). The photon counts were recorded with a timecorrelated single-photon counting (TCSPC) system on the FLS980. All emission measurements were performed on a QuantaMaster 400 phosphorimeter from Photon Technology International. A Xenon lamp was used for excitation and a NIR PMT-7-B detector was used to record emission. The instrument response was corrected in fluorescence spectra. The $\mathrm{M}_{\mathrm{n}}, \mathrm{M}_{\mathrm{w}}$ and the polydispersity index of the GNR precursor were obtained using standard polystyrene calibration. They were determined using a gel permeation chromatography (GPC) system from an Agilent Technologies 1260 Infinity 2017 using a $50 \mu \mathrm{m}$ MiniMIX-C column set. The GPC experiment was run in THF at 30 ${ }^{\circ} \mathrm{C}$ with a $0.4 \mathrm{~mL} \cdot \mathrm{min}^{-1}$ flow. A $100 \mu \mathrm{L}$ injection of a $1 \mathrm{mg} \cdot \mathrm{mL}^{-1} \mathrm{GNR}$ precursor solution was measured using viscosity and refractive index. Cyclic voltammetry experiments were performed with a PAR 273 potentiostat. Transmission and scanning electron microscopy images were respectively recorded on a H-7500 and a S-4700 from Hitachi. 


\section{Transient absorption spectroscopy}

\section{Set-up}

The fs-transient spectra and decay profiles were acquired on a custom-built set-up using an amplified Ti:sapphire laser source (Model Solstice from Spectra Physics, $795 \mathrm{~nm}, 1 \mathrm{kHz}$, $3.5 \mathrm{~W}$, fwmh $=75 \mathrm{fs}$ ). The pump excitation wavelengths were tuned using an OPA (model 800 CF from Spectra Physics). The white light continuum was generated inside a sapphire window and was spitted into a probe and a reference beam. Both signals were measured by a custom-made dual line CCD camera (S7030, Spectronic Devices). The delay line permitted one to probe up to $8 \mathrm{~ns}$ with an accuracy of $\sim 4 \mathrm{fs}$. Both pump ( $250 \mu \mathrm{m})$ and probe $(\sim 70-110 \mu \mathrm{m})$ beams were focused non-colinearly on the sample. All recorded full transient 3D absorption spectra are deconvoluted by global analysis using Glotaran ${ }^{12}$. The instrument response function (IRF) and the temporal dispersion of the probe are deconvoluted to converge to the actual 3D image of the original experimental data. The regression made during global analysis follows equation 1.

$$
T A(\lambda, t)=D A S_{1}(\lambda) e^{\frac{t}{\tau 1}}+\cdots+D A S_{n}(\lambda) e^{\frac{t}{\tau n}}
$$

Where decays associated spectra $\left(\operatorname{DAS}_{\mathrm{i}}(\lambda)\right)$ which are the spectra lineshape associated with a specific lifetimes $\mathrm{i}\left(\tau_{\mathrm{i}}\right) .2$ and $3 \mathrm{DAS}_{\mathrm{i}}(\lambda)$ were found best to report RGO CT states TA spectra while 4 DAS $_{\mathrm{i}}(\lambda)$ were more appropriate to report the GNR CT States TA spectra.

\section{Preparation of solid-state gelatin films of probes adsorbed on RGO}

The supramolecular complexes of RGO and probes were stabilized in the solid-state by forming film made of gelatin. Typically, to a solution containing the probe $(\mathrm{A}=0.1$ at the maximum intensity of the CT band, path length $=1 \mathrm{~cm})$ in THF $(2100 \mu \mathrm{L})$ was added an RGO dispersion in water ( $\approx$ typically $2000 \mu \mathrm{L}$ ) until the full formation of the CT species. This was achieved by monitoring the CT state band formation by absorption spectroscopy. The water content was adjusted to a total water amount of $7000 \mu \mathrm{L}$ and a maximum of THF was removed under reduced pressure. Note: Failure to remove the THF would lead to blurred films. $1 \mathrm{~g}$ on gelatin was added and the mixture was allowed to stir at $45^{\circ} \mathrm{C}$ for 30 min until the solution became translucent. The solution was drop casted with a Pasteur pipette on a piece of glass and was permitted to set for $3 \mathrm{~h}$. Deposition on rough substrates would also lead to blurred films. The transparent films were peeled off and exhibited a thickness of $\approx 500 \mu \mathrm{m}$ suitable for TA analysis.

\section{Preparation of solution samples of probes adsorbed on GNR}

Solutions of concentrated probes adsorbed on $\mathrm{GNR}$ in $\mathrm{CHCl}_{3}$ were stable for several days. Typically, to a solution of free probe $\left(A=0.1\right.$ at the maximum intensity of the $S_{0} \rightarrow S_{1}$ band, path length $=1 \mathrm{~mm})$ in $\mathrm{CHCl}_{3}(300 \mu \mathrm{L})$ was progressively added a solution of GNR in $\mathrm{CHCl}_{3}(\approx$ typically 8-10 $\mu \mathrm{L})$. The emission of the solution was monitored and GNR 
addition was stopped once no more emission of free probes was observed upon excitation at $500 \mathrm{~nm}$. A custom-made magnetic stirrer ( $<1 \mathrm{~mm}$ thick) was added. The cell was sealed with a Teflon cork and ready for TA analysis. For each probe on GNR samples, a corresponding pristine GNR sample with an identical quantity of GNR was prepared as control experiments.

\section{Computational method}

\section{Ground state}

All electronic calculation and structure optimizations have been carried out by DFT with the Quantum Espresso v6.3 code $^{13,14}$. The PBE functional ${ }^{15}$ have been used in conjunction with the pbe-n-kjpaw_psl.UPF PAW pseudopotentials ${ }^{16}$ retrieved from the Quantum Espresso pseudo-potentials website (https://www.quantum-espresso.org/) Beforehand, GNR (308 atoms) and 2PyrDPP (86 atoms) have been optimized separately. The complexed structure (ADS, 394 atoms) has been obtained by performing an optimization of the previous calculation together. Studies have been produced to ensure that the energy converged in respect to the size of the cell, the energy cutoff for the wavefunction (ecutwfc) and the number of k-points (K_POINTS). The energy cutoff for the wavefunction and the kinetic energy cutoff for the charge density have been set to $40 \mathrm{Ry}$ and $320 \mathrm{Ry}$ respectively. The final dimensions of the 2 PyrDPP and GNR system is $55.711 \AA$ x $40 \AA$ x $20 \AA$.

\section{Excited state}

The TD-DFT calculations were performed with the turbo_lanczos.x module included in the Quantum Espresso software suite ${ }^{17,18}$. The PBE functional have also been used, but because of an implementation restriction with the pbe-n-rrkjus_psl.1.0.0.UPF Ultra soft pseudo-potentials ${ }^{16} 500$ Lanczos iterations have been performed to keep a reasonable amount of computational time even with the biggest system. Finally, the charge response has been computed at the less energetic peak in the computed absorption spectrum obtained with the turbo_spectrum.x executable. 


\section{Synthesis}

\section{PyrPOR}
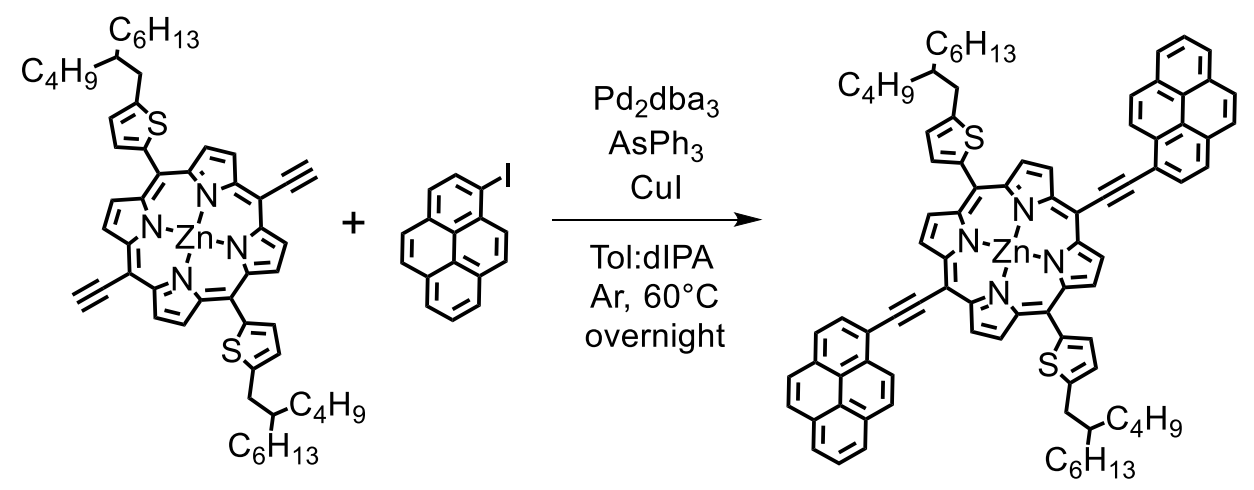

2PyrPOR was synthesized using a Sonogashira under Glaser-Hay free coupling conditions already reported by our group ${ }^{19}$. 5,15-Diethynyl-10,20-bis(5-(2-butyloctyl)thienyl)porphyrin zinc $(75 \mathrm{mg}, 0.070 \mathrm{mmol})$ and iodopyrene $(69.2 \mathrm{mg}, 0.211 \mathrm{mmol})$ were dissolved in a degassed and argon protected solution of toluene $(170 \mathrm{~mL})$ and diisopropylamine $(5.3 \mathrm{~mL})$. The temperature was adjusted to $60^{\circ} \mathrm{C}$ and $\mathrm{Pd}_{2} \mathrm{dba}_{3}(12.9 \mathrm{mg}$, $0.014 \mathrm{mmol}$ ) and $\mathrm{AsPh}_{3}(64.6 \mathrm{mg}, 0.21 \mathrm{mmol})$ were directly added as solid. Briefly, a 1 $\mathrm{mL}$ syringe was filled with catalysts, purged three times with argon, poured in the solution, and fill in and out repeatedly until all catalysts were transferred to the solution. The solution was stirred overnight. The mixture was poured in $300 \mathrm{~mL}$ of $\mathrm{MeOH}$ and set aside for $2 \mathrm{~h}$. The green precipitate was filtered, washed with methanol to give $29 \%$ of $2 \mathrm{PyrPOR}(27 \mathrm{mg}$, $0.020 \mathrm{mmol}$ ) as a weakly soluble green solid. Several drops of pyridine were needed to fully dissolve $2 \mathrm{PyrPOR}$ in a reasonable amount a solvent. ${ }^{1} \mathrm{H} \mathrm{NMR}\left(\mathrm{CDCl}_{3}, 300 \mathrm{MHz}\right): \delta_{\mathrm{H}}$ $9.89(\mathrm{~d}, 4 \mathrm{H}, J=4.5 \mathrm{~Hz}), 9.22(\mathrm{~d}, 4 \mathrm{H}, J=4.5 \mathrm{~Hz}), 8.95(\mathrm{~d}, 2 \mathrm{H}, J=8.9 \mathrm{~Hz}), 8.46(\mathrm{~d}, 2 \mathrm{H}, J$ $=7.8 \mathrm{~Hz}), 8.15-7.69(\mathrm{~m}, 16 \mathrm{H}), 7.24(\mathrm{~d}, 2 \mathrm{H}), 3.18(\mathrm{~d}, 4 \mathrm{H}, J=6.7 \mathrm{~Hz}) ;{ }^{13} \mathrm{C} \mathrm{NMR}\left(\mathrm{CDCl}_{3}\right.$, $76 \mathrm{MHz}) \delta_{\mathrm{C}} 152.3,150.9,149.2,147.0,141.5,136.1,133.3,132.6,131.7,130.9,129.5$, 128.1, 126.8, 126.0, 125.4, 124.5, 123.7, 118.7, 114.8, 102.2, 99.7, 96.1, 40.6, 35.2, 33.9, $33.6,32.2,30.0,29.3,27.0,23.4,23.0,14.5,14.4,1.2$;

\section{PyrDPP}

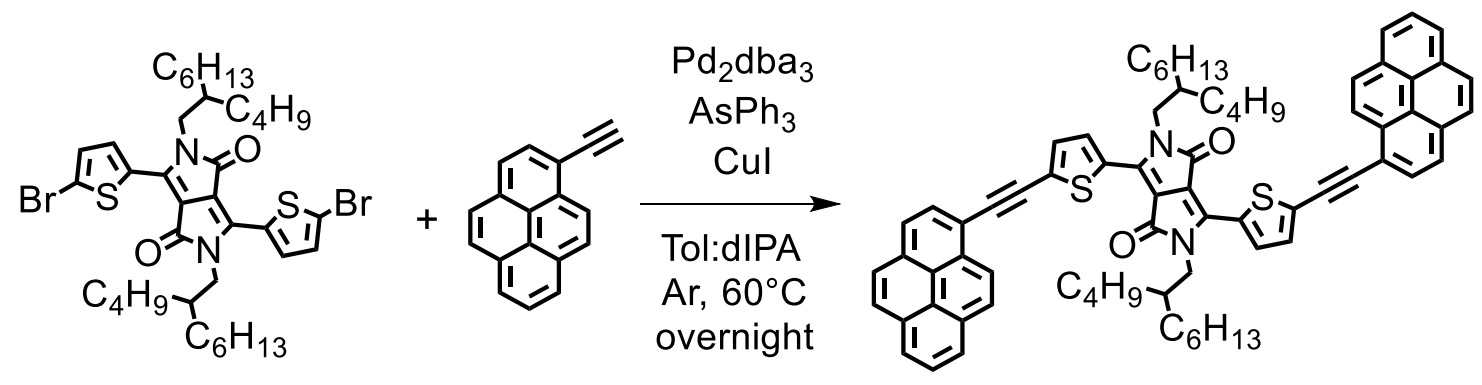


2PyrDPP was synthesized using a Sonogashira under Glaser-Hay free coupling conditions already reported by our group ${ }^{19}$. 3,6-Bis(5-bromo-2-thienyl)-2,5-bis(2-butyloctyl)-2,5dihydropyrrolo[3,4-c]pyrrole-1,4-dione $(100 \mathrm{mg}, 0.126 \mathrm{mmol})$ and ethynyl pyrene (56.9 $\mathrm{mg}, 0.251 \mathrm{mmol}$ ) were dissolved in a degassed and argon protected solution of toluene (76 $\mathrm{mL})$ and diisopropylamine $(2.4 \mathrm{~mL})$. The temperature was adjusted to $60^{\circ} \mathrm{C}$ and $\mathrm{Pd}_{2} \mathrm{dba}_{3}$ $(23.1 \mathrm{mg}, 0.0252 \mathrm{mmol})$ and $\mathrm{AsPh}_{3}(116 \mathrm{mg}, 0.378 \mathrm{mmol})$ were directly added as solid. A $1 \mathrm{~mL}$ syringe was filled with catalysts, purged three times with argon, poured in the solution, and fill in and out repeatedly until all catalysts were transferred to the solution. The solution was stirred overnight. The mixture was poured in $400 \mathrm{~mL}$ of $\mathrm{MeOH}$ and set aside for $2 \mathrm{~h}$. The blue precipitate was filtered and washed with methanol. The product was purified by recrystallization in $\mathrm{CH}_{2} \mathrm{Cl}_{2}(1 \mathrm{~mL})$ and EtOAc $(50 \mathrm{~mL})$ at $-78^{\circ} \mathrm{C}$ to give $64 \%$ of 2PyrDPP ( $88 \mathrm{mg}, 0.0249 \mathrm{mmol})$ as a blue solid. ${ }^{1} \mathrm{H} \mathrm{NMR}\left(\mathrm{CDCl}_{3}, 300 \mathrm{MHz}\right): \delta_{\mathrm{H}} 9.95$ $(\mathrm{d}, 2 \mathrm{H}, J=4.1 \mathrm{~Hz}), 8.53(\mathrm{~d}, 2 \mathrm{H}, J=9.1 \mathrm{~Hz}), 8.24-7.94(\mathrm{~m}, 16 \mathrm{H}), 7.52(\mathrm{~d}, 2 \mathrm{H}, J=4.1 \mathrm{~Hz})$, $4.03(\mathrm{~d}, 4 \mathrm{H}, J=7.7 \mathrm{~Hz}), 2.07-1.91(\mathrm{~m}, 2 \mathrm{H}), 1.50-1.13(\mathrm{~m}, 32 \mathrm{H}), 0.98-0.74(\mathrm{~m}, 12 \mathrm{H}) ;{ }^{13} \mathrm{C}$ $\mathrm{NMR}\left(\mathrm{CDCl}_{3}, 76 \mathrm{MHz}\right) \delta_{\mathrm{C}} 161.6,139.5,135.9,133.1,132.0,131.9,131.3,131.1,130.8$, 129.6, 129.0, 128.8, 127.3, 126.5, 126.0, 124.7, 124.3, 116.7, 109.0, 97.6, 88.2, 53.6, 46.6, 38.0, 32.0, 31.4, 31.1, 29.9, 28.7, 26.4, 23.3, 22.8, 14.3; HRMS (APCI+) m/z: [M] ${ }^{+}$calcd for $\mathrm{C}_{74} \mathrm{H}_{72} \mathrm{~N}_{2} \mathrm{O}_{2} \mathrm{~S}_{2}, 1084.5030$; found, 1084.5053 .

\section{PyrDPP}

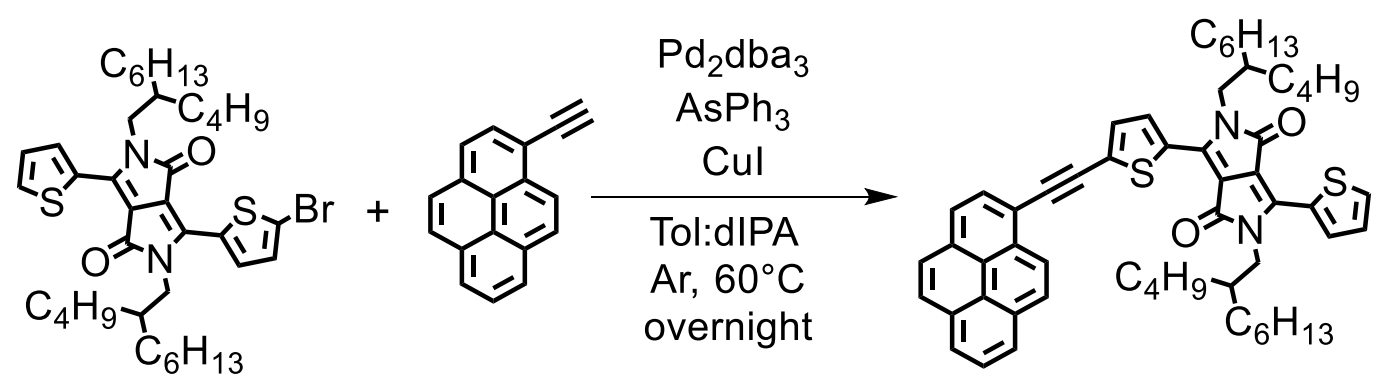

1PyrDPP was synthesized using a Sonogashira under Glaser-Hay free coupling conditions already reported by our group ${ }^{19}$. 3-(5-Bromo-2-thienyl)-2,5-bis(2-butyloctyl)-2,5-dihydro6-(2-thienyl)pyrrolo[3,4-c]pyrrole-1,4-dione (71 $\mathrm{mg}, 0.099 \mathrm{mmol})$ and ethynyl pyrene (45 $\mathrm{mg}, 0.19 \mathrm{mmol}$ ) were dissolved in a degassed and argon protected solution of toluene (240 $\mathrm{mL})$ and diisopropylamine $(7.4 \mathrm{~mL})$. The temperature was adjusted to $60^{\circ} \mathrm{C}$ and $\mathrm{Pd}_{2} \mathrm{dba}_{3}$ (18.2 $\mathrm{mg}, 0.019 \mathrm{mmol})$ and $\mathrm{AsPh}_{3}(91 \mathrm{mg}, 0.29 \mathrm{mmol})$ were directly added as solid. A 1 $\mathrm{mL}$ syringe was filled with catalysts, purged three times with argon, poured in the solution, and fill in and out repeatedly until all catalysts were transferred to the solution. The solution was stirred overnight. The mixture was washed with water $(3 \times 100 \mathrm{~mL})$, extracted with $\mathrm{CH}_{2} \mathrm{Cl}_{2}$ (3 x $100 \mathrm{~mL}$ ), dried on $\mathrm{MgSO}_{4}$ and the solvent was removed under reduced pressure. The crude product was purified by silica column chromatography (from 50 to $55 \%$ of $\mathrm{CH}_{2} \mathrm{Cl}_{2}$ in Hexanes) to yield $72 \%$ (62 mg, $0.072 \mathrm{mmol}$ ) of 1 PyrDPP as a blue solid (purple in solution). ${ }^{1} \mathrm{H} \mathrm{NMR}\left(\mathrm{CDCl}_{3}, 300 \mathrm{MHz}\right): \delta_{\mathrm{H}} 8.95-8.88(\mathrm{~m}, 2 \mathrm{H}), 8.59(\mathrm{~d}, 1 \mathrm{H}, J=$ 9.1 Hz), 8.29-7.99 (m, 8H), $7.64(\mathrm{~d}, 1 \mathrm{H}, J=4.8 \mathrm{~Hz}), 7.54(\mathrm{~d}, 1 \mathrm{H}, J=4.1 \mathrm{~Hz}), 7.31-7.26$ 
$(\mathrm{m}, 1 \mathrm{H}), 4.04(\mathrm{~d}, 4 \mathrm{H}, J=9.1 \mathrm{~Hz}), 2.12-1.81(\mathrm{~m}, 2 \mathrm{H}), 1.45-1.11(\mathrm{~m}, 32 \mathrm{H}), 0.94-0.76(\mathrm{~m}$, $12 \mathrm{H}) ;{ }^{13} \mathrm{C} \mathrm{NMR}\left(\mathrm{CDCl}_{3}, 76 \mathrm{MHz}\right) \delta_{\mathrm{C}} 161.8,140.8,139.5,135.6,135.5,133.0,132.1,132.0$, $131.4,131.2$, 130.9, 130.0, 129.7, 128.9, 128.8, 128.7, 128.6, 127.4, 126.6, 126.1, 125.4, 124.8, 124.6, 124.4, 116.8, 108.9, 108.3, 97.3, 88.1, 46.6, 38.0, 37.9, 32.0, 31.9, 31.4, 31.1, 29.9, 29.8, 28.7, 28.6, 26.4, 26.4, 23.2, 22.8, 14.2, 14.2; HRMS (ESI+) m/z: [M+Na] ${ }^{+}$calcd for $\mathrm{C}_{56} \mathrm{H}_{64} \mathrm{~N}_{2} \mathrm{NaO}_{2} \mathrm{~S}_{2}, 883.4301$; found, 883.4309.

\section{PyrPBI}

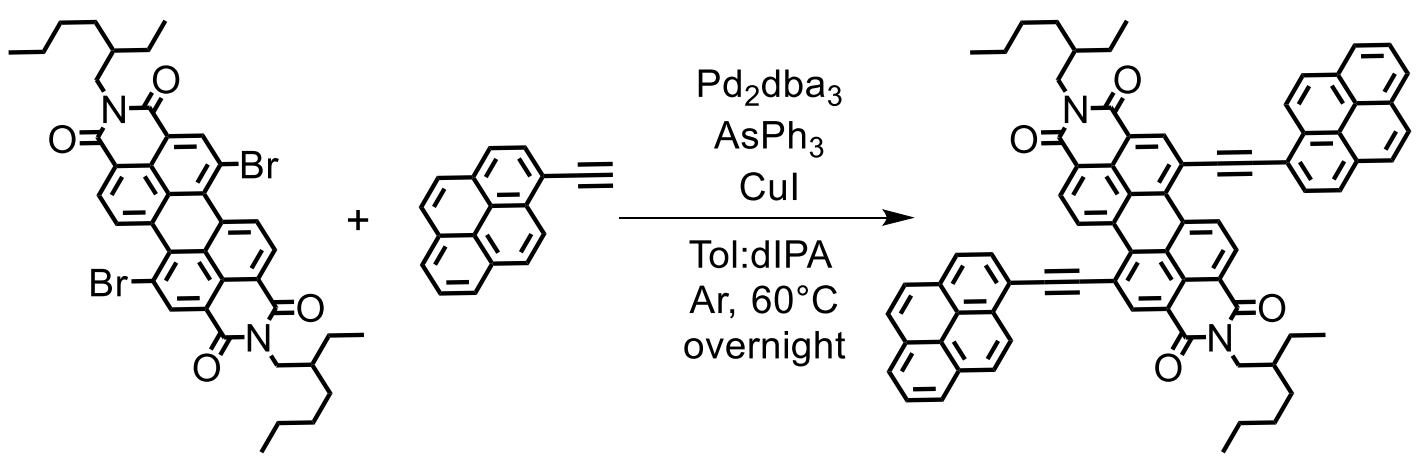

2PyrPBI was synthesized using a Sonogashira under Glaser-Hay free coupling conditions already reported by our group ${ }^{19}$. N,N'-Bis(2-ethylhexyl)-1,7-dibromo-3,4,9,10-perylene diimide (35 mg, $0.0452 \mathrm{mmol}$ ) and ethynyl pyrene $(31 \mathrm{mg}, 0.136 \mathrm{mmol}$ ) were dissolved in a degassed, and argon protected solution of toluene $(110 \mathrm{~mL})$ and diisopropylamine $(3.4$ $\mathrm{mL}$ ). The temperature was adjusted to $60^{\circ} \mathrm{C}$ and $\mathrm{Pd}_{2} \mathrm{dba}_{3}(8.3 \mathrm{mg}, 0.0090 \mathrm{mmol})$ and $\mathrm{AsPh}_{3}$ (42 mg, $0.136 \mathrm{mmol}$ ) were directly added as solid. A $1 \mathrm{~mL}$ syringe was filled with catalysts, purged three times with argon, poured in the solution, and fill in and out repeatedly until all catalysts were transferred to the solution. The solution was stirred overnight. The mixture was poured in $300 \mathrm{~mL}$ of $\mathrm{MeOH}$ and set aside for $2 \mathrm{~h}$. The blue precipitate was filtered, washed with methanol to give $55 \%$ of 2 PyrPBI $(27 \mathrm{mg}, 0.025 \mathrm{mmol})$ as a strongly aggregating blue solid soluble in hot chloroform. ${ }^{1} \mathrm{H} \mathrm{NMR}\left(\mathrm{CDCl}_{3}, 300 \mathrm{MHz}\right): \delta_{\mathrm{H}} 9.91(\mathrm{~d}$, $2 \mathrm{H}, J=8.1 \mathrm{~Hz}), 8.62(\mathrm{~s}, 2 \mathrm{H}), 8.45(\mathrm{~d}, 2 \mathrm{H}, J=8.1 \mathrm{~Hz}), 8.18(\mathrm{~d}, 2 \mathrm{H}, J=8.8 \mathrm{~Hz}), 7.91-7.4$ $(\mathrm{m}, 16 \mathrm{H}), 4.26(\mathrm{~m}, 4 \mathrm{H})$; HRMS (APCI+) $\mathrm{m} / \mathrm{z}:[\mathrm{M}]^{+}$calcd for $\mathrm{C}_{76} \mathrm{H}_{58} \mathrm{~N}_{2} \mathrm{O}_{4}, 1062.4391$; found, 1062.4412 .

\section{PyrPBI}

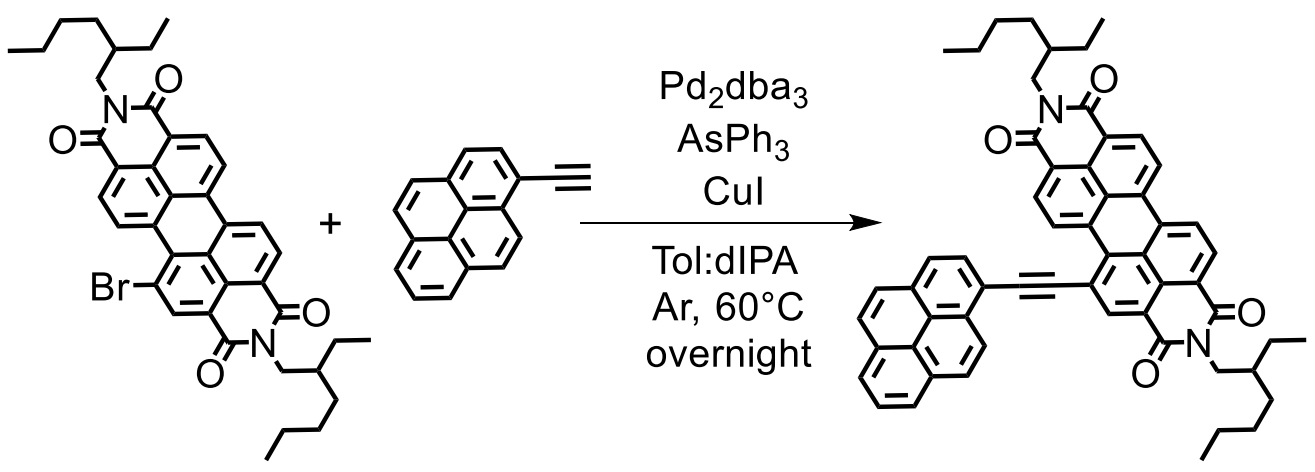


1PyrPBI was synthesized using a Sonogashira under Glaser-Hay free coupling conditions already reported by our group ${ }^{19}$. N,N'-Bis(2-ethylhexyl)-1-bromo-3,4,9,10-perylene diimide (50 mg, $0.0721 \mathrm{mmol}$ ) and ethynyl pyrene $(33 \mathrm{mg}, 0.144 \mathrm{mmol})$ were dissolved in a degassed, and argon protected solution of toluene $(175 \mathrm{~mL})$ and diisopropylamine (5.4 $\mathrm{mL}$ ). The temperature was adjusted to $60^{\circ} \mathrm{C}$ and $\mathrm{Pd}_{2} \mathrm{dba}_{3}(13 \mathrm{mg}, 0.0144 \mathrm{mmol})$ and $\mathrm{AsPh}_{3}$ (42 mg, $0.216 \mathrm{mmol}$ ) were directly added as solid. A $1 \mathrm{~mL}$ syringe was filled with catalysts, purged three times with argon, poured in the solution, and fill in and out repeatedly until all catalysts were transferred to the solution. The solution was stirred overnight. The mixture was poured in $500 \mathrm{~mL}$ of $\mathrm{MeOH}$ and set aside for $2 \mathrm{~h}$. The purple precipitate was filtered, washed with methanol to give $92 \%$ of 1 PyrPBI $(56 \mathrm{mg}, 0.067 \mathrm{mmol}$ ) as a purple solid. ${ }^{1} \mathrm{H}$ NMR $\left(\mathrm{CDCl}_{3}, 300 \mathrm{MHz}\right): \delta_{\mathrm{H}} 9.05(\mathrm{~d}, 1 \mathrm{H}, J=7.9 \mathrm{~Hz}), 7.83(\mathrm{~d}, 1 \mathrm{H}, J=7.5 \mathrm{~Hz})$, $7.56(\mathrm{t}, 2 \mathrm{H}, J=8.0 \mathrm{~Hz}), 7.68-7.28(\mathrm{~m}, 3 \mathrm{H}), 7.24-7.12(\mathrm{~m}, 2 \mathrm{H}), 7.05(\mathrm{~d}, 1 \mathrm{H}, J=8.8 \mathrm{~Hz})$, $6.97(\mathrm{~d}, 1 \mathrm{H}, J=7.2 \mathrm{~Hz}), 6.88(\mathrm{t}, 2 \mathrm{H}, J=7.1 \mathrm{~Hz}), 6.75(\mathrm{t}, 2 \mathrm{H}, J=8.9 \mathrm{~Hz}), 6.61(\mathrm{~d}, 1 \mathrm{H}, J=$ $8.9 \mathrm{~Hz}), 3.93-3.30(\mathrm{~m}, 4 \mathrm{H}), 1.80-1.58(\mathrm{~m}, 2 \mathrm{H}), 1.40-1.10(\mathrm{~m}, 16 \mathrm{H}), 1.04-0.71(\mathrm{~m}, 12 \mathrm{H})$; ${ }^{13} \mathrm{C}$ NMR $\left(\mathrm{CDCl}_{3}, 76 \mathrm{MHz}\right) \delta_{\mathrm{C}} 162.8,162.0,137.3,132.8,131.0,129.2,128.4,125.4$, 123.6, 122.3, 120.7, 100.7, 97.2, 44.0, 38.0, 30.9, 28.7, 24.1, 23.3, 14.3, 10.7. HRMS (APCI+) m/z: [M] ${ }^{+}$calcd for $\mathrm{C}_{58} \mathrm{H}_{50} \mathrm{~N}_{2} \mathrm{O}_{4}, 838.3765$; found, 838.3788.

\section{Boron, difluoro[3-iodo-5-[1-(4-iodo-3,5-dimethyl-2H-pyrrol-2-ylidene- $\mathrm{kN})$ hexadecyl] -2,4-dimethyl-1H-pyrrolato-kN]}

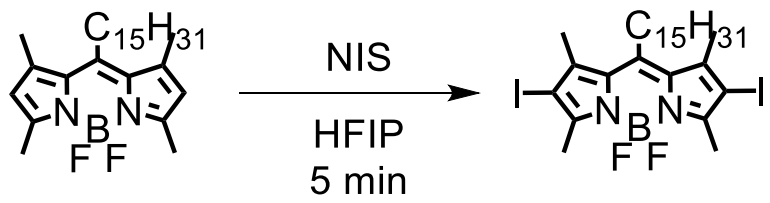

Boron, difluoro[3-iodo-5-[1-(4-iodo-3,5-dimethyl-2H-pyrrol-2-ylidene- $\mathrm{kN})$ pentadecyl]2,4-dimethyl-1H-pyrrolato- $\mathrm{kN}$ ] was prepared adapting a literature procedure of a hexafluoropropanol assisted iodation of BODIPYs ${ }^{20}$. It was synthesized from boron, [2-[1-(3, 5-dimethyl-2H-pyrrol-2-ylidene- $\mathrm{kN}$ )hexadecyl]-3,5-dimethyl-1H-pyrrolato-kN]difluoro (100 mg, $0.22 \mathrm{mmol}$ ) in $83 \%$ yield ( $130 \mathrm{mg}, 0.183 \mathrm{mmol}$ ) as a bright orange solid. ${ }^{1} \mathrm{H}$ NMR $\left(\mathrm{CDCl}_{3}, 400 \mathrm{MHz}\right): \delta_{\mathrm{H}} 3.02-2.88(\mathrm{~m}, 2 \mathrm{H}), 2.61(\mathrm{~s}, 6 \mathrm{H}), 2.46(\mathrm{~s}, 6 \mathrm{H}), 1.66-1.53(\mathrm{~m}, 2 \mathrm{H})$, $1.53-1.40(\mathrm{~m}, 2 \mathrm{H}), 1.39-1.11(\mathrm{~m}, 22 \mathrm{H}), 0.88(\mathrm{t}, 2 \mathrm{H}, J=6.8 \mathrm{~Hz}) ;{ }^{13} \mathrm{C}$ NMR $\left(\mathrm{CDCl}_{3}, 101\right.$ MHz) $\delta_{\mathrm{C}} 155.3,146.6,142.4,131.5,86.5,53.6,32.1,31.8,30.4,29.8,29.8,29.8,29.8$, 29.7, 29.7, 29.5, 29.4, 22.8, 19.1, 19.0, 16.3, 16.2, 16.2, 14.3; HRMS (ESI+) m/z: [M+Na] ${ }^{+}$ calcd for $\mathrm{C}_{28} \mathrm{H}_{43} \mathrm{BF}_{2} \mathrm{I}_{2} \mathrm{~N}_{2} \mathrm{Na}$, 733.1474; found, 733.1472.

\section{PyrBPY}

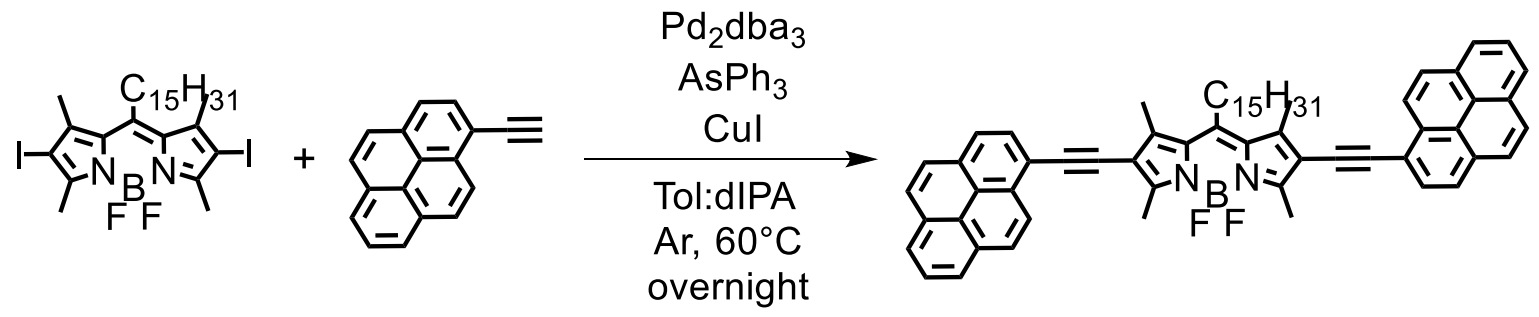


2PyrBPY was synthesized using a Sonogashira under Glaser-Hay free coupling conditions already reported by our group ${ }^{19}$. Boron, difluoro[3-iodo-5-[1-(4-iodo-3,5-dimethyl-2Hpyrrol-2-ylidene- $\mathrm{kN}$ )hexadecyl]-2,4-dimethyl-1H-pyrrolato- $\mathrm{kN}$ ] ( $84 \mathrm{mg}, 0.118 \mathrm{mmol})$ and ethynyl pyrene $(64 \mathrm{mg}, 0.281 \mathrm{mmol})$ were dissolved in a degassed, and argon protected solution of toluene $(287 \mathrm{~mL})$ and diisopropylamine $(8.9 \mathrm{~mL})$. The temperature was adjusted to $60^{\circ} \mathrm{C}$ and $\mathrm{Pd}_{2} \mathrm{dba}_{3}(22 \mathrm{mg}, 0.0237 \mathrm{mmol})$ and $\mathrm{AsPh}_{3}(109 \mathrm{mg}, 0.355 \mathrm{mmol})$ were directly added as solid. A $1 \mathrm{~mL}$ syringe was filled with catalysts, purged three times with argon, poured in the solution, and fill in and out repeatedly until all catalysts were transferred to the solution. The solution was stirred overnight. The mixture was washed with water $(3 \times 100 \mathrm{~mL})$, extracted with $\mathrm{CH}_{2} \mathrm{Cl}_{2}(3 \times 100 \mathrm{~mL})$, dried on $\mathrm{MgSO}_{4}$ and the solvent was removed under reduced pressure. The crude product was purified by silica column chromatography (8\% of EtOAc in Hexane) followed by an overnight hot methanol washing step using a Soxhlet apparatus to yield $16 \%(17 \mathrm{mg}, 0.019 \mathrm{mmol})$ of 2PyrBPY as a blue solid. ${ }^{1} \mathrm{H}$ NMR $\left(\mathrm{CDCl}_{3}, 300 \mathrm{MHz}\right): \delta_{\mathrm{H}} 8.62(\mathrm{~d}, 2 \mathrm{H}, J=9.1 \mathrm{~Hz}), 8.28-7.99(\mathrm{~m}, 16 \mathrm{H})$, 3.09-2.96 (m, 2H), 2.91 (s, 6H), $2.72(\mathrm{~s}, 6 \mathrm{H}), 1.78-1.63(\mathrm{~m}, 2 \mathrm{H}), 1.70-1.48(\mathrm{~m}, 24 \mathrm{H}), 1.02-$ $0.77(\mathrm{~m}, 3 \mathrm{H}) ;{ }^{13} \mathrm{C} \mathrm{NMR}\left(\mathrm{CDCl}_{3}, 101 \mathrm{MHz}\right) \delta_{\mathrm{C}} 131.5,131.4,131.2,131.2,129.58,128.6$, 128.2, 127.4, 126.4, 125.7, 125.5, 124.7, 124.5, 118.2, 96.1, 87.9, 32.1, 32.0, 29.9, 29.8, 29.8, 29.6, 29.5, 22.8, 15.6, 14.3, 14.1. HRMS (ESI+) m/z: [M] calcd for $\mathrm{C}_{64} \mathrm{H}_{61} \mathrm{BF}_{2} \mathrm{~N}_{2}$, 906.4901; found, 906.4903 . 


\section{Synthesis of GNR}
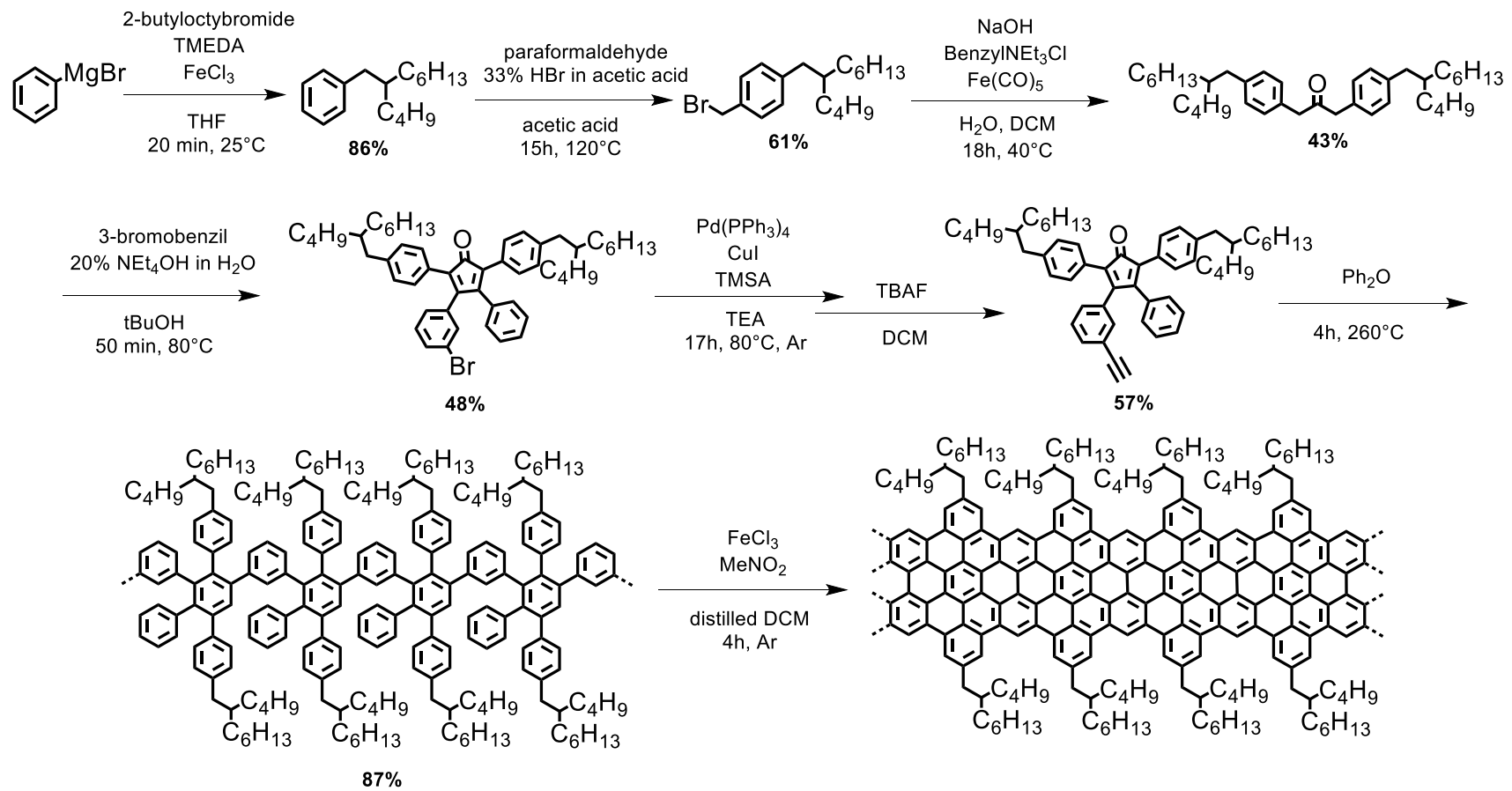

\section{2-Butyloctylbenzene}

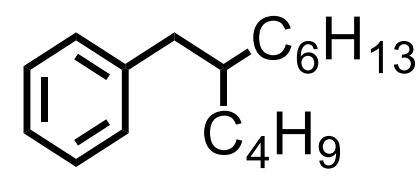

2-Butyloctylbenzene was synthesized from an adapted literature procedure of an iron catalyzed Grignard coupling method ${ }^{21}$ and was synthesized from 2-butyloctyl bromide $(49.8 \mathrm{~g}, 200 \mathrm{mmol})$ in $86 \%$ yields $(42.8 \mathrm{~g}, 174 \mathrm{mmol})$ as a colorless oil. ${ }^{1} \mathrm{H} \mathrm{NMR}\left(\mathrm{CDCl}_{3}\right.$, $300 \mathrm{MHz}): \delta_{\mathrm{H}} 7.41-7.11(\mathrm{~m}, 5 \mathrm{H}), 2.60(\mathrm{~d}, 2 \mathrm{H}, J=7.0 \mathrm{~Hz}), 1.82-1.55(\mathrm{~m}, 1 \mathrm{H}), 1.55-1.12$ $(\mathrm{m}, 16 \mathrm{H}), 0.95(\mathrm{t}, 6 \mathrm{H}, J=5.9 \mathrm{~Hz}) ;{ }^{13} \mathrm{C} \mathrm{NMR}\left(\mathrm{CDCl}_{3}, 101 \mathrm{MHz}\right) ; \delta_{\mathrm{C}} 142.0,129.3,128.2$, 125.6, 40.7, 39.8, 33.3, 33.0, 32.1, 29.8, 29.0, 26.7, 23.2, 22.8, 14.3, 14.3; HRMS (APCI+) $\mathrm{m} / \mathrm{z}$ : $[\mathrm{M}]^{+}$calcd for $\mathrm{C}_{18} \mathrm{H}_{30}, 246.2342$; found, 246.2348 .

\section{1-(Bromomethyl)-4-(2-butyloctyl)benzene}

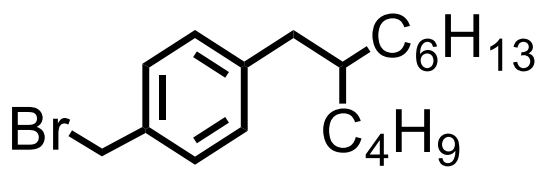

1-(Bromomethyl)-4-(2-butyloctyl)benzene was prepared using an adapted procedure from the literature ${ }^{22}$ and was synthesized from 2-butyloctylbenzene (20 g, $\left.82.1 \mathrm{mmol}\right)$ in $61 \%$ yield $(17 \mathrm{~g}, 50 \mathrm{mmol})$ as a colorless oil. Both the main product (85\%) and a geometric isomer $(15 \%)$ were present and failed to separate. The mixture was used as such in the next 
step of the synthesis. ${ }^{1} \mathrm{H}$ NMR $\left(\mathrm{CDCl}_{3}, 300 \mathrm{MHz}\right): \delta_{\mathrm{H}} 7.35(\mathrm{~d}, 2 \mathrm{H}, J=8.0 \mathrm{~Hz}), 7.17(\mathrm{~d}, 2 \mathrm{H}$, $J=8.0 \mathrm{~Hz}), 4.54(\mathrm{~s}, 2 \mathrm{H}), 2.58(\mathrm{~d}, 2 \mathrm{H}, J=7.0 \mathrm{~Hz}), 1.78-1.58(\mathrm{~m}, 1 \mathrm{H}), 1.50-1.20(\mathrm{~m}, 16 \mathrm{H})$, 1.03-0.82 (m, 6H); ${ }^{13} \mathrm{C} \mathrm{NMR}\left(\mathrm{CDCl}_{3}, 101 \mathrm{MHz}\right) \delta_{\mathrm{C}} 142.6,135.0,129.7,129.0,40.4,39.7$, 34.0, 33.3, 33.0, 32.0, 29.8, 28.9, 26.7, 23.18, 22.8, 14.3, 14.3; HRMS (ESI+) m/z: $[\mathrm{M}+\mathrm{Na}]^{+}$calcd for $\mathrm{C}_{19} \mathrm{H}_{31} \mathrm{Na}, 361.1501$; found, 361.1504 .

\section{1,3-Bis(4-(2-butyloctyl))propan-2-one}

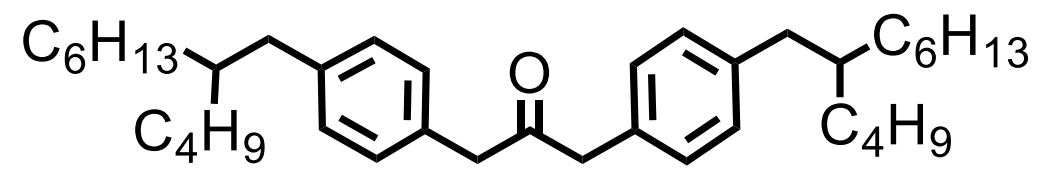

1,3-Bis(4-(2-butyloctyl))propan-2-one was prepared using an adapted procedure from the literature $^{22}$. It was synthesized from 1-(bromomethyl)-4-(2-butyloctyl)benzene (7.0 g, 19.3 $\mathrm{mmol})$ in $43 \%$ yield $(2.47 \mathrm{~g}, 4.17 \mathrm{mmol})$ as a white oily solid. Both the main product (89\%) and a geometric isomer (11\%) were present and failed to separate. The mixture was used as such in the next step of the synthesis. ${ }^{1} \mathrm{H}$ NMR $\left(\mathrm{CDCl}_{3}, 300 \mathrm{MHz}\right): \delta_{\mathrm{H}} 7.21-6.89(\mathrm{~m}$, $8 \mathrm{H}), 3.68(\mathrm{~s}, 4 \mathrm{H}), 2.52(\mathrm{~d}, 4 \mathrm{H}, J=7.0 \mathrm{~Hz}), 1.82-1.41(\mathrm{~m}, 2 \mathrm{H}), 1.38-1.11(\mathrm{~m}, 32 \mathrm{H}), 0.89(\mathrm{t}$, $12 \mathrm{H}, J=6.5 \mathrm{~Hz}) ;{ }^{13} \mathrm{C} \mathrm{NMR}\left(\mathrm{CDCl}_{3}, 76 \mathrm{MHz}\right) \delta_{\mathrm{C}} 206.4,140.8,131.3,129.6,129.3,129.3$, 48.8, 40.3, 39.7, 33.3, 33.0, 32.1, 29.8, 28.9, 26.7, 23.2, 22.8, 14.3, 14.3; HRMS (ESI+) $\mathrm{m} / \mathrm{z}:[\mathrm{M}+\mathrm{Na}]^{+}$calcd for $\mathrm{C}_{39} \mathrm{H}_{62} \mathrm{NaO}, 569.4692$; found, 569.4694 .

\section{3-(3-Bromophenyl)-2,5-bis(4-(2-butyloctyl)phenyl)-4-phenylcyclopenta- 2,4-dienone}

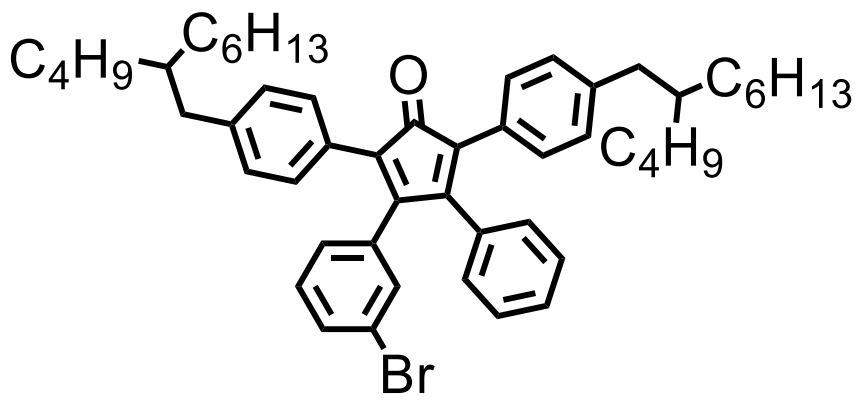

3-(3-Bromophenyl)-2,5-bis(4-(2-butyloctyl)phenyl)-4-phenylcyclopenta-2,4-dienone was prepared using an adapted procedure from the literature ${ }^{22}$. It was synthesized from 1,3bis(4-(2-butyloctyl))propan-2-one (1.32 g, $4.55 \mathrm{mmol})$ and 3-bromobenzil (2.48 g, 4.53 $\mathrm{mmol})$ in $48 \%$ yield $(1.75 \mathrm{~g}, 2.19 \mathrm{mmol})$ as a purple viscous oil. The undesired isomers were removed at this step of the reaction by the already reported purification method. ${ }^{1} \mathrm{H}$ NMR $\left(\mathrm{CDCl}_{3}, 300 \mathrm{MHz}\right): \delta_{\mathrm{H}} 7.51-6.80(\mathrm{~m}, 17 \mathrm{H}), 2.52(\mathrm{t}, 4 \mathrm{H}, J=7.2 \mathrm{~Hz}), 1.74-1.58(\mathrm{~m}$, $2 \mathrm{H}), 1.50-1.14(\mathrm{~m}, 32 \mathrm{H}), 0.92(\mathrm{t}, 12 \mathrm{H}, J=6.9 \mathrm{~Hz}) ;{ }^{13} \mathrm{C} \mathrm{NMR}\left(\mathrm{CDCl}_{3}, 76 \mathrm{MHz}\right) \delta_{\mathrm{C}} 200.7$, $153.7,152.0,141.9,141.6,135.7,133.3,132.4$, 131.4, 129.9, 129.6, 129.4, 129.2, 129.1, $128.7,128.2$, 128.1 127.9, 127.6, 126.0, 125.2, 122.1, 40.6, 39.7, 39.7, 33.4, 33.4, 33.0, 33.0, 32.1, 32.0, 29.8, 29.8, 29.0, 26.7, 23.2, 23.1, 22.8, 14. 3, 14.3; HRMS (ESI+) m/z: $[\mathrm{M}+\mathrm{Na}]^{+}$calcd for $\mathrm{C}_{53} \mathrm{H}_{67} \mathrm{BrNaO}, 823.4260$; found, 823.4266. 


\section{3-(3-Ethynylphenyl)-2,5-bis(4-(2-butyloctyl)phenyl)-4-phenylcyclopenta-2,4-dienone}

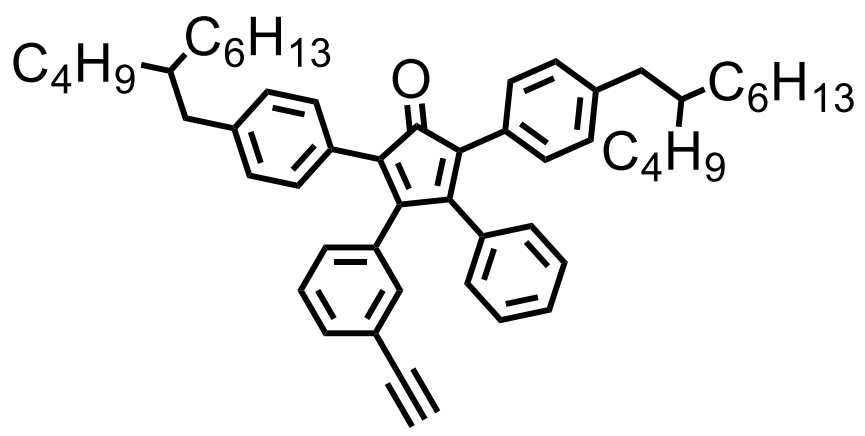

3-(3-Ethynylphenyl)-2,5-bis(4-(2-butyloctyl)phenyl)-4-phenylcyclopenta-2,4-dienone was prepared using an adapted procedure from the literature ${ }^{22}$. It was synthesized from 3(3-bromophenyl)-2,5-bis(4-(2-butyloctyl)phenyl)-4-phenylcyclopenta-2,4-dienone (1.14 $\mathrm{g}, 1.43 \mathrm{mmol})$ in $57 \%$ yield $(610 \mathrm{mg}, 0.82 \mathrm{mmol})$ as a purple viscous oil. ${ }^{1} \mathrm{H} \mathrm{NMR}\left(\mathrm{CDCl}_{3}\right.$, $300 \mathrm{MHz}): \delta_{\mathrm{H}} 7.46-6.75(\mathrm{~m}, 17 \mathrm{H}), 2.96(\mathrm{~s}, 1 \mathrm{H}), 2.59-2.34(\mathrm{~m}, 4 \mathrm{H}), 1.73-1.54(\mathrm{~m}, 2 \mathrm{H})$, 1.41-1.0 (m, 32H), 1.0-0.63 (m, 12H); ${ }^{13} \mathrm{C} \mathrm{NMR}\left(\mathrm{CDCl}_{3}, 76 \mathrm{MHz}\right) \delta_{\mathrm{C}} 201.0,153.8,152.7$, 141.8, 141.6, 134.0, 133.3, 132.9, 132.1, 129.9, 129.4, 129.2, 129.1, 128.6, 128.2, 128.0, 127.7, 125.8, 125.2, 122.1, 83.1, 40.6, 39.7, 33.4, 33.0, 33.0, 32.1, 29.8, 29.0, 26.7, 23.2, 22.8, 14.3; HRMS (ESI+) m/z: [M+Na] ${ }^{+}$calcd for $\mathrm{C}_{55} \mathrm{H}_{68} \mathrm{NaO}, 767.5162$; found, 767.5152.

\section{GNR precursor}

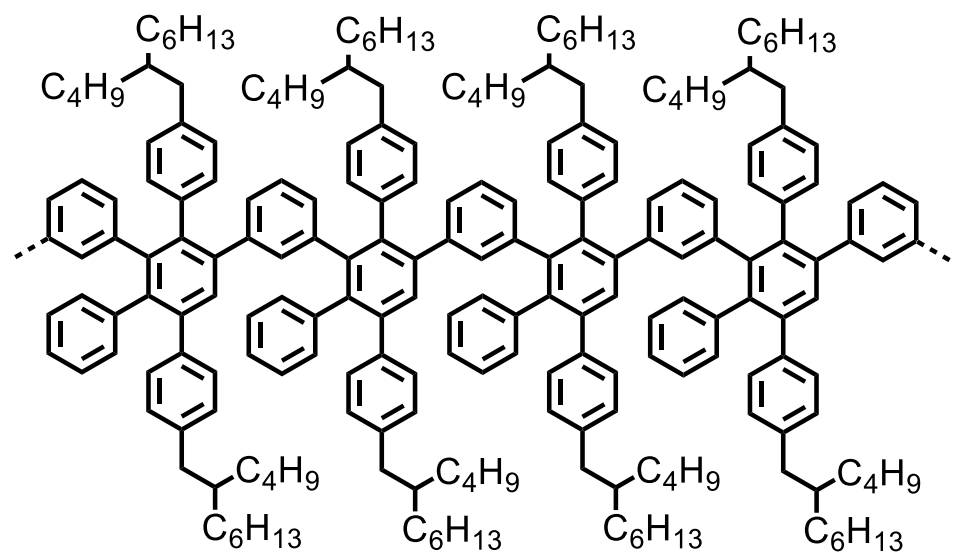

GNR precursor was prepared using an adapted procedure of a Diels-Alder polymerization in $\mathrm{Ph}_{2} \mathrm{O}$ solution from the literature ${ }^{11}$. It was synthesized from 3-(3-ethynylphenyl)-2,5bis(4-(2-butyloctyl)phenyl)-4-phenylcyclopenta-2,4-dienone (48 $\mathrm{mg}, 0.065 \mathrm{mmol})$. The crude polymer was purified by a $24 \mathrm{~h}$ hot $\mathrm{MeOH}$ washing with a Soxhlet apparatus (instead of purification by a preparative SEC system as used in the original work) giving GNR precursor in $87 \%$ yield $(0.057 \mathrm{mmol}, 41 \mathrm{mg})$ as a white solid. GPC (polystyrene calibration) $\mathrm{M}_{\mathrm{n}}: 24.8 \mathrm{~kg} \mathrm{~mol}^{-1}, \mathrm{M}_{\mathrm{w}}: 49.7 \mathrm{~kg} \cdot \mathrm{mol}^{-1}$, PDI: $2.0 ;{ }^{1} \mathrm{H} \mathrm{NMR}\left(\mathrm{CDCl}_{3}, 300 \mathrm{MHz}\right)$ : $\delta_{\mathrm{H}} 7.13-6.21(\mathrm{br} \mathrm{m}, 18 \mathrm{H}), 2.66-2.14(\mathrm{br} \mathrm{d}, 4 \mathrm{H}), 1.67-1.39(\mathrm{~m}, 2 \mathrm{H}), 1.32-0.97(\mathrm{~m}, 32 \mathrm{H})$, $0.92-0.60$ (br s, 12H). 


\section{GNR}

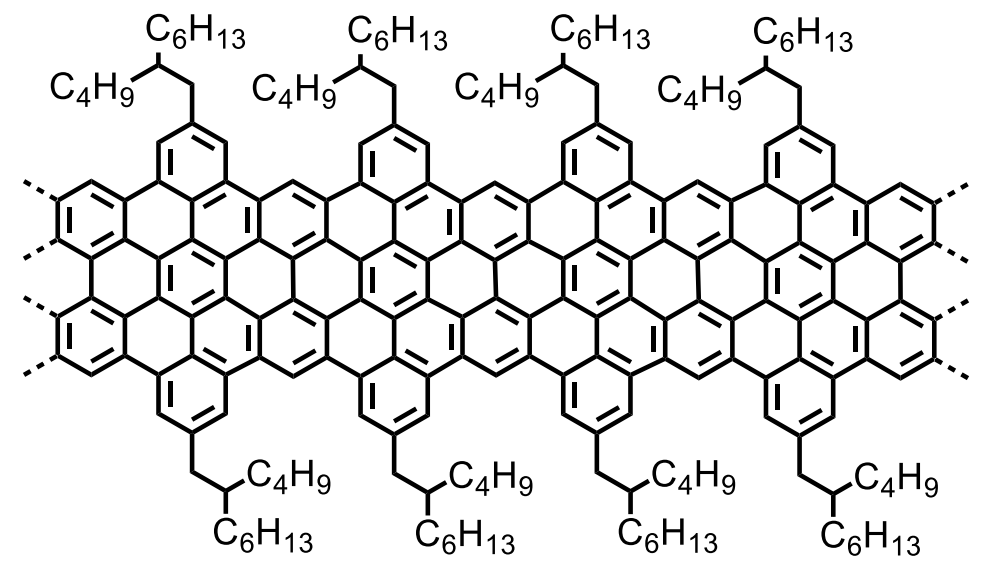

The GNR was prepared according to a modified procedure from the literature ${ }^{11}$. A solution of GNR precursor $(41 \mathrm{mg}, 0.057 \mathrm{mmol})$ in distilled $\mathrm{CH}_{2} \mathrm{Cl}_{2}(215 \mathrm{~mL})$ was degassed by argon bubbling for $10 \mathrm{~min}$. Iron(III) chloride $(790 \mathrm{mg}, 4.87 \mathrm{mmol}$ ) in nitromethane (13 $\mathrm{mL}$ )was added to the reaction mixture. The solution was stirred for $4 \mathrm{~h}$ at room temperature. The reaction was monitored by UV-vis spectroscopy and stirred another hour once the main absorption band at $\sim 580 \mathrm{~nm}$ no longer shifts. The reaction was quenched by $\mathrm{MeOH}$ (400 mL). The red precipitate was filtered with wool, washed generously with $\mathrm{MeOH}$ and recovered with $\mathrm{CHCl}_{3}$. A particular attention was paid to prevent any irreversible drying of the red solid during all the filtration processes. The GNR polymer was stored several months as a deep red GNR solution in chloroform. 


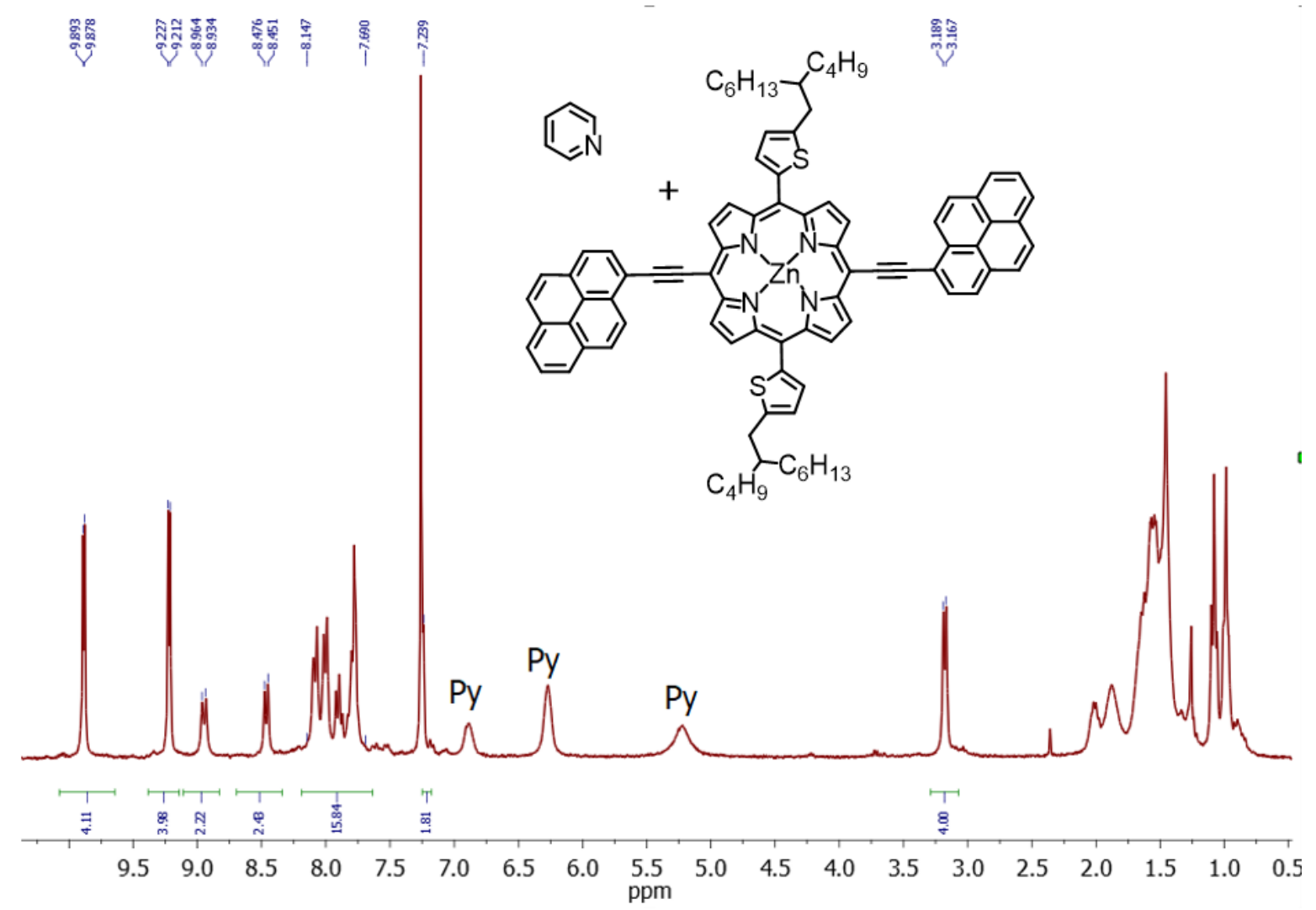

Figure S29. $300 \mathrm{MHz}{ }^{1} \mathrm{H}$ NMR spectrum of $2 \mathrm{PyrPOR}$ in $\mathrm{CDCl}_{3}$

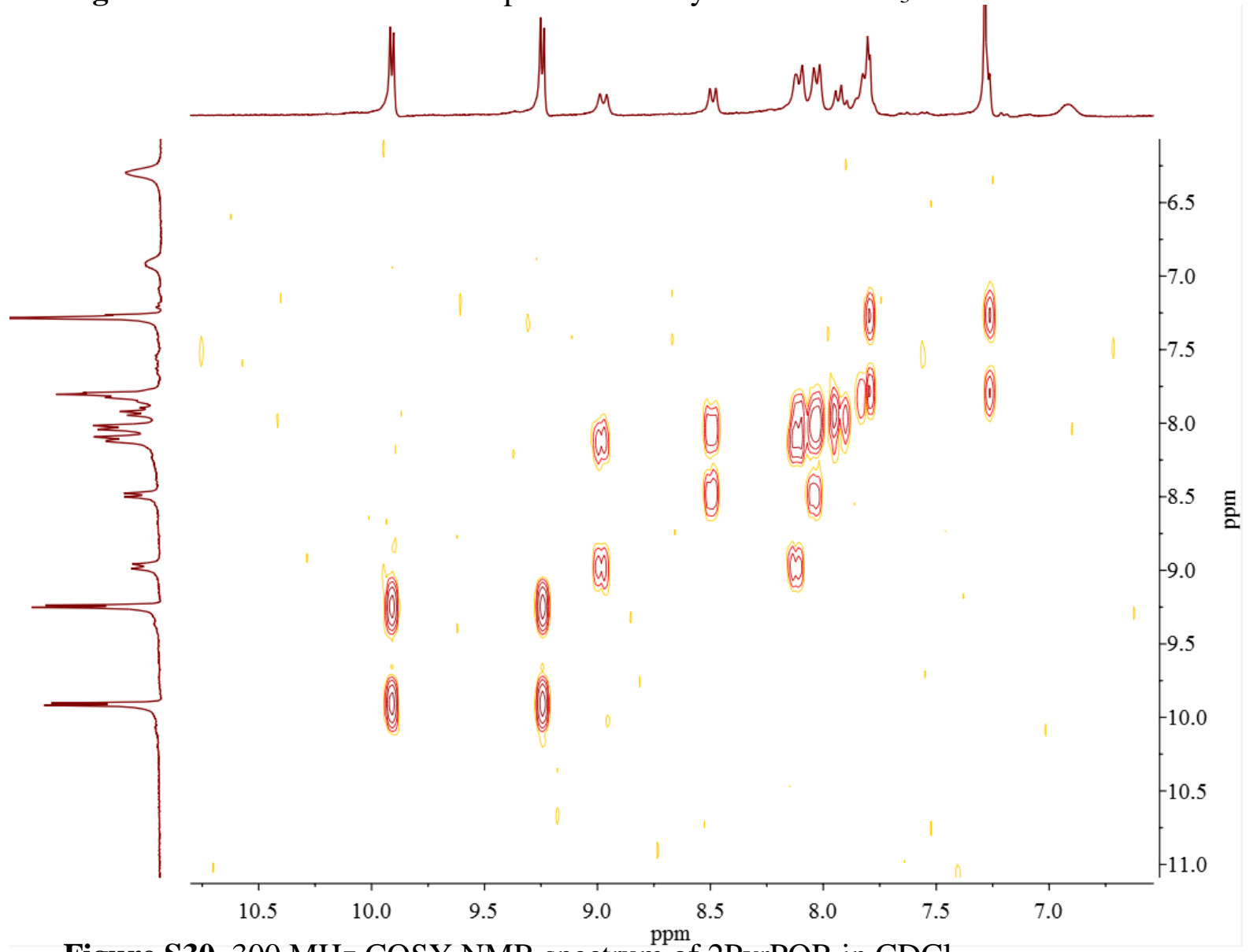

Figure S30. $300 \mathrm{MHz}$ COSY NMR spectrum of $2 \mathrm{PyrPOR}$ in $\mathrm{CDCl}_{3}$

Page S-42 


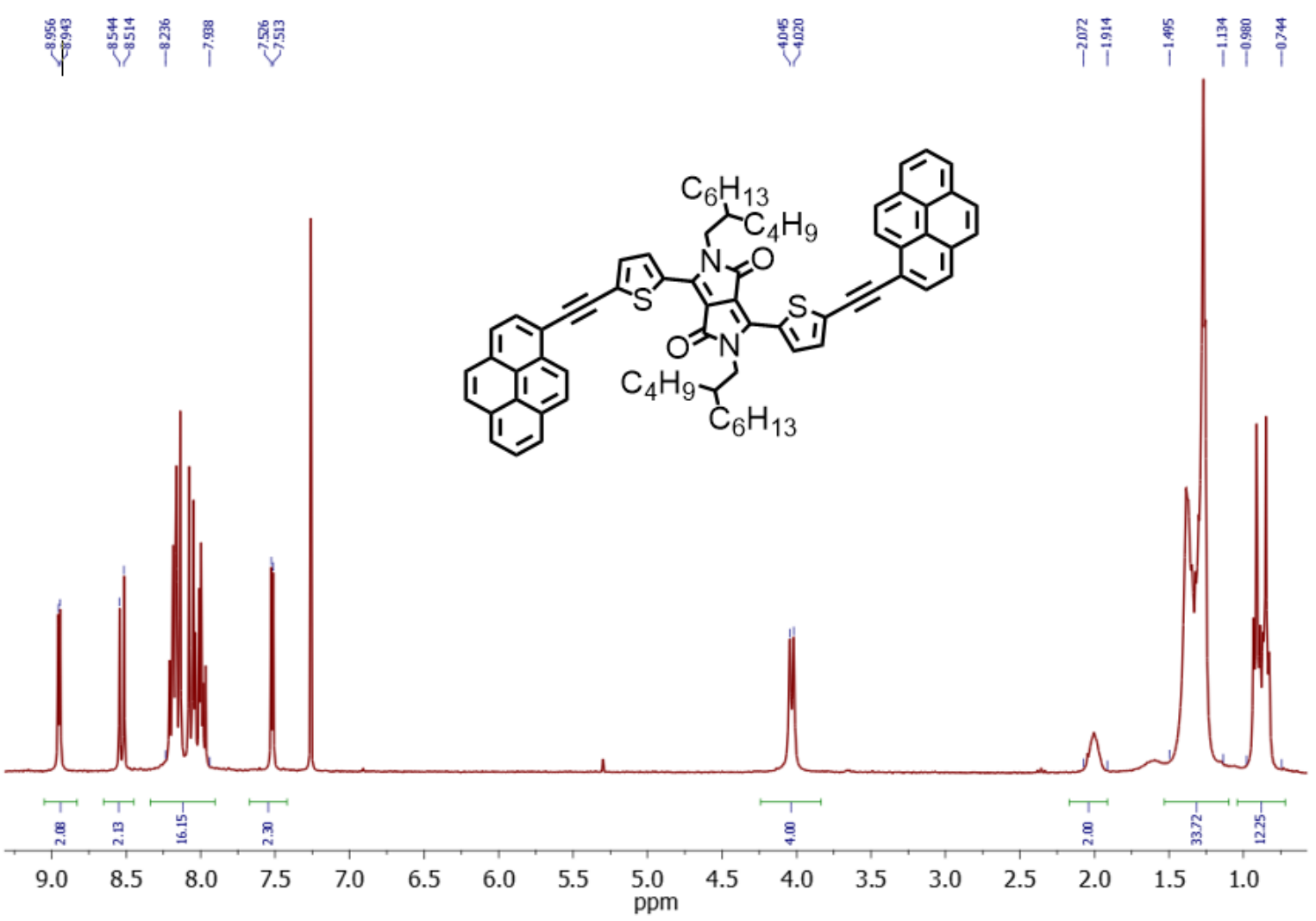

Figure S31. $300 \mathrm{MHz}{ }^{1} \mathrm{H} \mathrm{NMR}$ spectrum of $2 \mathrm{PyrDPP}$ in $\mathrm{CDCl}_{3}$

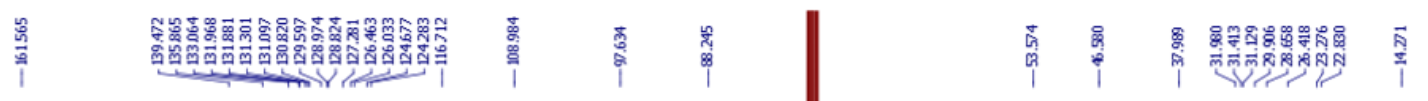

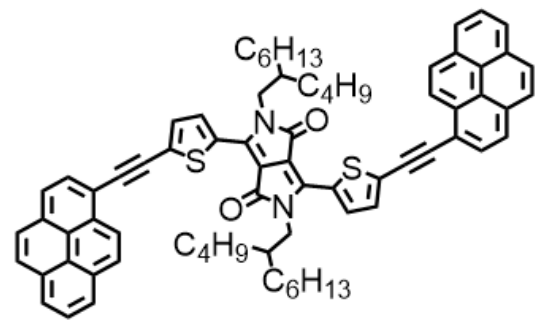

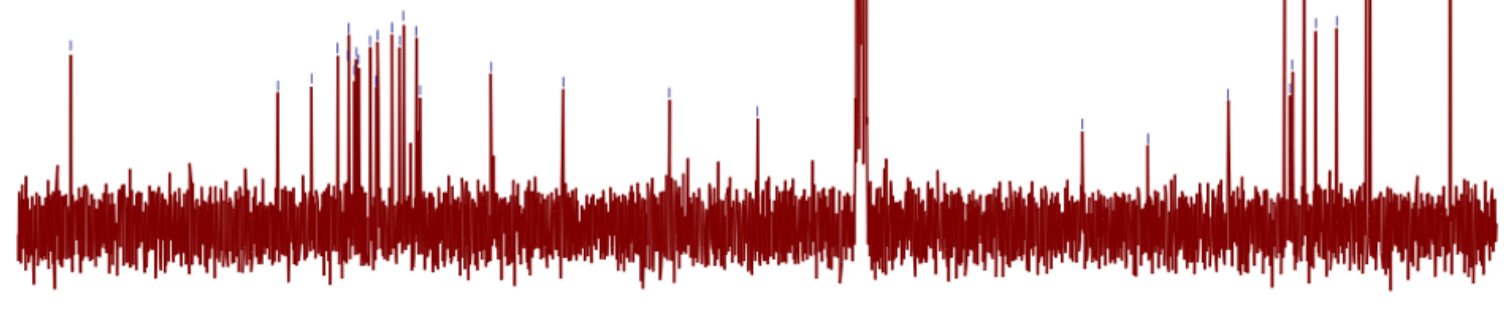

$\begin{array}{llllllllllllllll}160 & 150 & 140 & 130 & 120 & 110 & 100 & \begin{array}{c}90 \\ \mathrm{ppm}\end{array} & 80 & 70 & 60 & 50 & 40 & 30 & 20 & 10\end{array}$

Figure S32. $400 \mathrm{MHz}{ }^{13} \mathrm{C} \mathrm{NMR}$ spectrum of $2 \mathrm{PyrDPP}$ in $\mathrm{CDCl}_{3}$ 


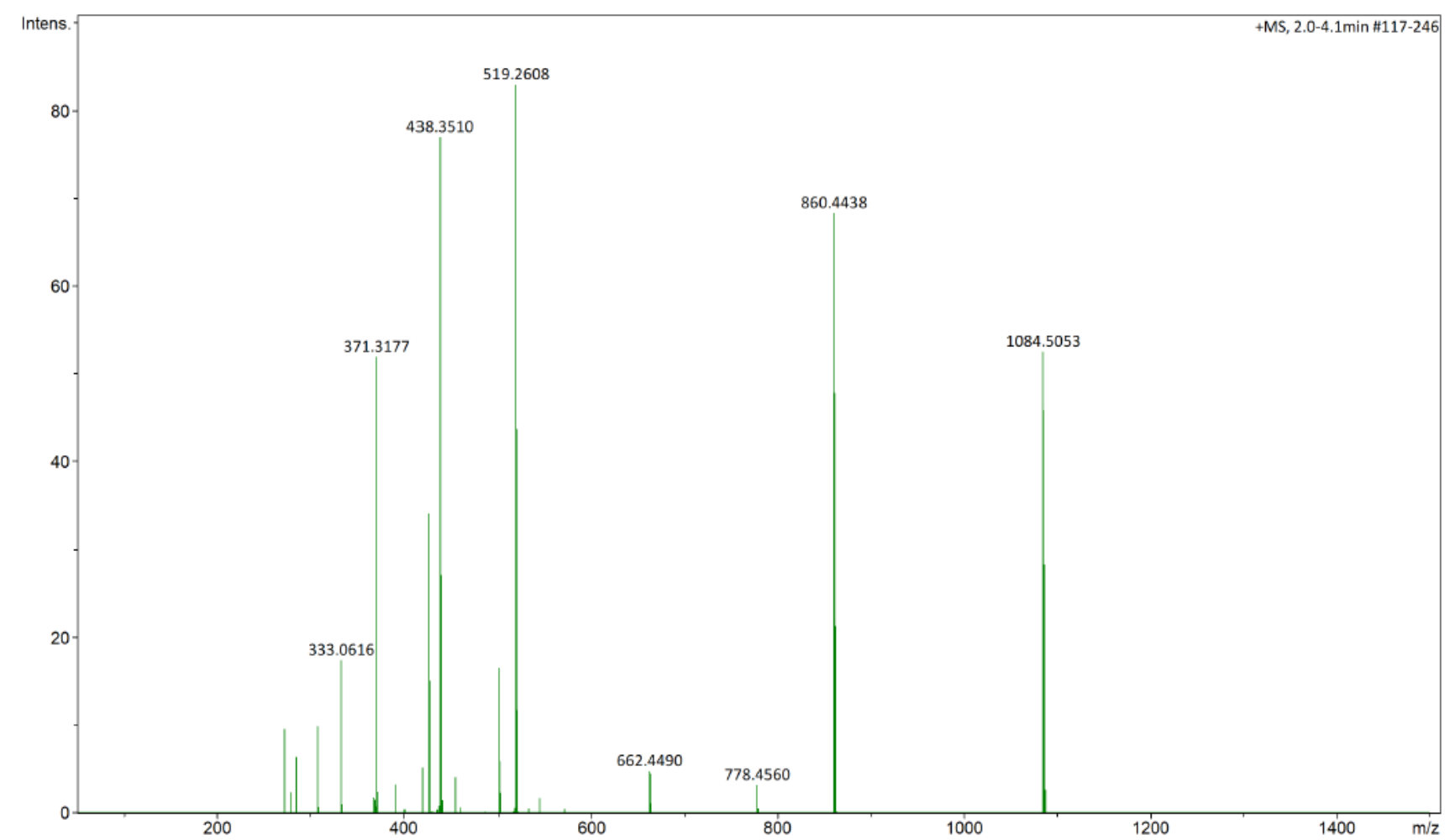

Figure S33. High resolution mass spectrum (APCI+) of 2PyrDPP
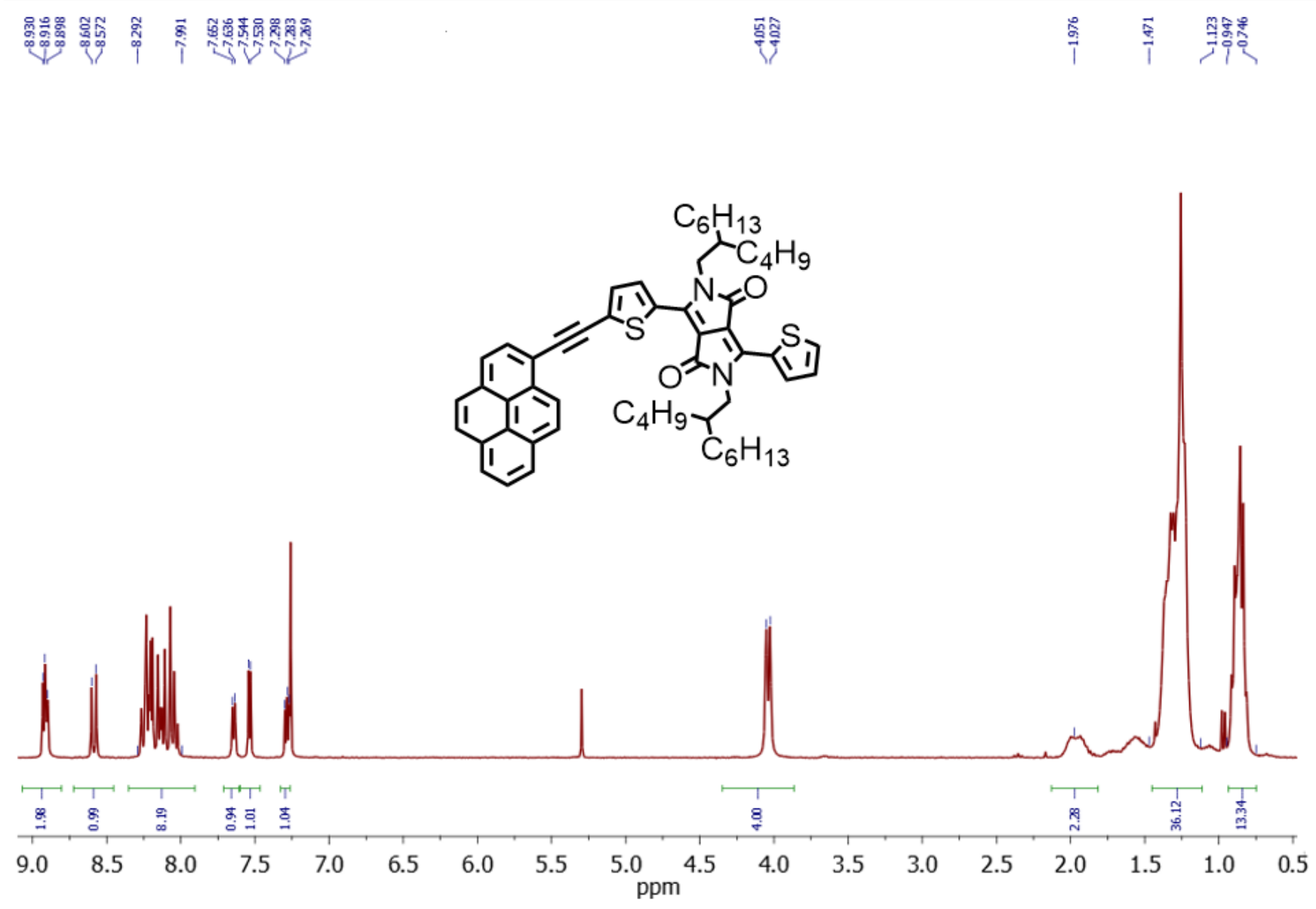

Figure S34. $300 \mathrm{MHz}{ }^{1} \mathrm{H} \mathrm{NMR}$ spectrum of $1 \mathrm{PyrDPP}$ in $\mathrm{CDCl}_{3}$ 


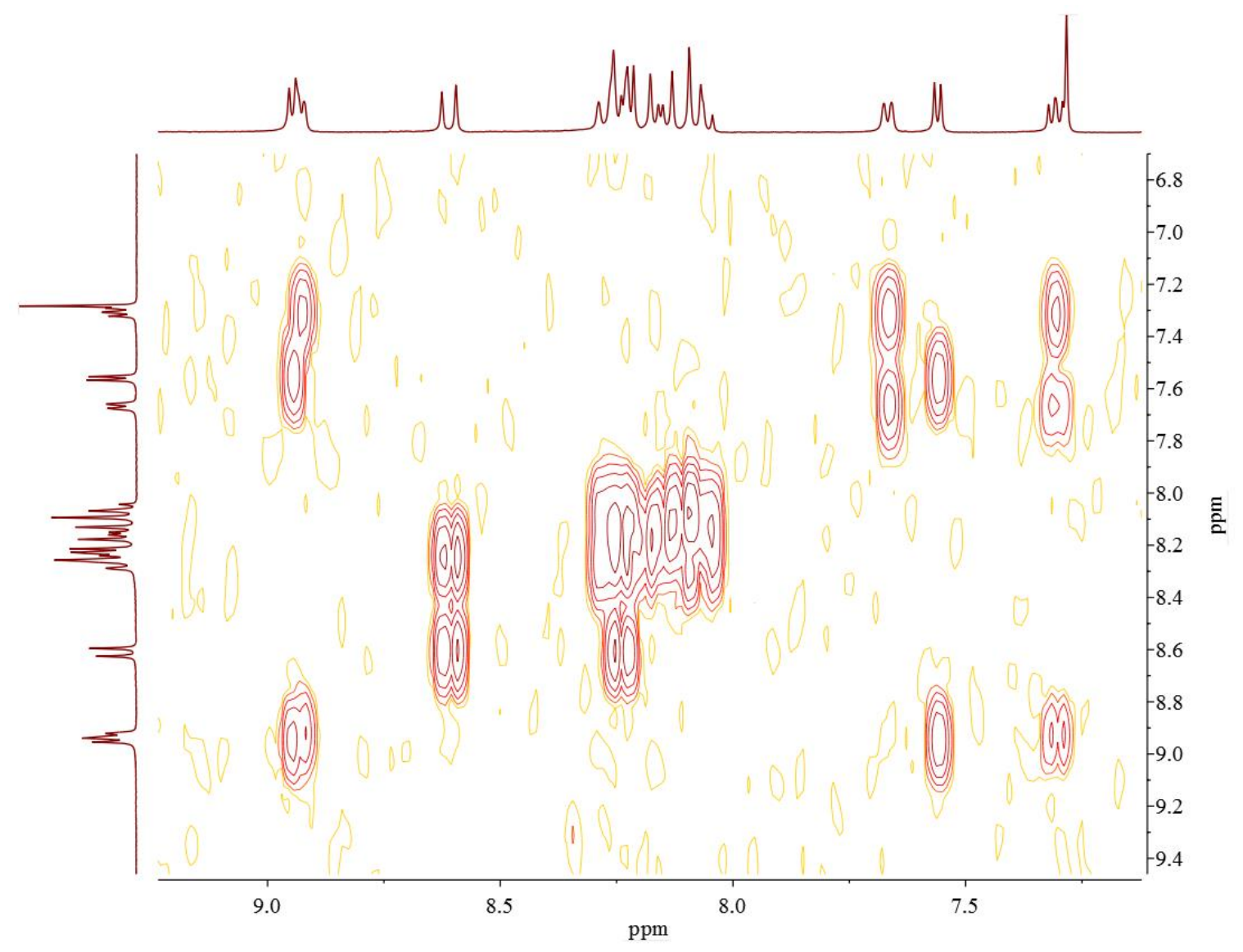

Figure S35. $300 \mathrm{MHz}$ COSY NMR spectrum of 1PyrDPP in $\mathrm{CDCl}_{3}$
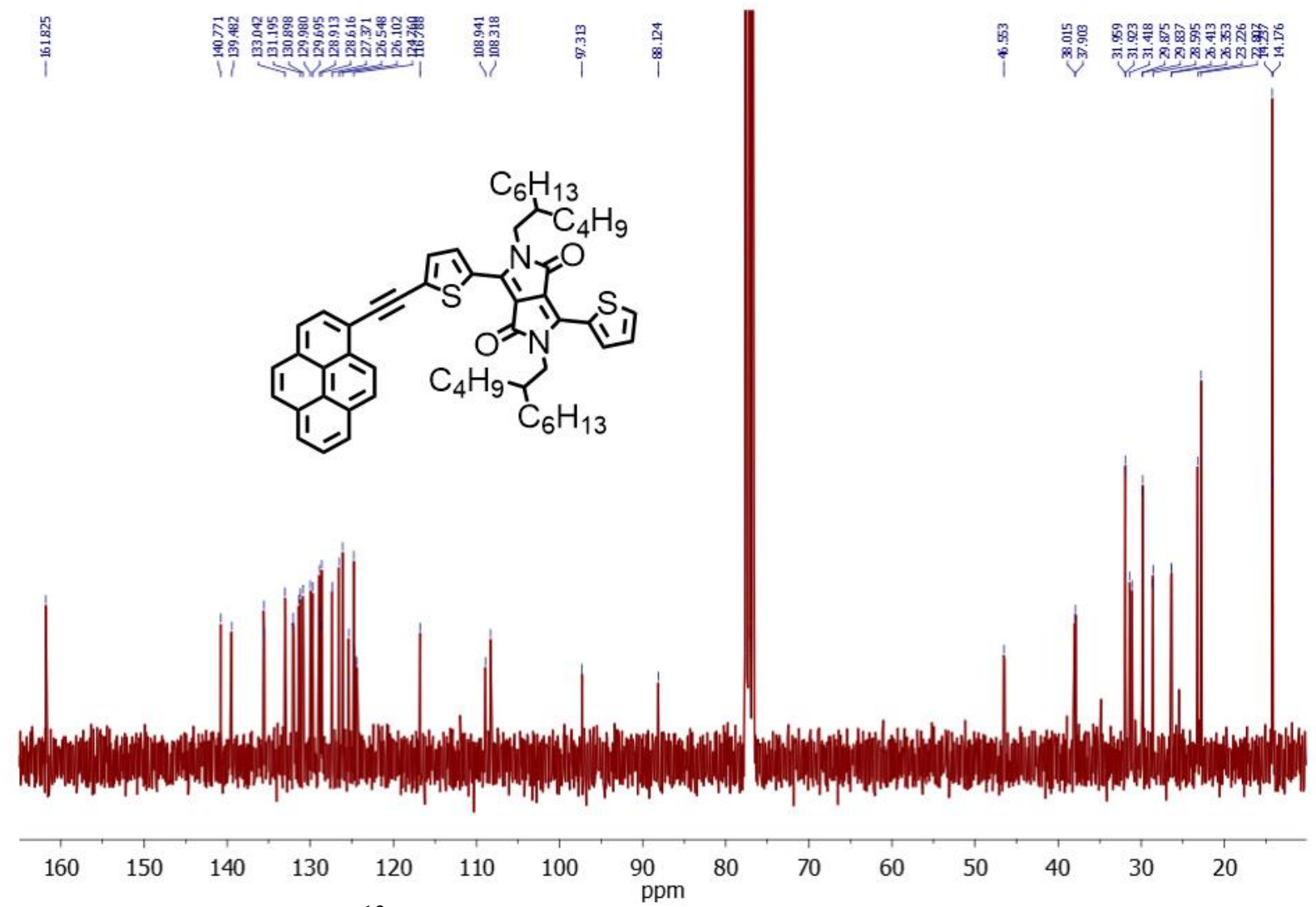

Figure S36. $400 \mathrm{MHz}{ }^{13} \mathrm{C} \mathrm{NMR}$ spectrum of $1 \mathrm{PyrDPP}$ in $\mathrm{CDCl}_{3}$ 


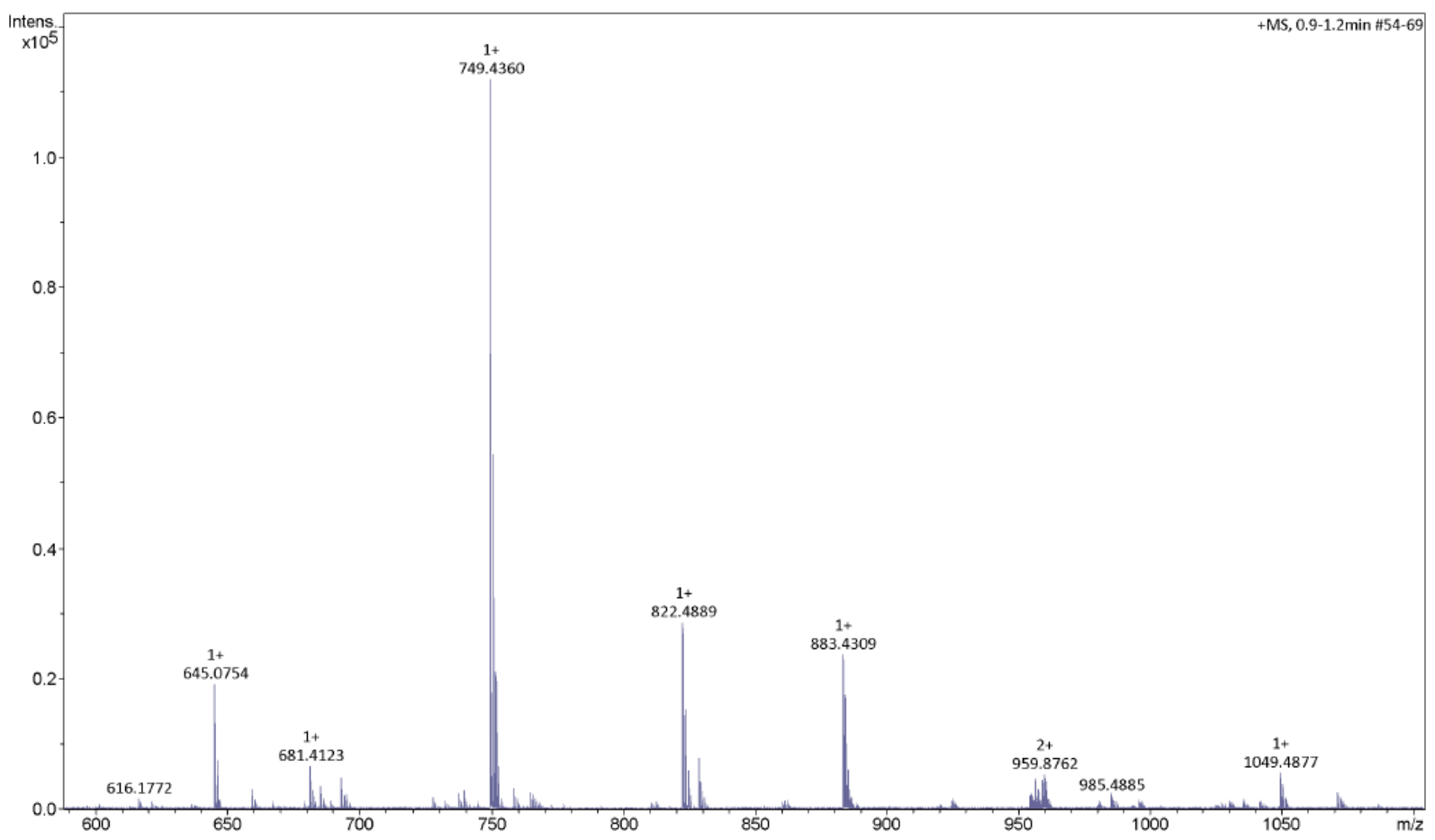

Figure S37. High resolution mass spectrum (ESI+) of 1PrDPP

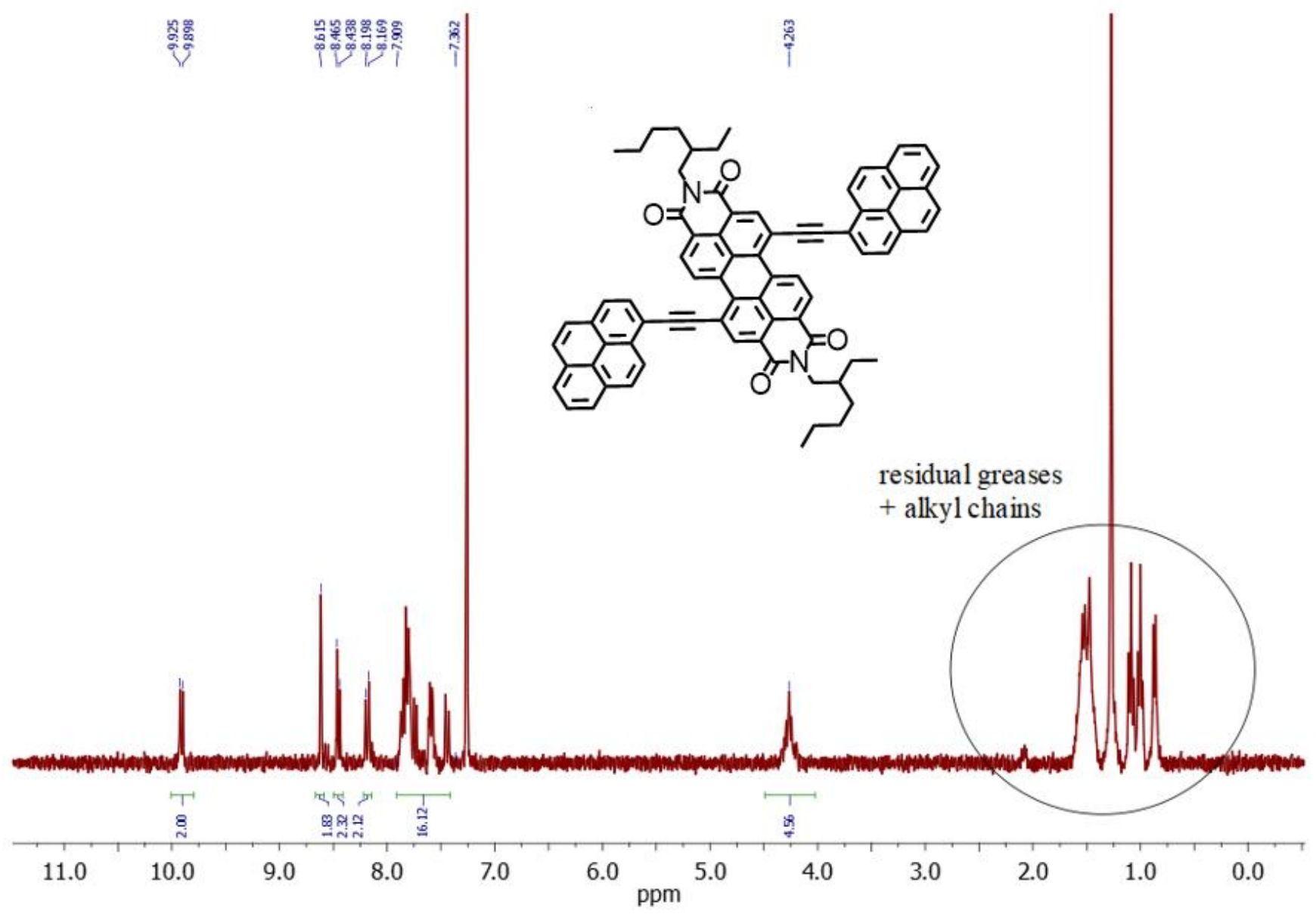

Figure S38. $300 \mathrm{MHz}{ }^{1} \mathrm{H}$ NMR spectrum of $2 \mathrm{PyrPBI}$ in $\mathrm{CDCl}_{3}$ (note: the recrystallisation of 2PyrPBI inside the NMR tube decreases the 2PyrPBI signal and overexpresses the residual greases relatively to the $2 \mathrm{Pyr} \mathrm{PBI}$ signal) 


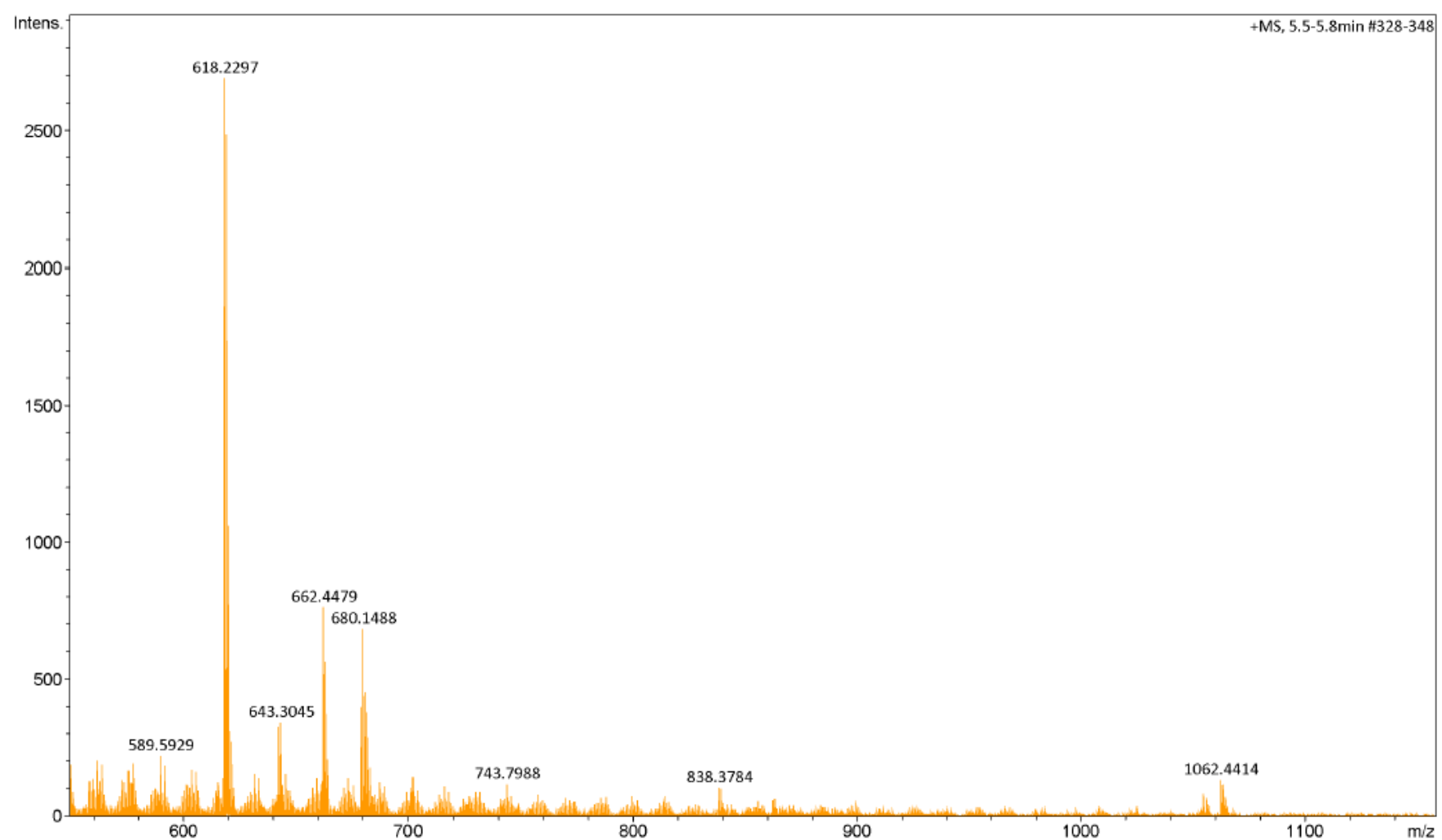

Figure S39. High resolution mass spectrum (APCI+) of 2PyrPBI

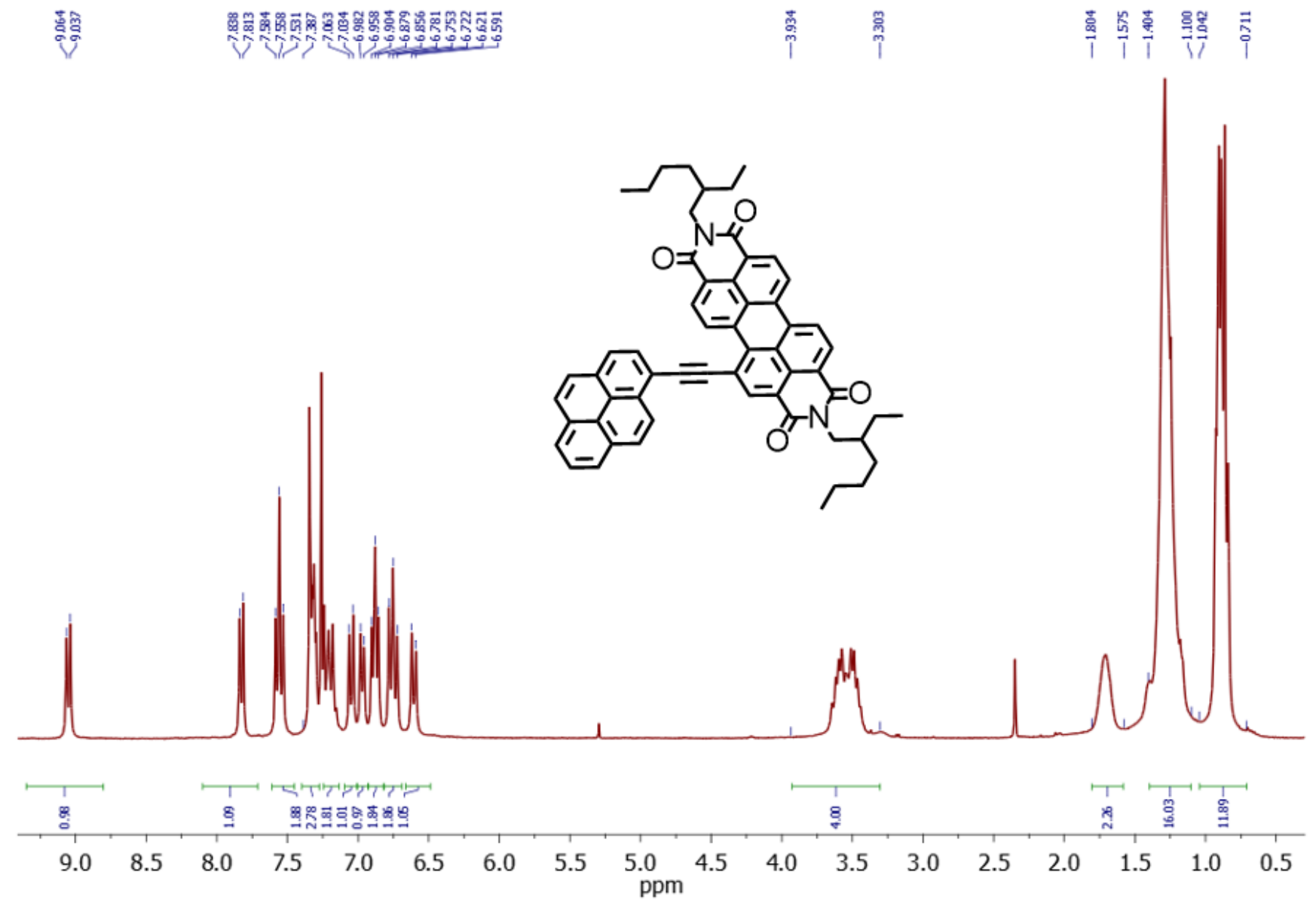

Figure S40. $300 \mathrm{MHz}{ }^{1} \mathrm{H}$ NMR spectrum of $1 \mathrm{PyrPBI}$ in $\mathrm{CDCl}_{3}$ 


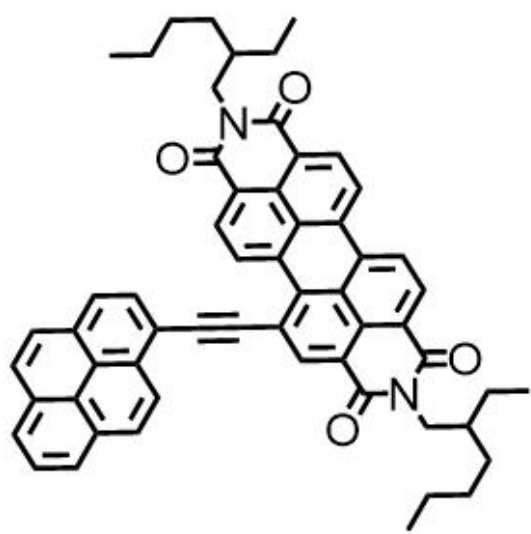

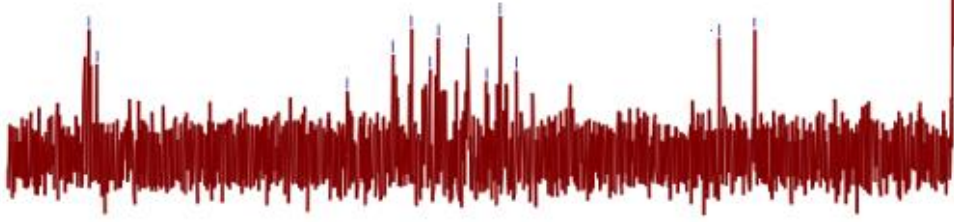

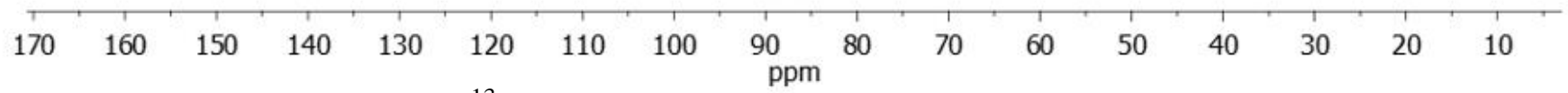

Figure S41. $400 \mathrm{MHz}{ }^{13} \mathrm{C}$ NMR spectrum of $1 \mathrm{PyrPBI}$ in $\mathrm{CDCl}_{3}$

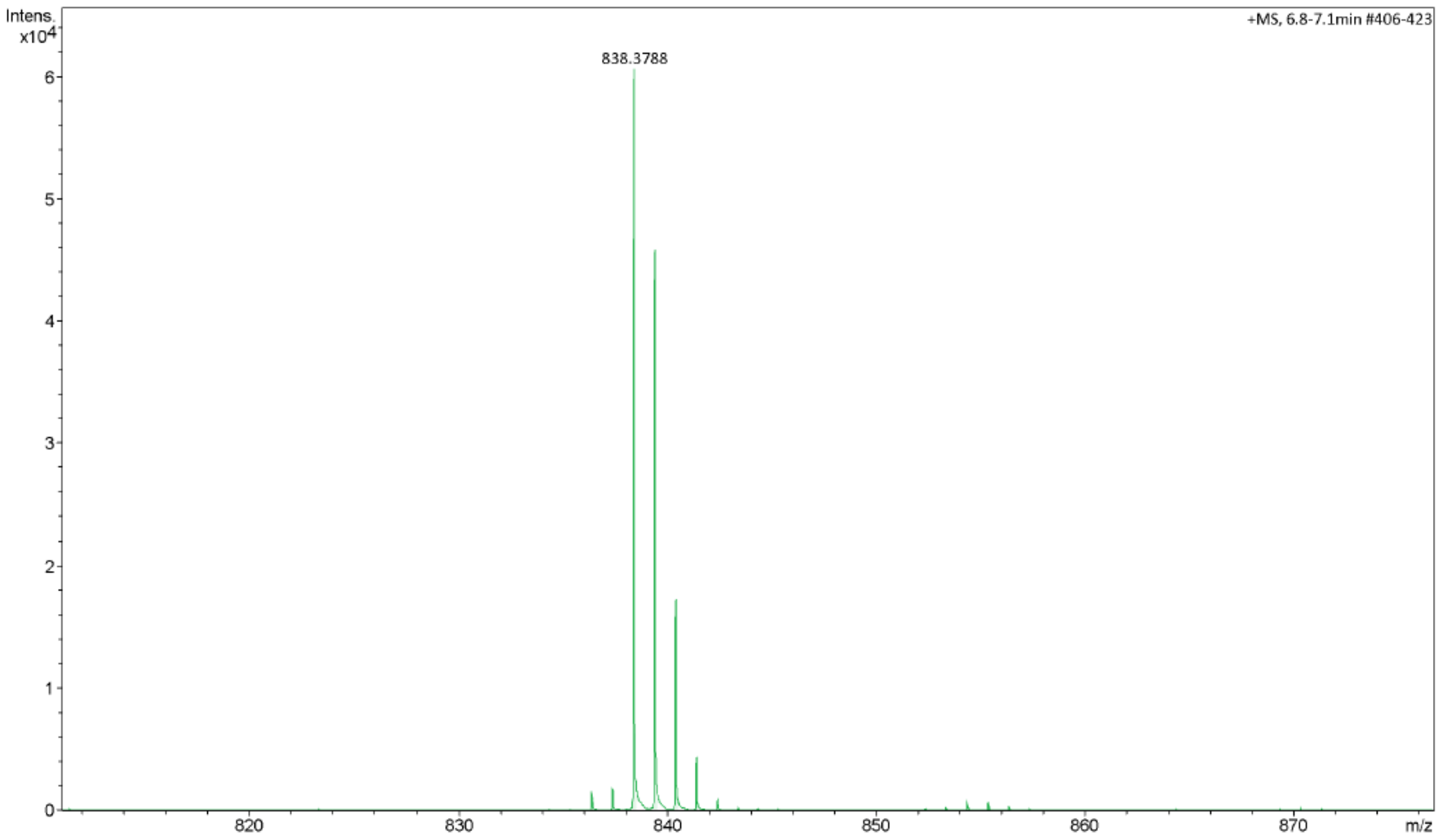

Figure S42. High resolution mass spectrum (APCI+) of 1PyrPBI 

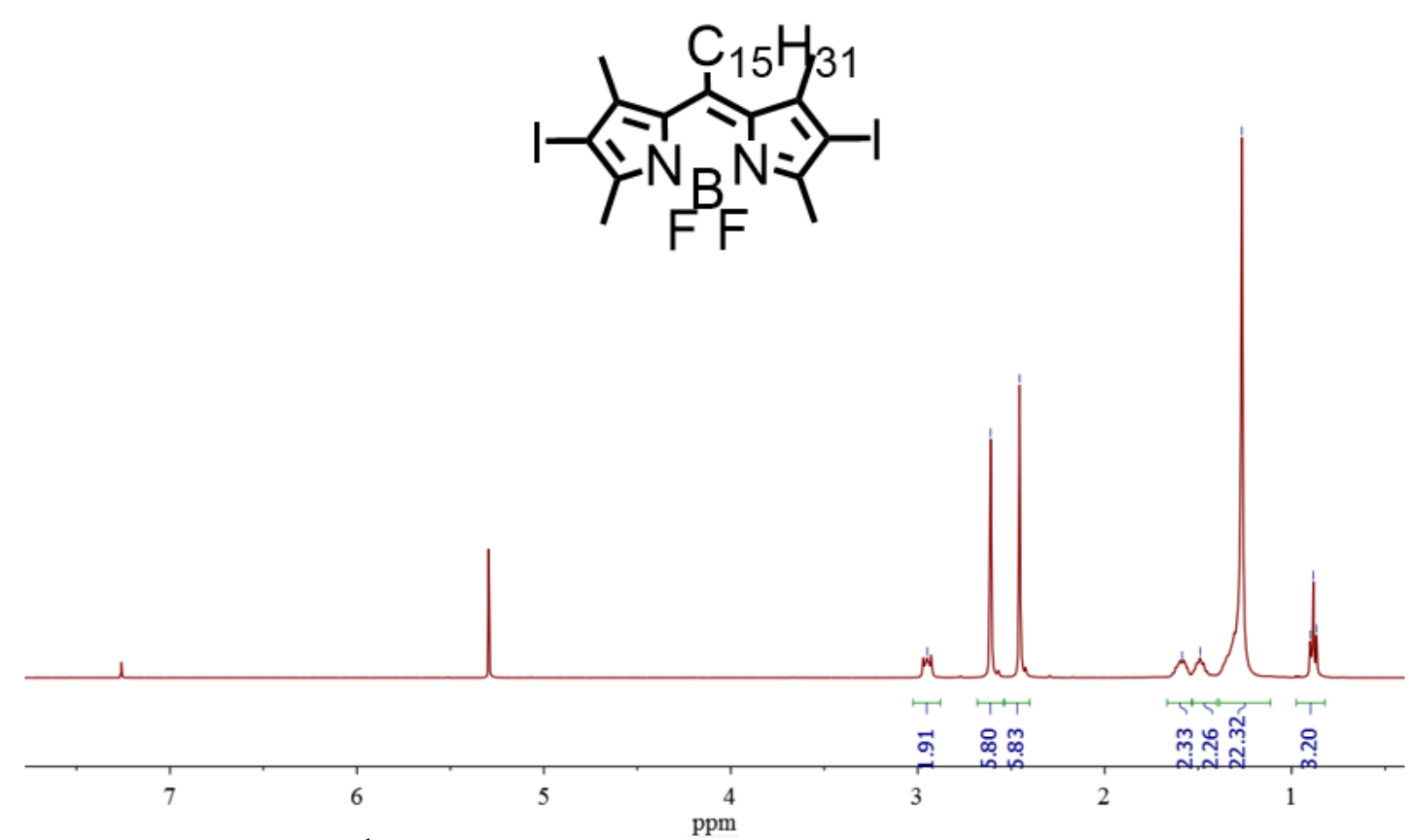

Figure S43. $400 \mathrm{MHz}{ }^{1} \mathrm{H}$ NMR spectrum of boron, difluoro[3-iodo-5-[1-(4-iodo-3,5dimethyl-2H-pyrrol-2-ylidene- $\kappa \mathrm{N}$ )hexadecyl]-2,4-dimethyl-1H-pyrrolato- $\mathrm{N}$ ] in $\mathrm{CDCl}_{3}$
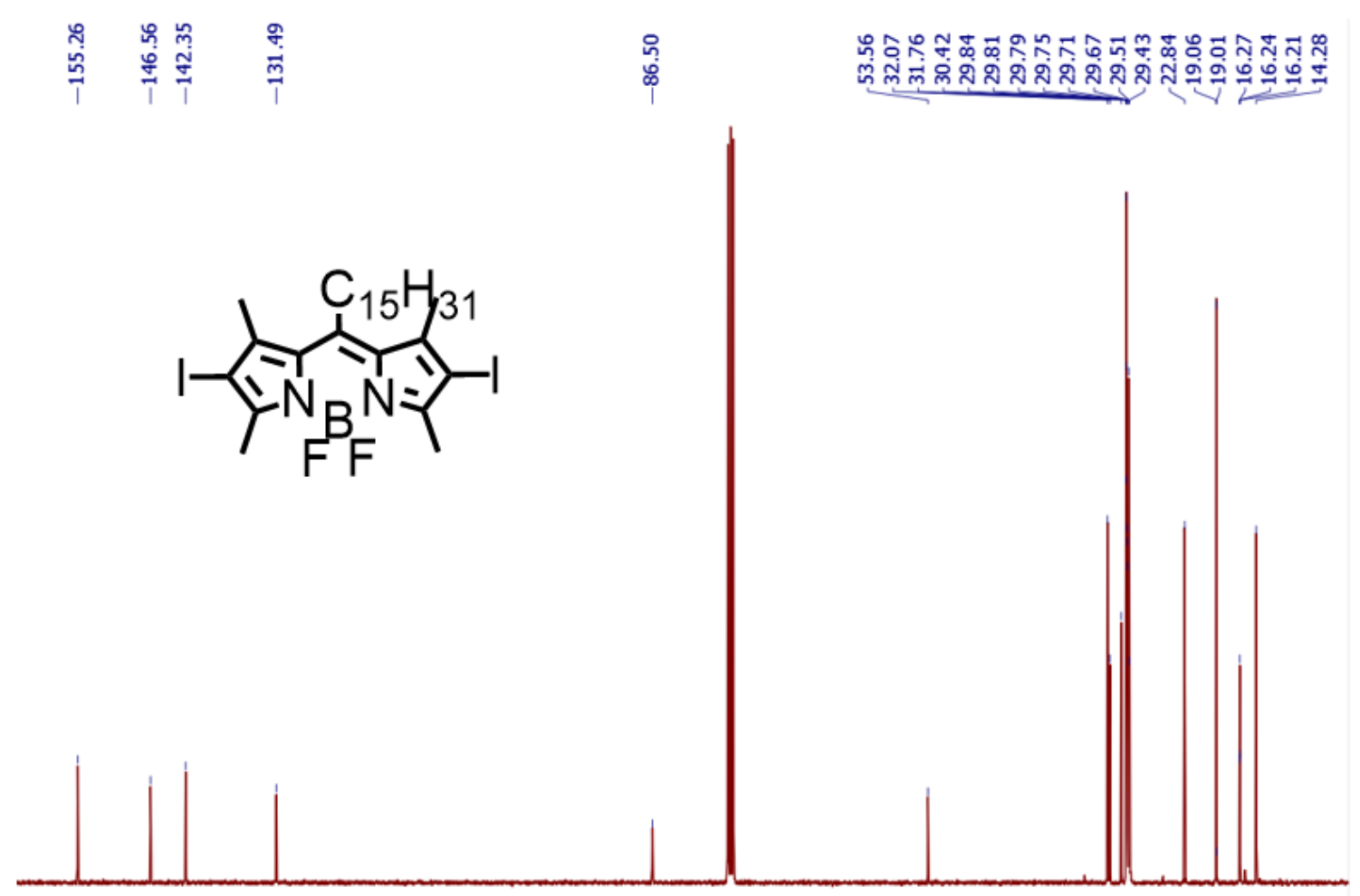

160 140 120 100 ppm $^{80}$ 60 40 20

Figure S44. $400 \mathrm{MHz}{ }^{13} \mathrm{C}$ NMR spectrum of boron, difluoro[3-iodo-5-[1-(4-iodo-3,5dimethyl-2H-pyrrol-2-ylidene- $\mathrm{N}$ )hexadecyl]-2,4-dimethyl-1H-pyrrolato- $\mathrm{N}]$ in $\mathrm{CDCl}_{3}$ 


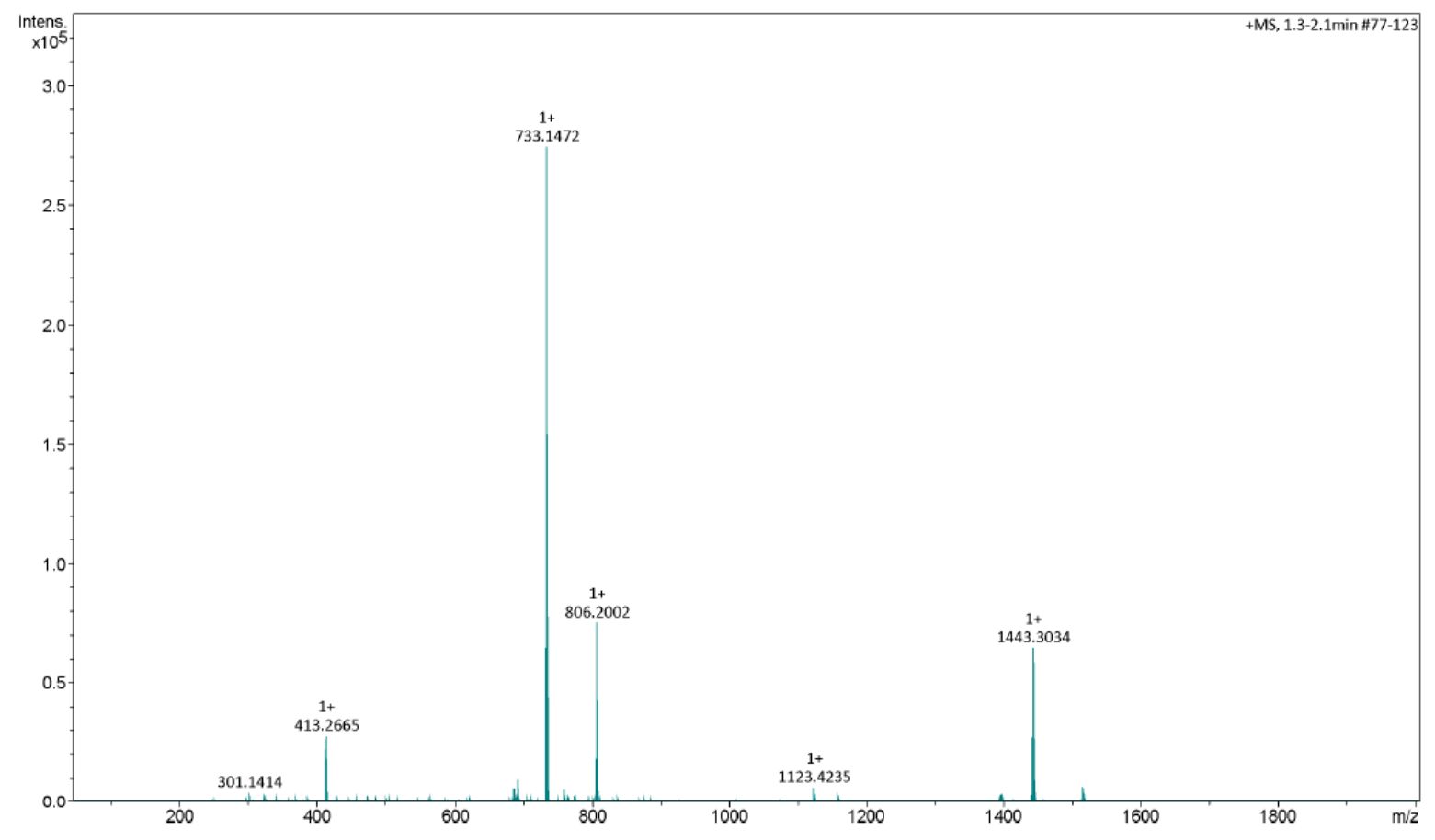

Figure S45. High resolution mass spectrum (ESI+) of boron, difluoro[3-iodo-5-[1-(4iodo-3,5-dimethyl-2H-pyrrol-2-ylidene- $\kappa \mathrm{N}$ )hexadecyl]-2,4-dimethyl-1H-pyrrolato- $\kappa \mathrm{N}$ ]

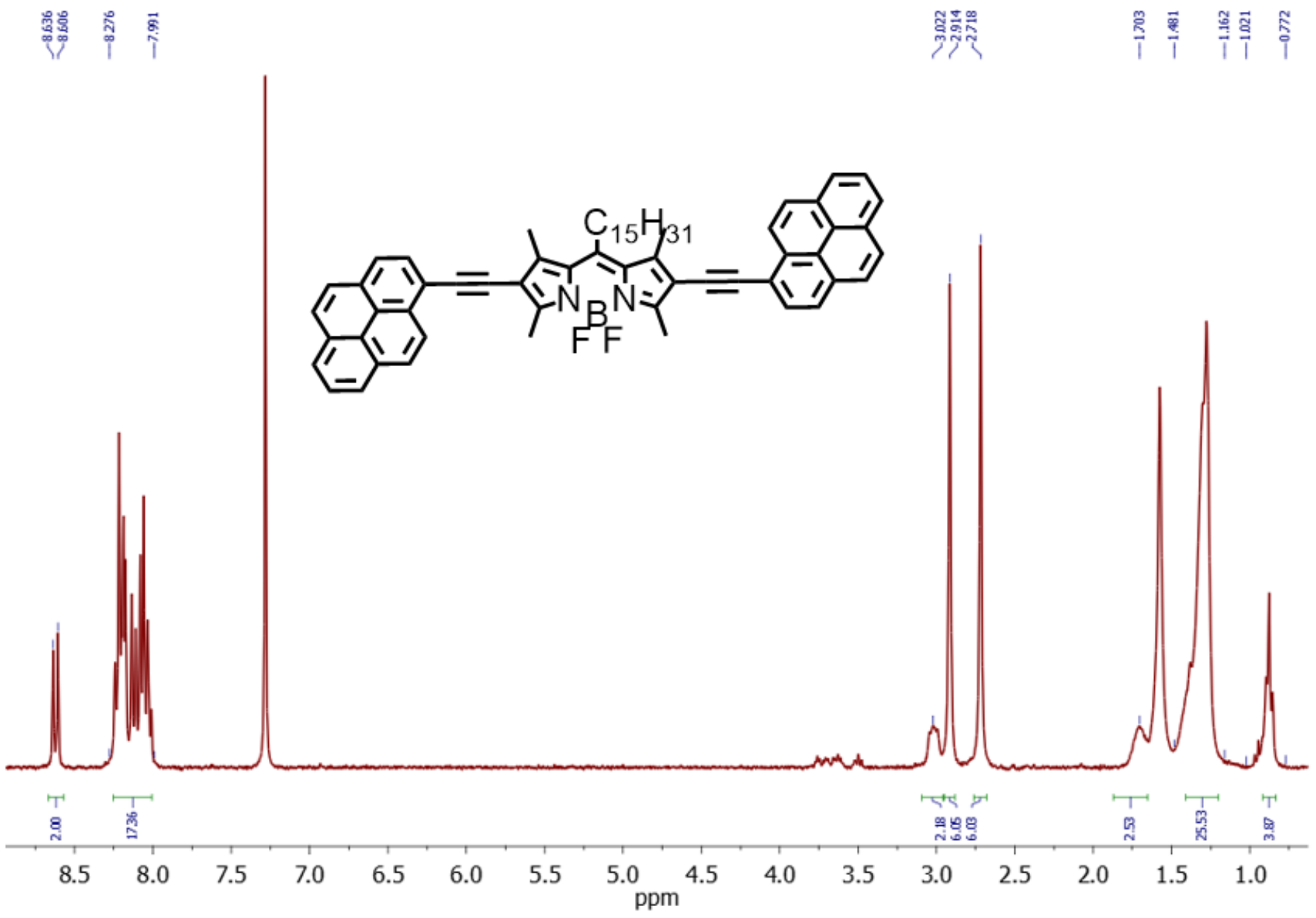

Figure S46. $300 \mathrm{MHz}{ }^{1} \mathrm{H}$ NMR spectrum of $2 \mathrm{PyrBPY}$ in $\mathrm{CDCl}_{3}$ 


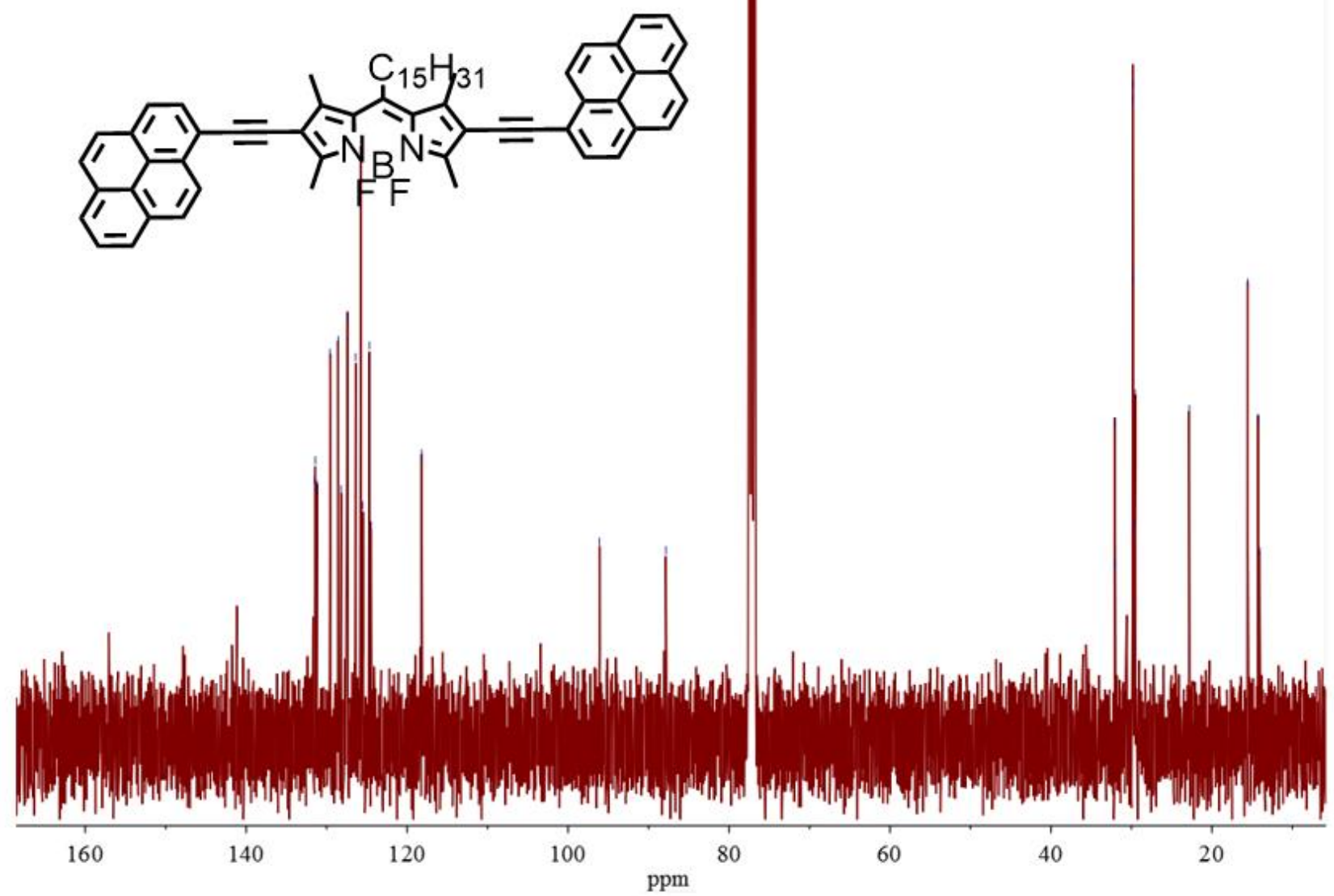

Figure S47. $400 \mathrm{MHz}{ }^{13} \mathrm{C} \mathrm{NMR}$ spectrum of $2 \mathrm{PyrBPY}$ in $\mathrm{CDCl}_{3}$

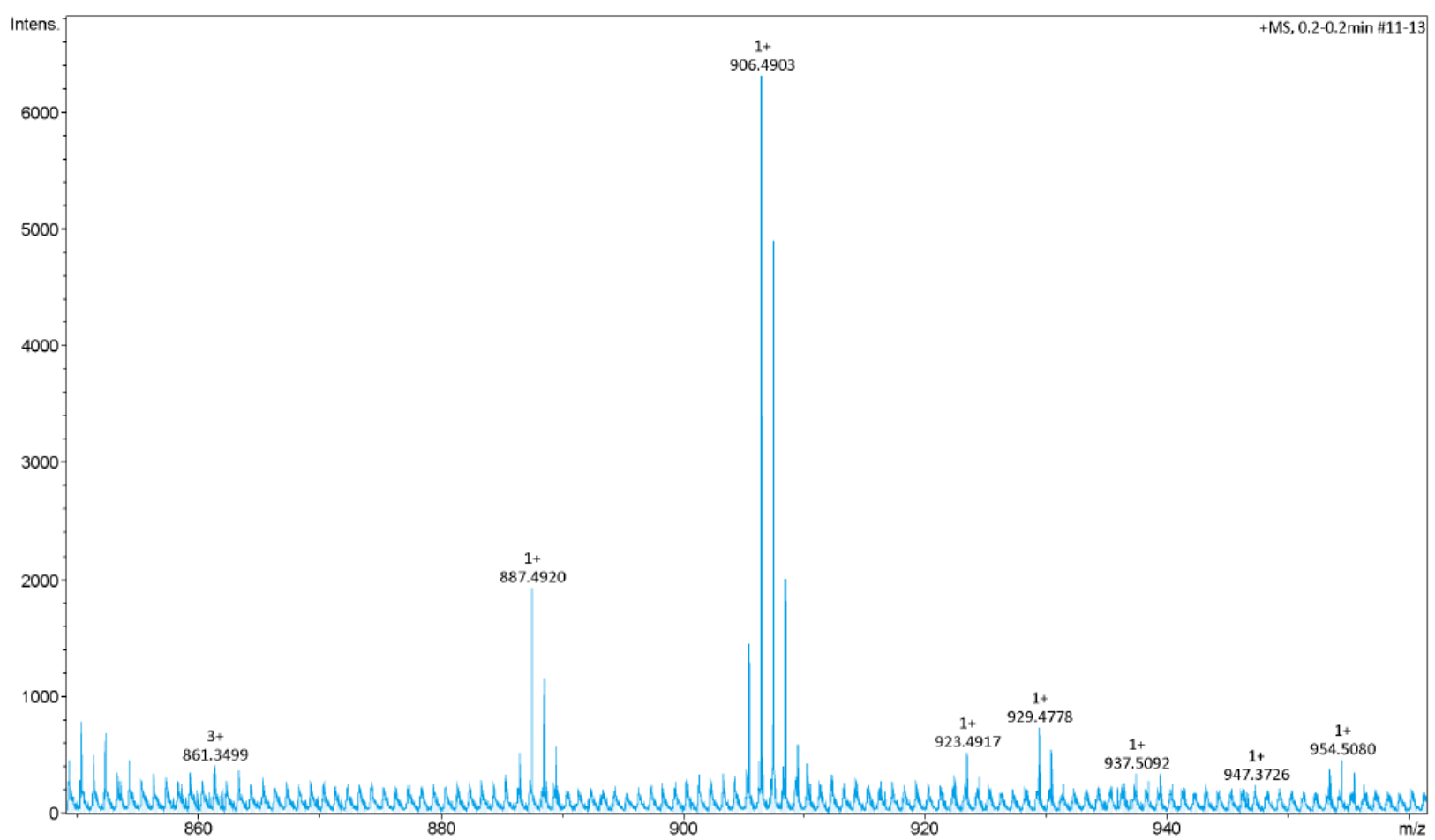

Figure S48. High resolution mass spectrum (ESI+) of 2PyrBPY 


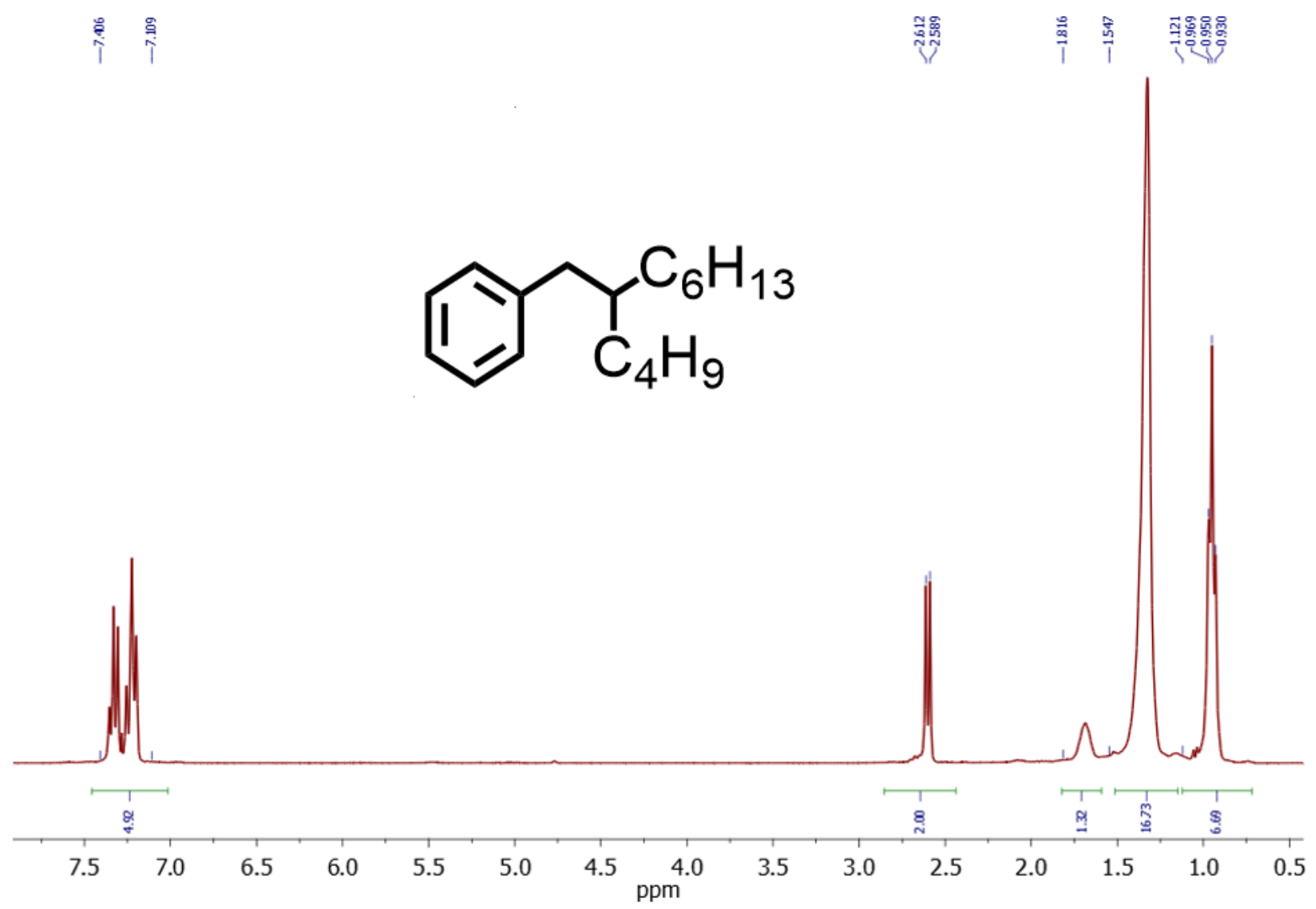

Figure S49. $300 \mathrm{MHz}{ }^{1} \mathrm{H}$ NMR spectrum of 2-butyloctylbenzene in $\mathrm{CDCl}_{3}$

紊

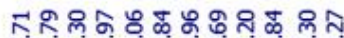

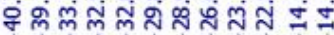
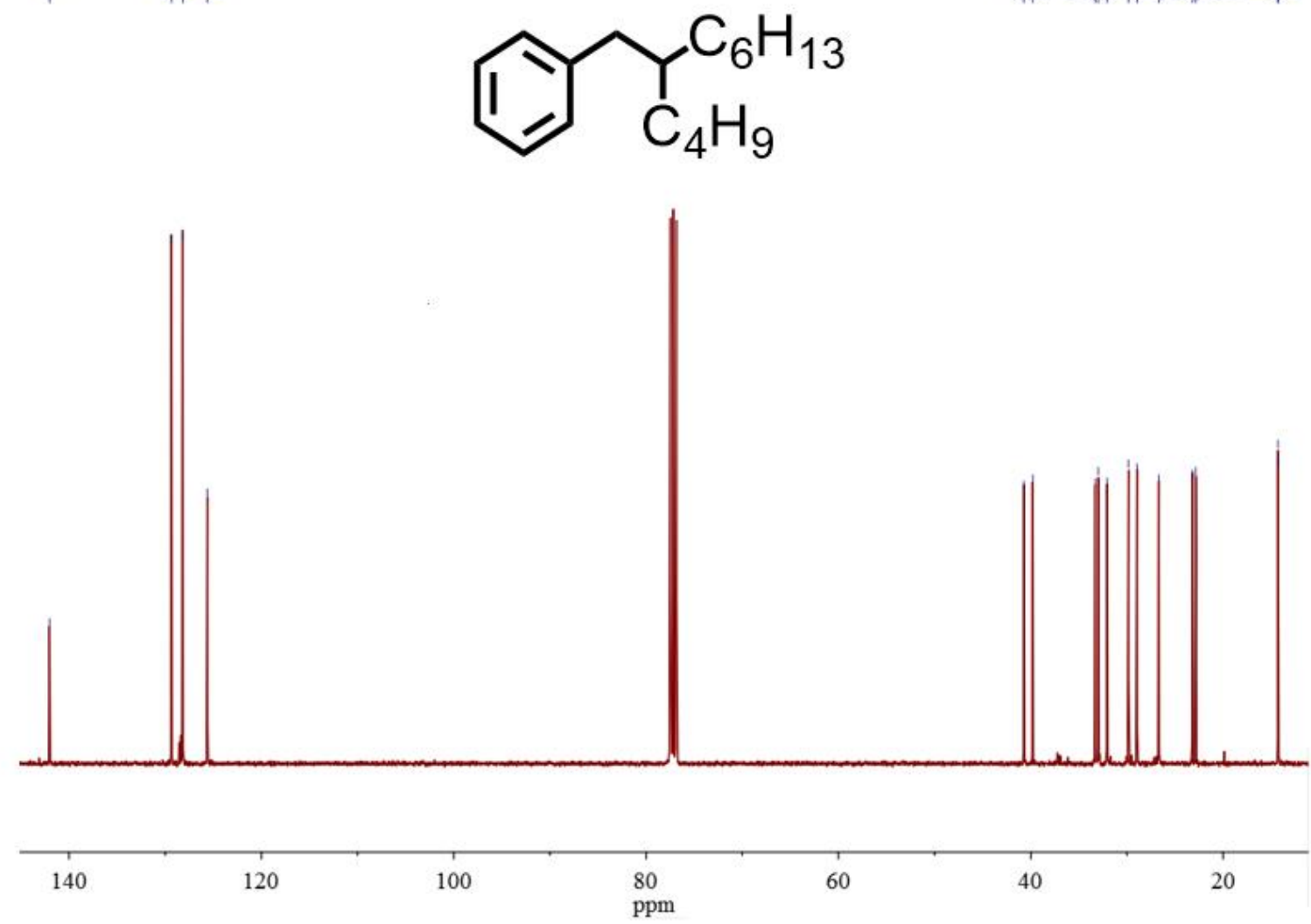

Figure S50. $400 \mathrm{MHz}{ }^{13} \mathrm{C}$ NMR spectrum of 2-butyloctylbenzene in $\mathrm{CDCl}_{3}$

Page S-52 


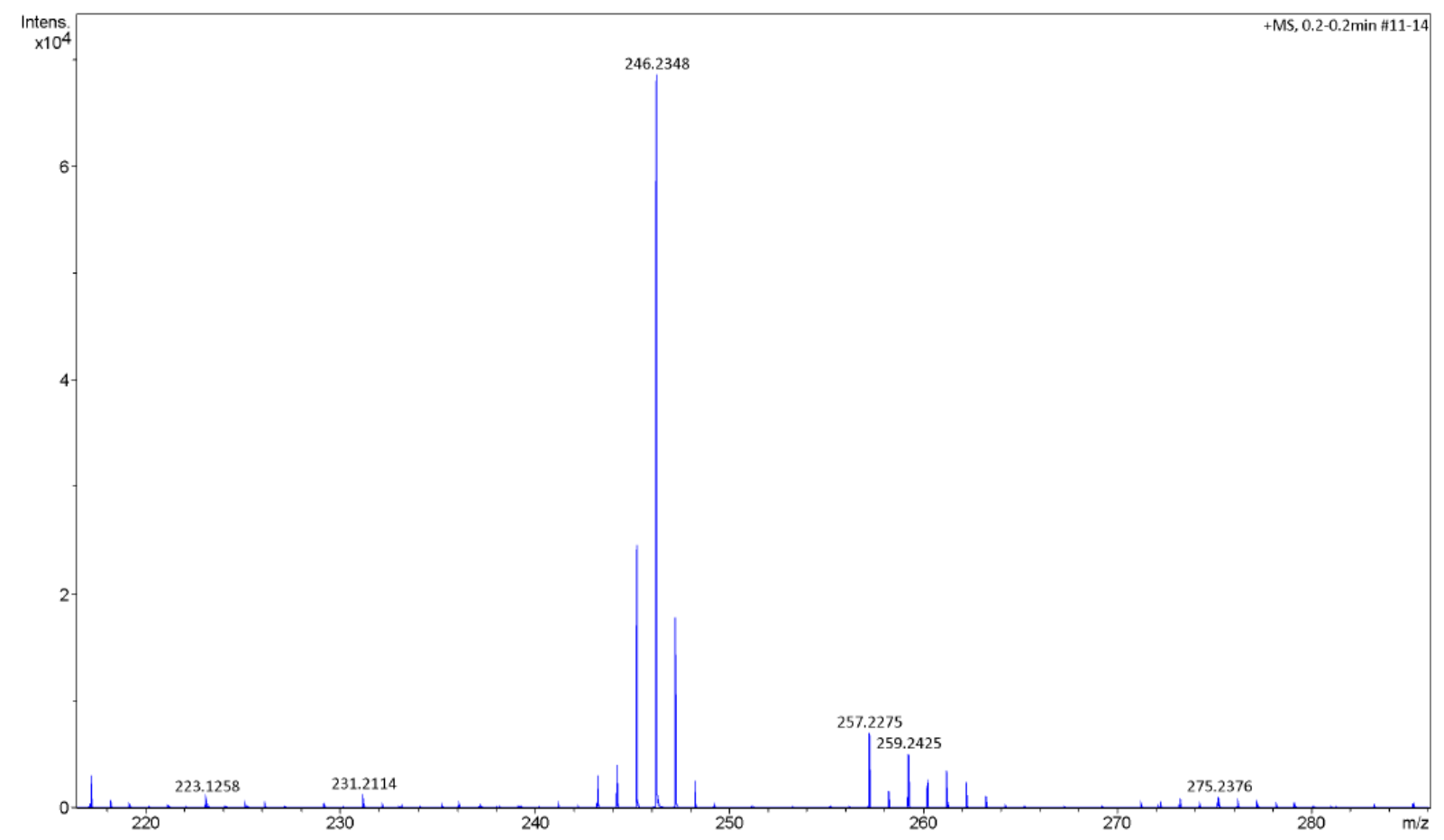

Figure S51. High resolution mass spectrum (APCI+) of 2-butyloctylbenzene

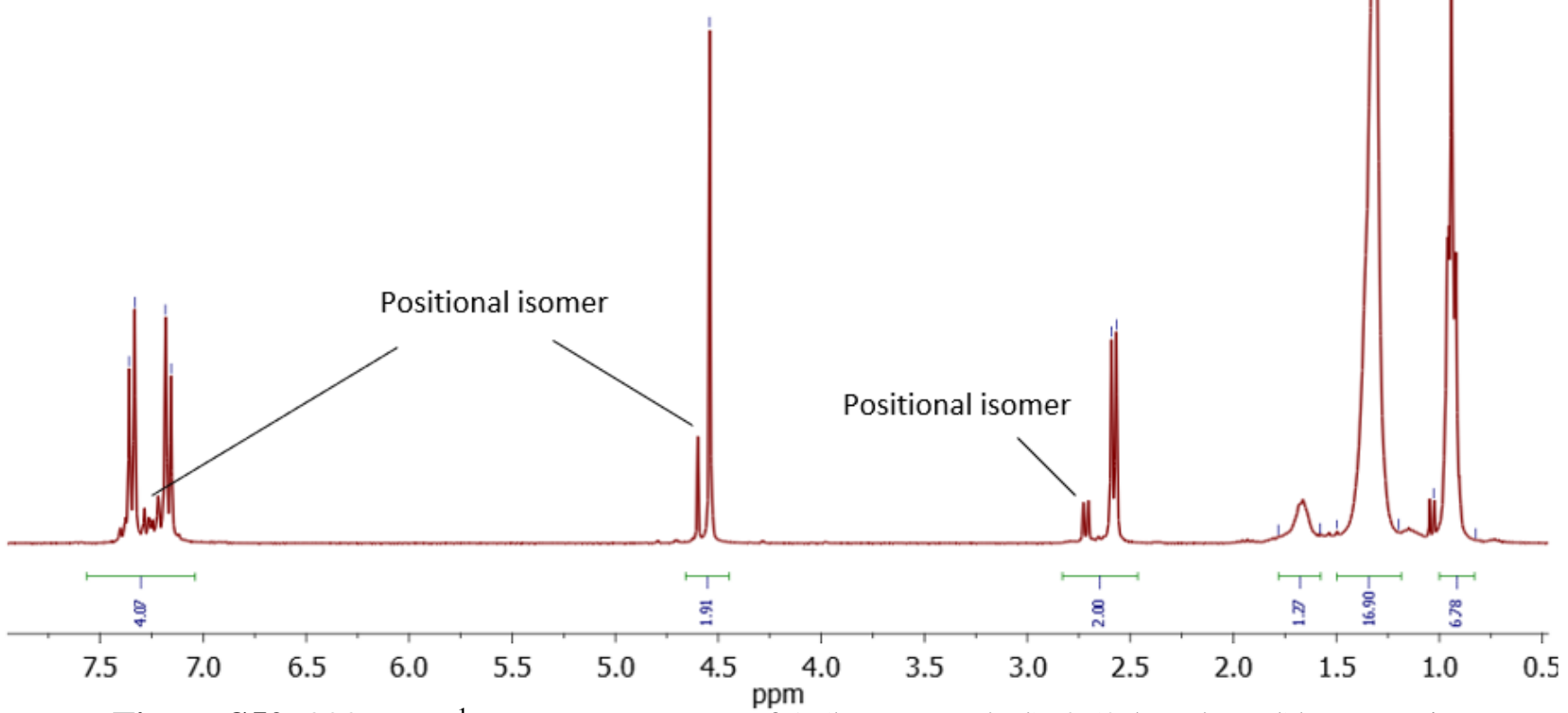

Figure S52. $300 \mathrm{MHz}{ }^{1} \mathrm{H}$ NMR spectrum of 1-(bromomethyl)-4-(2-butyloctyl)benzene in $\mathrm{CDCl}_{3}$ 


\section{Pr) $\mathrm{C}_{4} \mathrm{C}_{6} \mathrm{H}_{13}$}
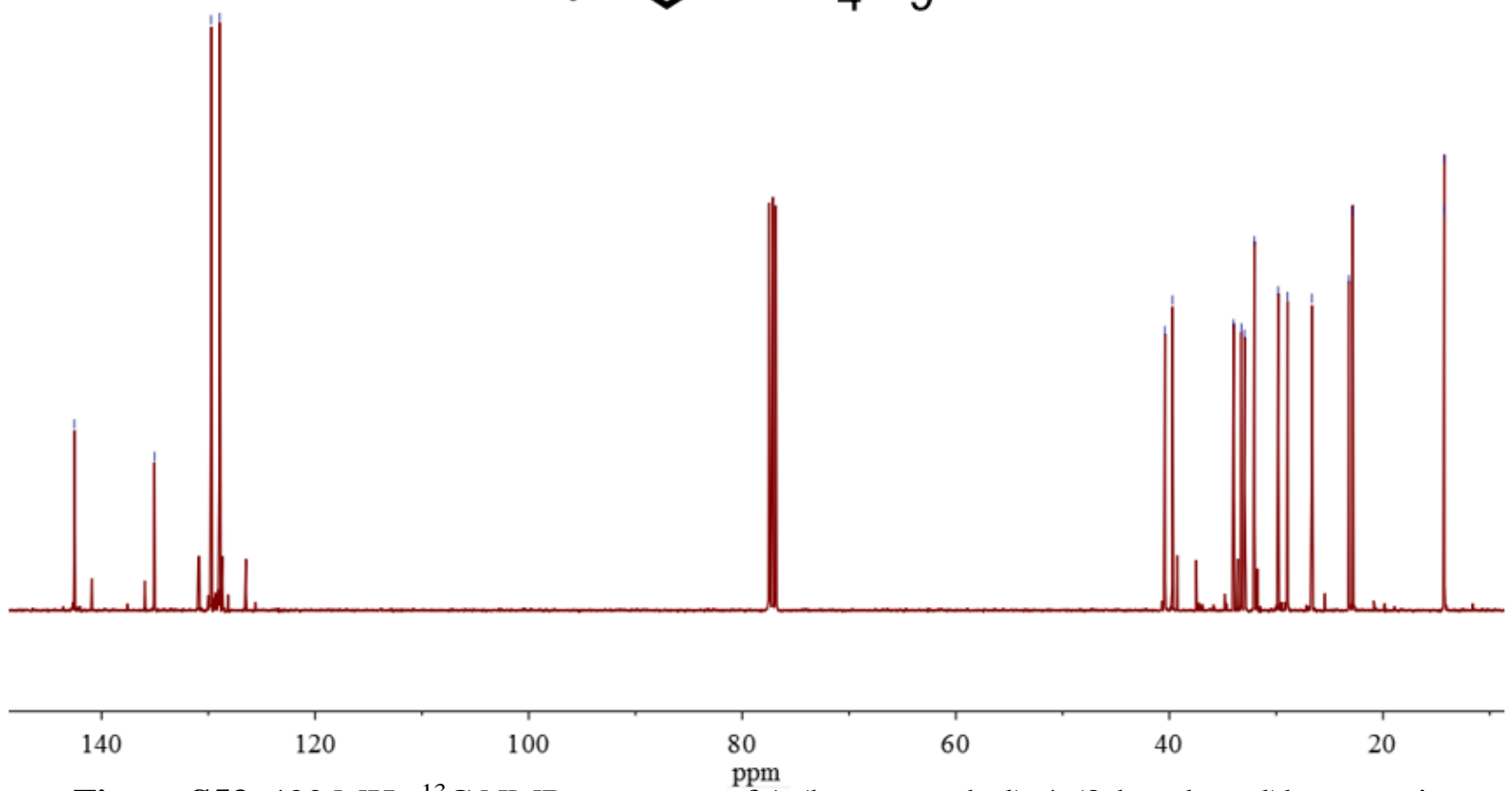

Figure S53. $400 \mathrm{MHz}{ }^{13} \mathrm{C}$ NMR spectrum of 1-(bromomethyl)-4-(2-butyloctyl)benzene in $\mathrm{CDCl}_{3}$

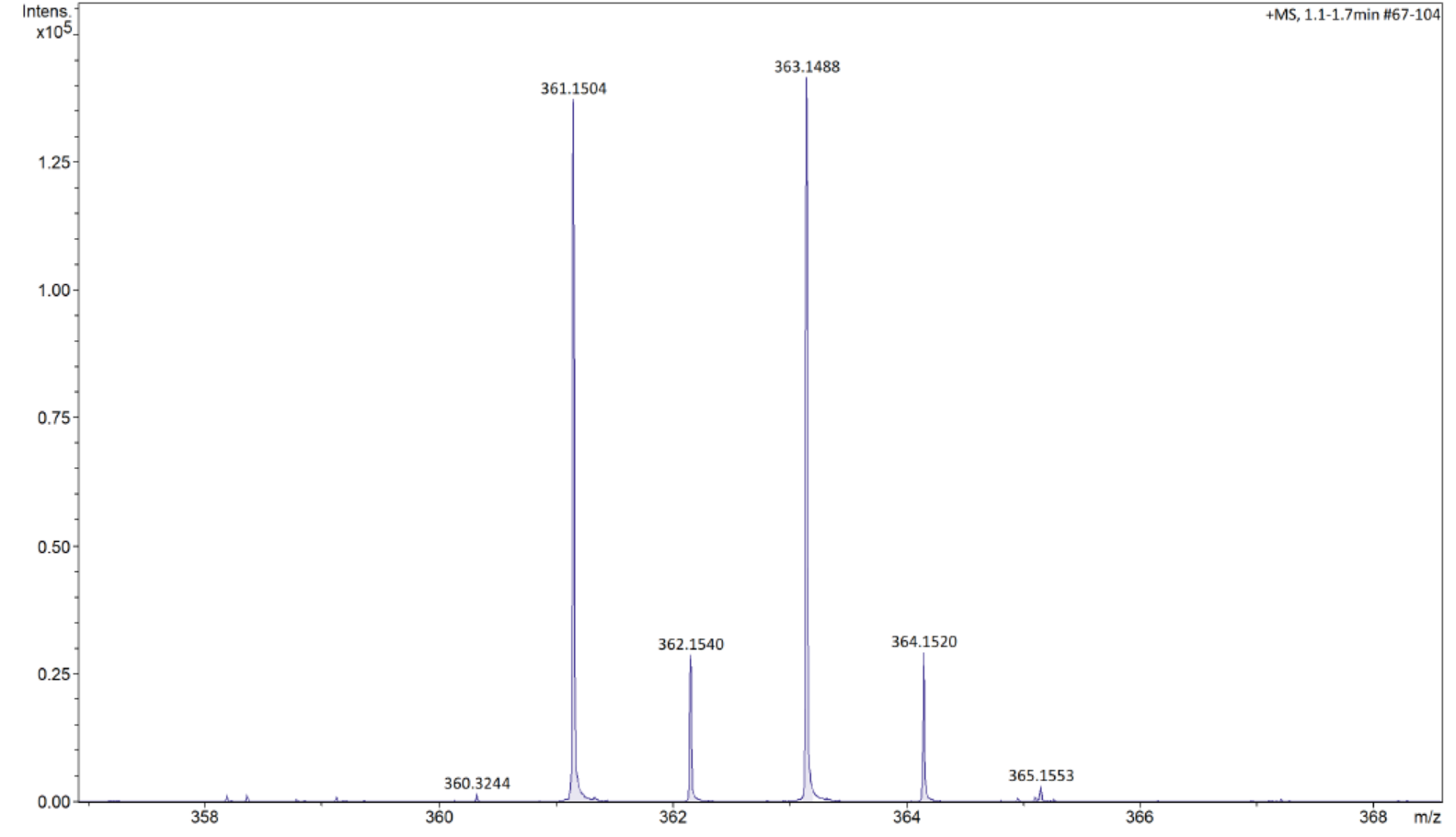

Figure S54. High resolution mass spectrum (ESI+) of 1-(bromomethyl)-4-(2butyloctyl)benzene 


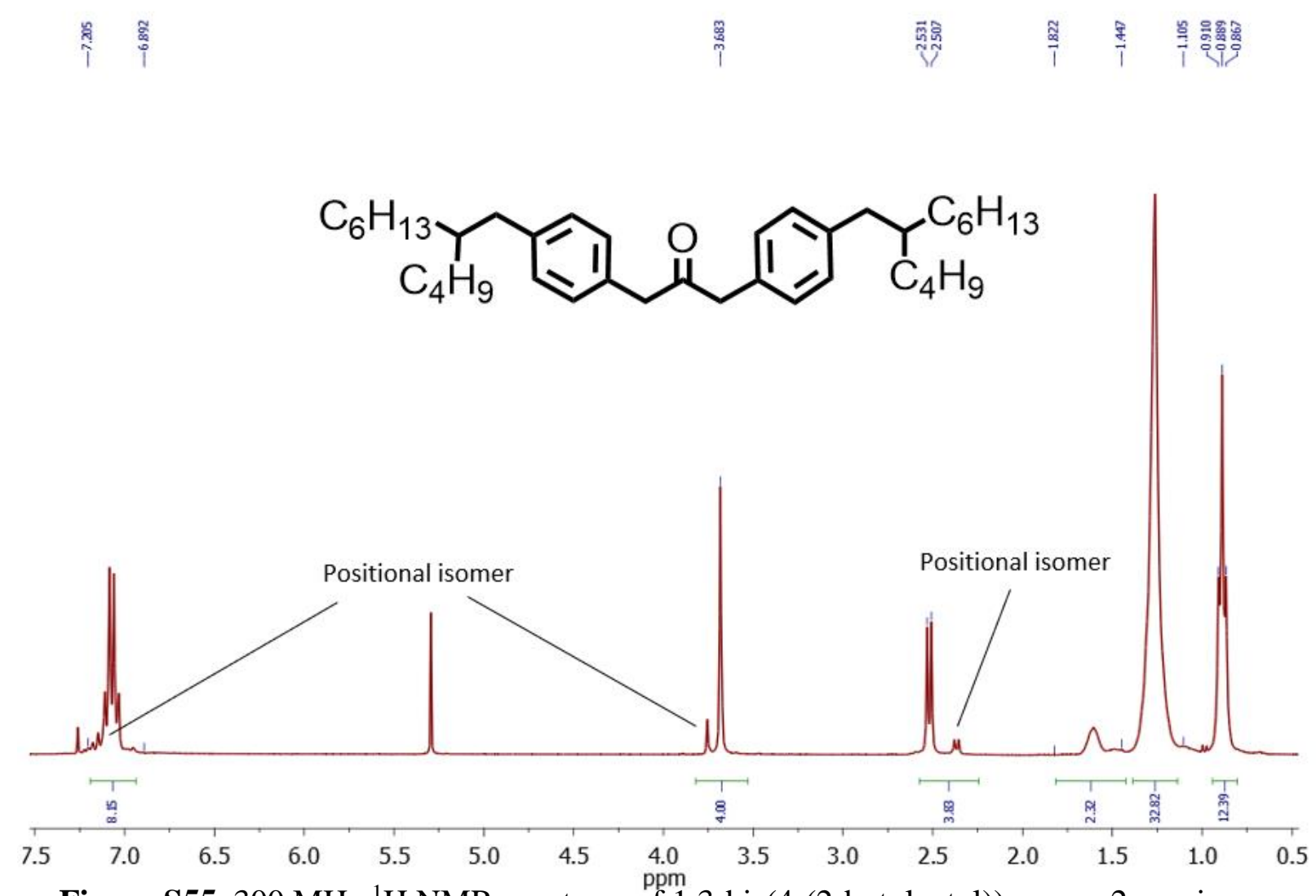

Figure S55. $300 \mathrm{MHz}{ }^{1} \mathrm{H}$ NMR spectrum of 1,3-bis(4-(2-butyloctyl))propan-2-one in $\mathrm{CDCl}_{3}$

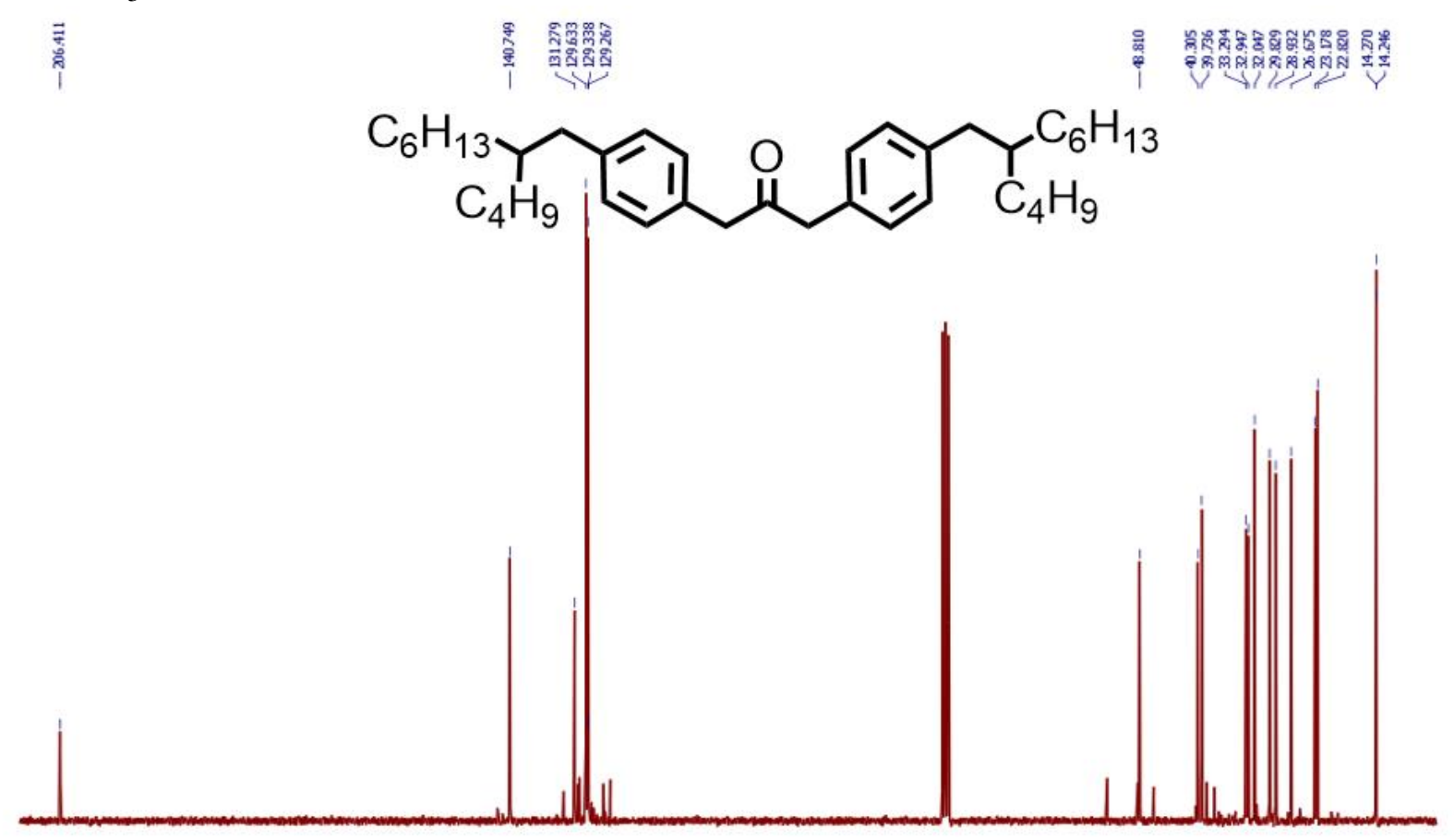

$\begin{array}{lllllllllllllllllllll}210 & 200 & 190 & 180 & 170 & 160 & 150 & 140 & 130 & 120 & \begin{array}{l}110 \\ \mathrm{ppm}\end{array} & 100 & 90 & 80 & 70 & 60 & 50 & 40 & 30 & 20 & 10\end{array}$

Figure S56. $300 \mathrm{MHz}{ }^{13} \mathrm{C}$ NMR spectrum of 1,3-bis(4-(2-butyloctyl))propan-2-one in $\mathrm{CDCl}_{3}$ 


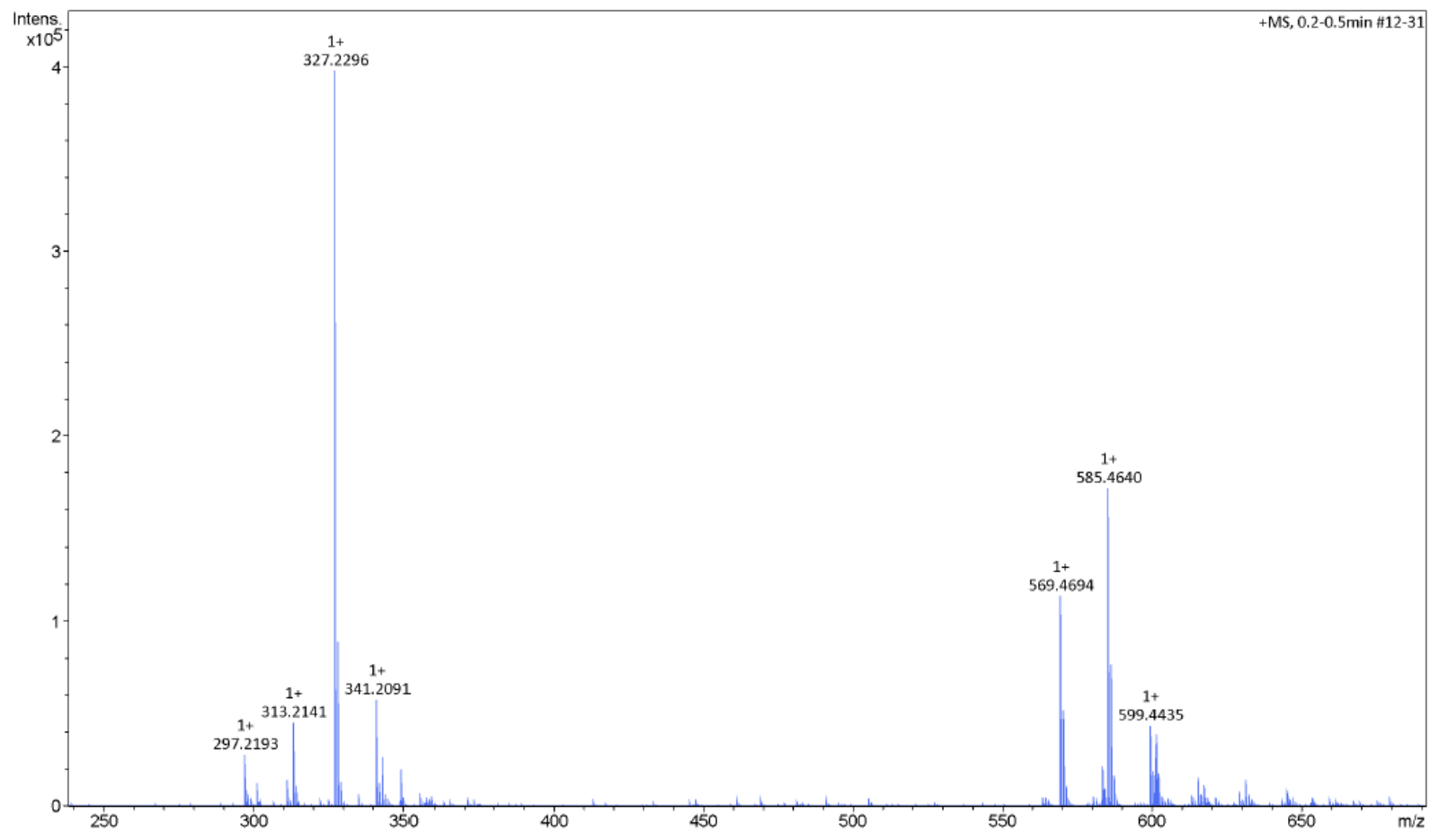

Figure S57. High resolution mass spectrum (ESI+) of 1,3-bis(4-(2-butyloctyl))propan-2-
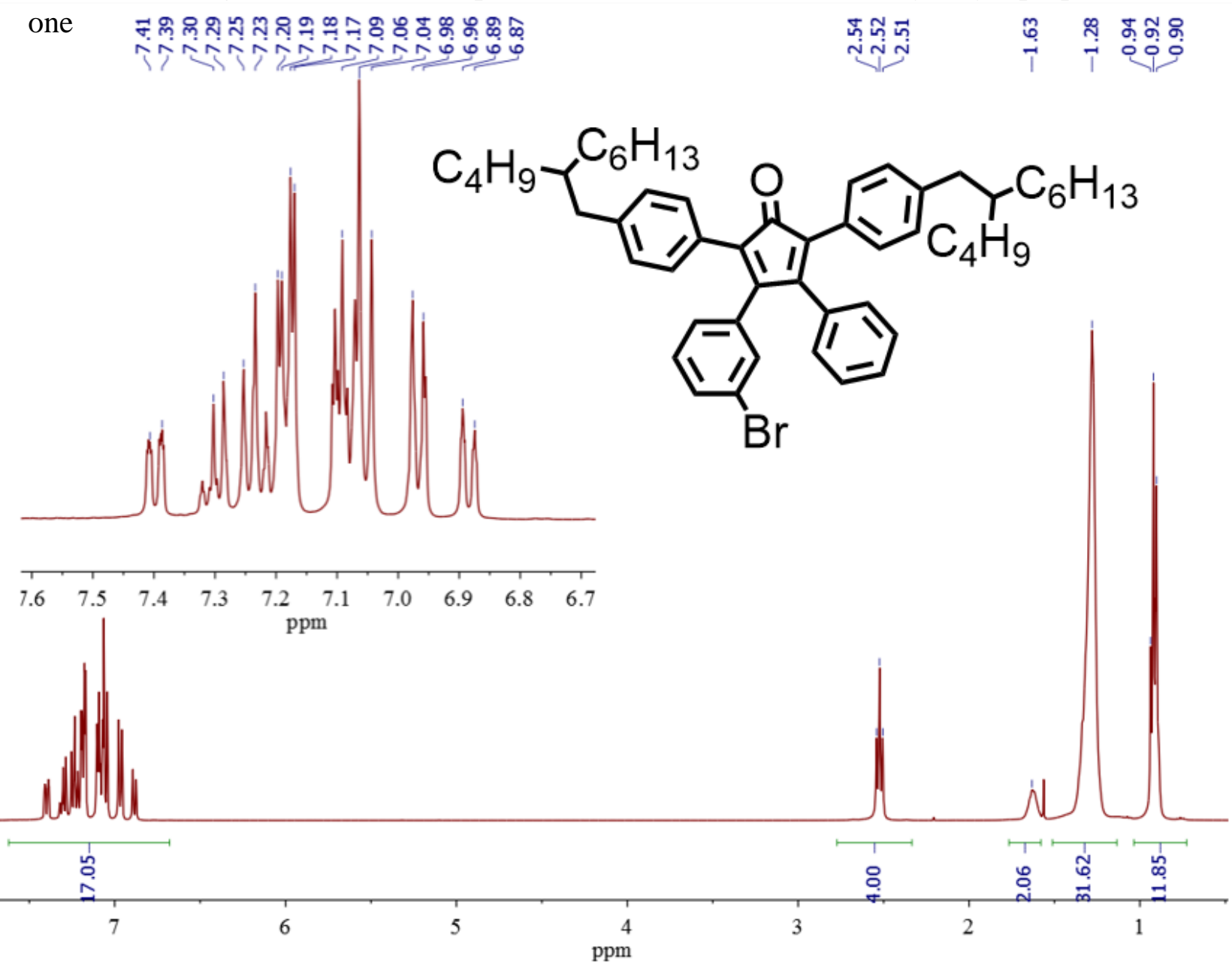

Figure S58. $300 \mathrm{MHz}{ }^{1} \mathrm{H}$ NMR spectrum of 3-(3-bromophenyl)-2,5-bis(4-(2-

butyloctyl)phenyl)-4-phenylcyclopenta-2,4-dienone in $\mathrm{CDCl}_{3}$ 

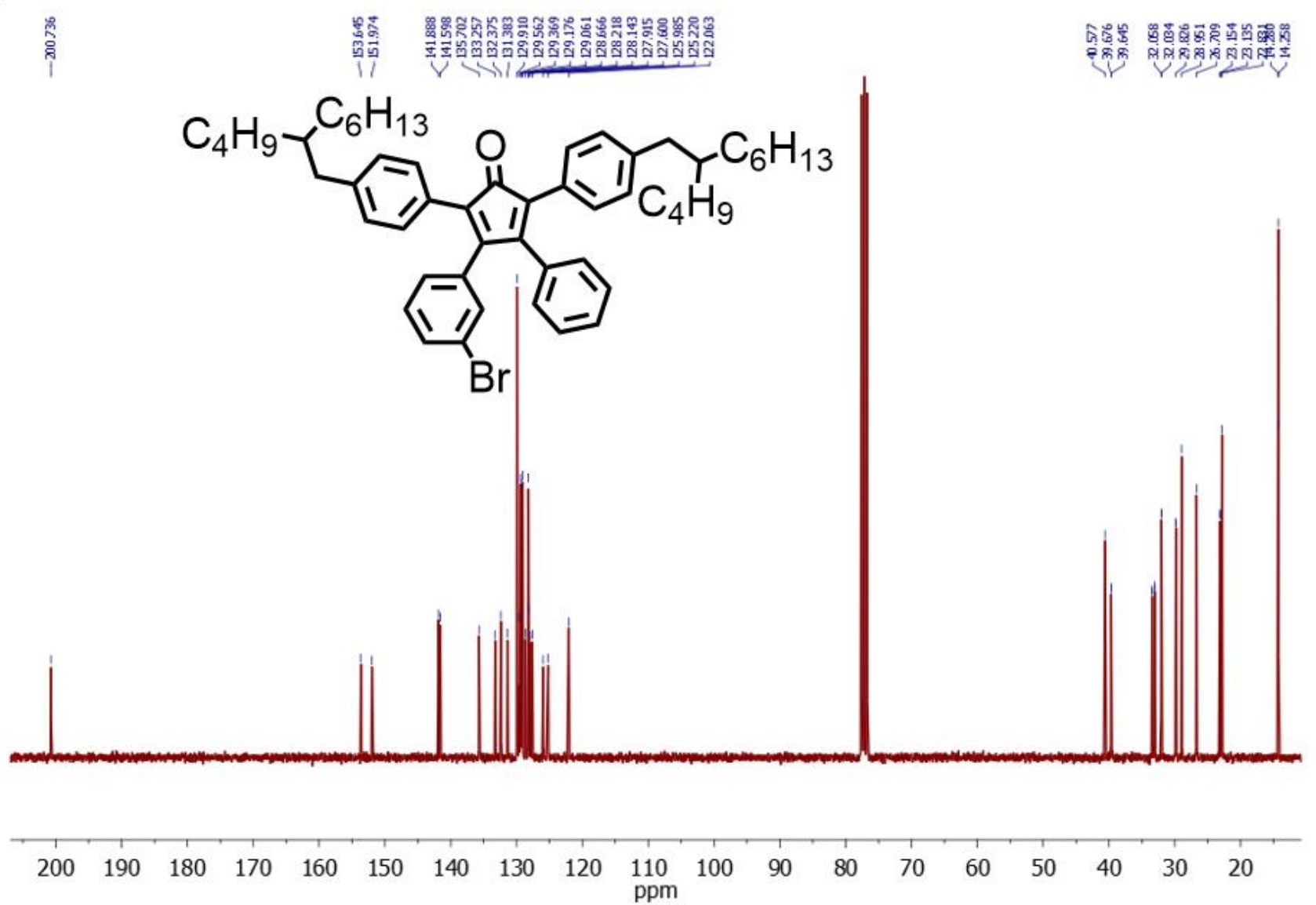

Figure S59. $300 \mathrm{MHz}{ }^{13} \mathrm{C}$ NMR spectrum of 3-(3-bromophenyl)-2,5-bis(4-(2-

butyloctyl)phenyl)-4-phenylcyclopenta-2,4-dienone in $\mathrm{CDCl}_{3}$

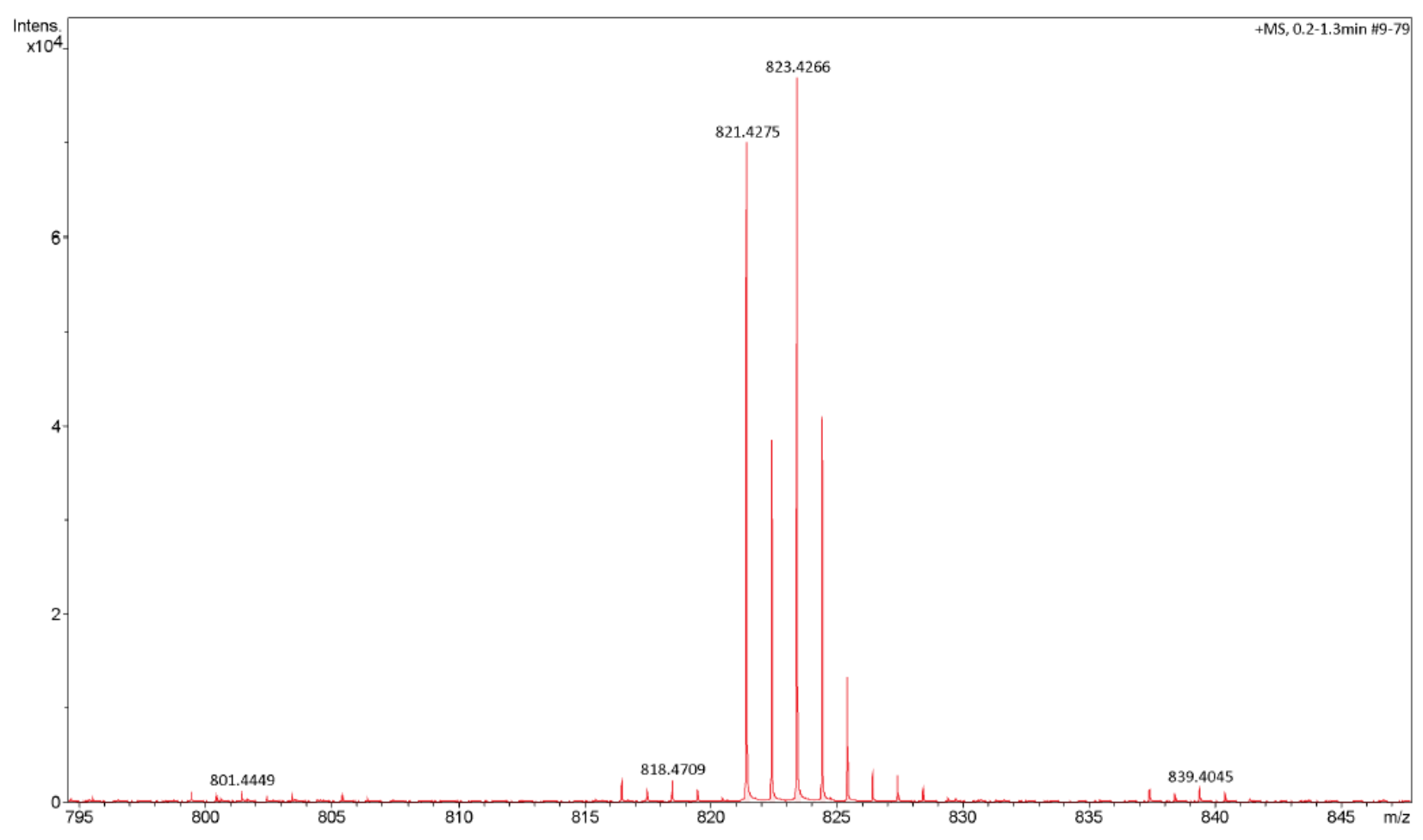

Figure S60. High resolution mass spectrum (ESI+) of 3-(3-bromophenyl)-2,5-bis(4-(2butyloctyl)phenyl)-4-phenylcyclopenta-2,4-dienone 


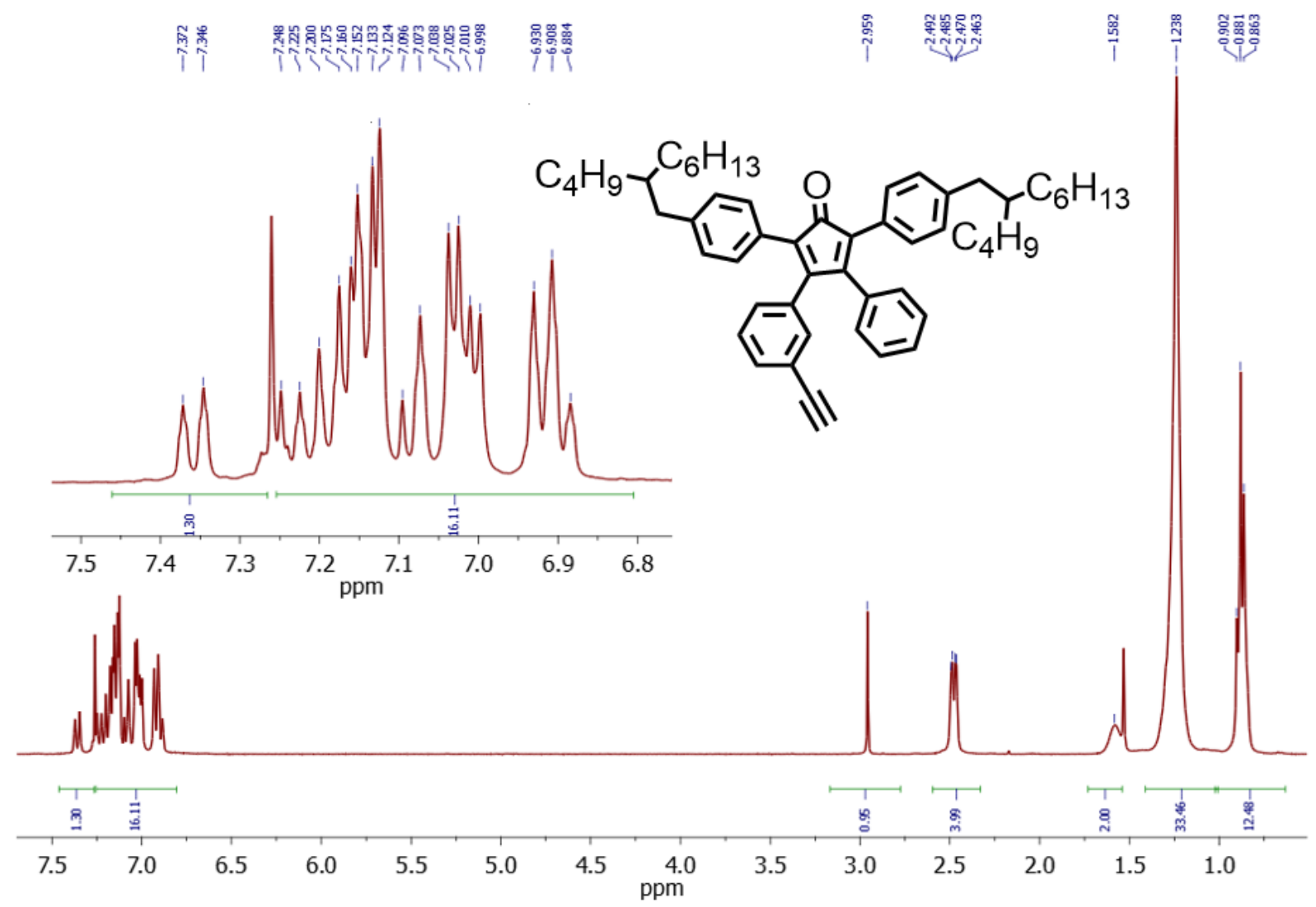

Figure S61. $300 \mathrm{MHz}{ }^{1} \mathrm{H}$ NMR spectrum of 3-(3-ethynylphenyl)-2,5-bis(4-(2butyloctyl)phenyl)-4-phenylcyclopenta-2,4-dienone in $\mathrm{CDCl}_{3}$

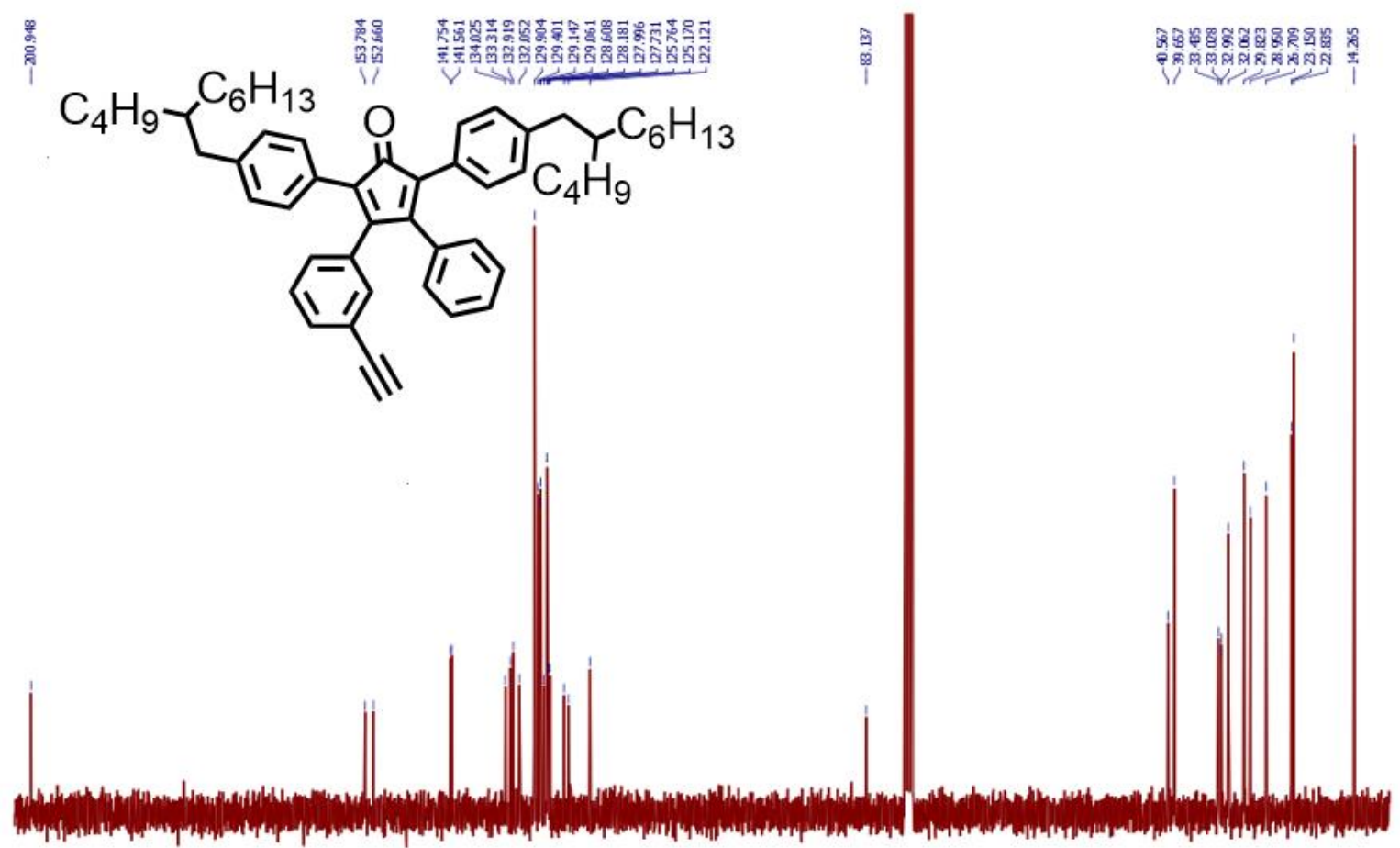

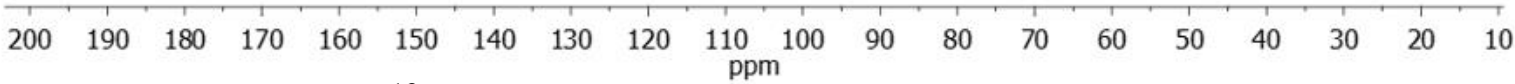

Figure S62. $300 \mathrm{MHz}{ }^{13} \mathrm{C}$ NMR spectrum of 3-(3-ethynylphenyl)-2,5-bis(4-(2butyloctyl)phenyl)-4-phenylcyclopenta-2,4-dienone in $\mathrm{CDCl}_{3}$ 


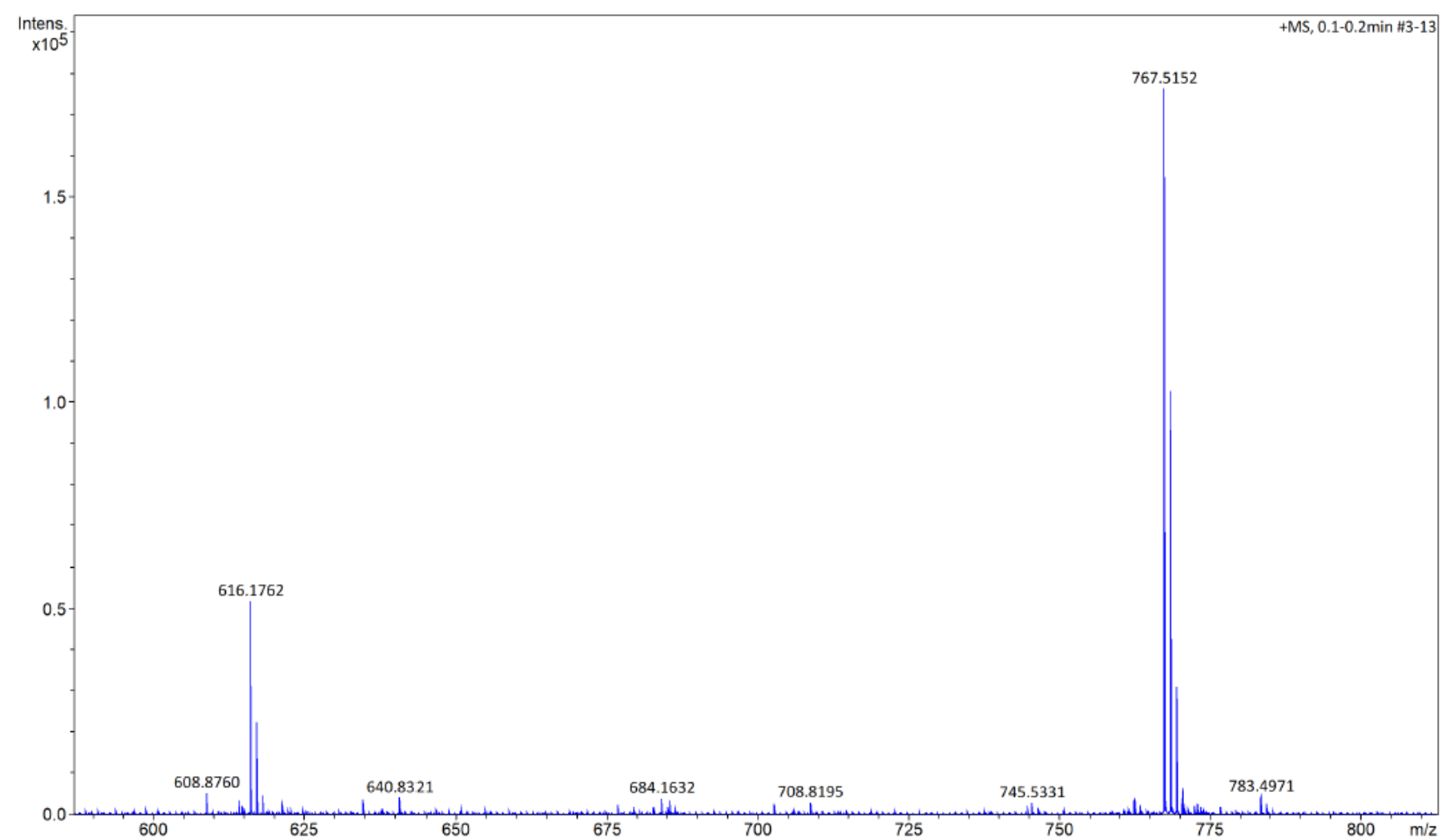

Figure S63. High resolution mass spectrum (ESI+) of 3-(3-ethynylphenyl)-2,5-bis(4-(2butyloctyl)phenyl)-4-phenylcyclopenta-2,4-dienone
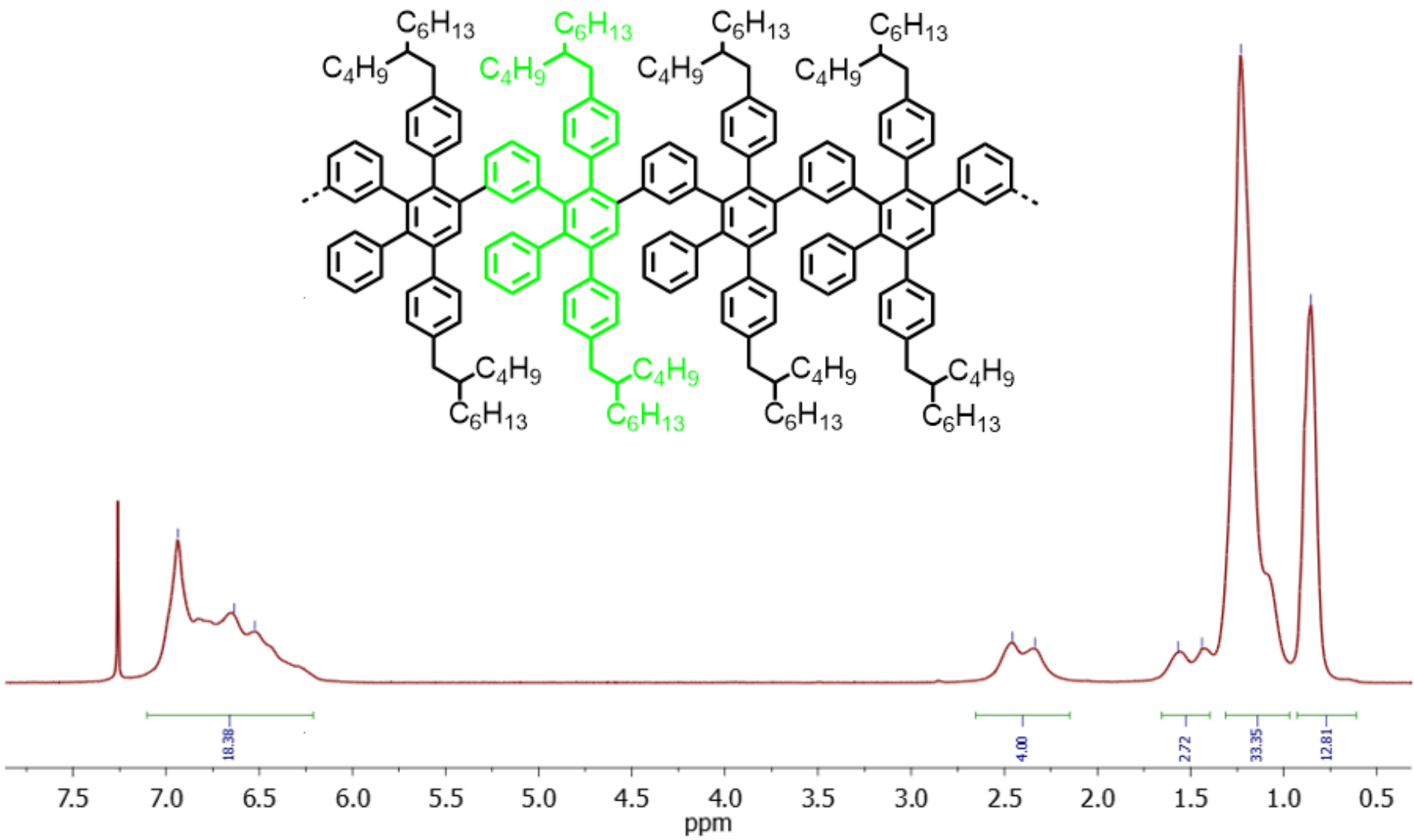

Figure S64. $300 \mathrm{MHz}{ }^{1} \mathrm{H} \mathrm{NMR}$ spectrum of GNR precursor in $\mathrm{CDCl}_{3}$ 


\section{References}

(1) Li, D.; Müller, M. B.; Gilje, S.; Kaner, R. B.; Wallace, G. G. Processable Aqueous Dispersions of Graphene Nanosheets. Nat. Nanotechnol. 2008, 3 (2), 101-105. https://doi.org/10.1038/nnano.2007.451.

(2) Gao, K.; Miao, J.; Xiao, L.; Deng, W.; Kan, Y.; Liang, T.; Wang, C.; Huang, F.; Peng, J.; Cao, Y.; et al. Multi-Length-Scale Morphologies Driven by Mixed Additives in Porphyrin-Based Organic Photovoltaics. Adv. Mater. 2016, 28 (23), 4727-4733. https://doi.org/10.1002/adma.201505645.

(3) Stark, T.; Suhartono, M.; Göbel, M. W.; Lautens, M. A Palladium-Catalyzed Domino Reaction as Key Step for the Synthesis of Functionalized Aromatic Amino Acids. Synlett 2013, 24 (20), 2730-2734. https://doi.org/10.1055/s-00331339892.

(4) Wang, Y.; Li, X.; Li, F.; Sun, W. Y.; Zhu, C.; Cheng, Y. Strong Circularly Polarized Luminescence Induced from Chiral Supramolecular Assembly of Helical Nanorods. Chem. Commun. 2017, 53 (54), 7505-7508.

https://doi.org/10.1039/c7cc04363e.

(5) Lee, O. P.; Yiu, A. T.; Beaujuge, P. M.; Woo, C. H.; Holcombe, T. W.; Millstone, J. E.; Douglas, J. D.; Chen, M. S.; Fréchet, J. M. J. Efficient Small Molecule Bulk Heterojunction Solar Cells with High Fill Factors via Pyrene-Directed Molecular Self-Assembly. Adv. Mater. 2011, 23 (45), 5359-5363.

https://doi.org/10.1002/adma.201103177.

(6) Aldrich, T. J.; Dudnik, A. S.; Eastham, N. D.; Manley, E. F.; Chen, L. X.; Chang, R. P. H.; Melkonyan, F. S.; Facchetti, A.; Marks, T. J. Suppressing Defect Formation Pathways in the Direct C-H Arylation Polymerization of Photovoltaic Copolymers. Macromolecules 2018, 51 (22), 9140-9155. https://doi.org/10.1021/acs.macromol.8b02297.

(7) Jiménez, Á. J.; Sekita, M.; Caballero, E.; Marcos, M. L.; Rodríguez-Morgade, M. S.; Guldi, D. M.; Torres, T. Assembling a Phthalocyanine and Perylenediimide Donor-Acceptor Hybrid through a Platinum(II) Diacetylide Linker. Chem. - A Eur. J. 2013, 19 (43), 14506-14514. https://doi.org/10.1002/chem.201301630.

(8) Kilic, B.; Yesilgul, N.; Polat, V.; Gercek, Z.; Akkaya, E. U. Bodipy-Based Photosensitizers with Long Alkyl Tails at the Meso Position: Efficient Singlet Oxygen Generation in Cremophor-EL Micelles. Tetrahedron Lett. 2016, 57 (12), 1317-1320. https://doi.org/10.1016/j.tetlet.2016.02.033.

(9) Souza, B. S.; Leopoldino, E. C.; Tondo, D. W.; Dupont, J.; Nome, F. ImidazoliumBased Zwitterionic Surfactant: A New Amphiphilic Pd Nanoparticle Stabilizing Agent. Langmuir 2012, 28 (1), 833-840. https://doi.org/10.1021/la203501f.

(10) Yan, X.; Cui, X.; Li, B.; Li, L. S. Large, Solution-Processable Graphene Quantum Dots as Light Absorbers for Photovoltaics. Nano Lett. 2010, 10 (5), 1869-1873. 
https://doi.org/10.1021/nl101060h.

(11) Narita, A.; Feng, X.; Hernandez, Y.; Jensen, S. A.; Bonn, M.; Yang, H.; Verzhbitskiy, I. A.; Casiraghi, C.; Hansen, M. R.; Koch, A. H. R.; et al. Synthesis of Structurally Well-Defined and Liquid-Phase-Processable Graphene Nanoribbons. Nat. Chem. 2014, 6 (2), 126-132. https://doi.org/10.1038/nchem.1819.

(12) Snellenburg, J. J.; Laptenok, S.; Seger, R.; Mullen, K. M.; van Stokkum, I. H. M. Glotaran: A Java-Based Graphical User Interface for the R Package TIMP. J. Stat. Softw. 2012, 49 (1), 1-22. https://doi.org/10.18637/jss.v049.i03.

(13) Giannozzi, P.; Baroni, S.; Bonini, N.; Calandra, M.; Car, R.; Cavazzoni, C.; Ceresoli, D.; Chiarotti, G. L.; Cococcioni, M.; Dabo, I.; et al. QUANTUM ESPRESSO: A Modular and Open-Source Software Project for Quantum Simulations of Materials. J. Phys. Condens. Matter 2009, 21 (39), 395502. https://doi.org/10.1088/0953-8984/21/39/395502.

(14) Giannozzi, P.; Andreussi, O.; Brumme, T.; Bunau, O.; Buongiorno Nardelli, M.; Calandra, M.; Car, R.; Cavazzoni, C.; Ceresoli, D.; Cococcioni, M.; et al. Advanced Capabilities for Materials Modelling with Quantum ESPRESSO. $J$. Phys. Condens. Matter 2017, 29 (46), 465901. https://doi.org/10.1088/1361648X/aa8f79.

(15) Perdew, J. P.; Burke, K.; Ernzerhof, M. Generalized Gradient Approximation Made Simple. Phys. Rev. Lett. 1996, 77 (18), 3865-3868. https://doi.org/10.1103/PhysRevLett.77.3865.

(16) Dal Corso, A. Pseudopotentials Periodic Table: From H to Pu. Comput. Mater. Sci. 2014, 95, 337-350. https://doi.org/10.1016/j.commatsci.2014.07.043.

(17) Malcioğlu, O. B.; Gebauer, R.; Rocca, D.; Baroni, S. TurboTDDFT - A Code for the Simulation of Molecular Spectra Using the Liouville-Lanczos Approach to Time-Dependent Density-Functional Perturbation Theory. Comput. Phys. Commun. 2011, 182 (8), 1744-1754. https://doi.org/10.1016/j.cpc.2011.04.020.

(18) Ge, X.; Binnie, S. J.; Rocca, D.; Gebauer, R.; Baroni, S. TurboTDDFT 2.0 Hybrid Functionals and New Algorithms within Time-Dependent DensityFunctional Perturbation Theory. Comput. Phys. Commun. 2014, 185 (7), 20802089. https://doi.org/10.1016/j.cpc.2014.03.005.

(19) Tanguy, L.; Hetru, O.; Langlois, A.; Harvey, P. D. Characterization and Minimization of Glaser Competitive Homocoupling in Sonogashira PorphyrinBased Polycondensation. J. Org. Chem. 2019, 84 (6), 3590-3594. https://doi.org/10.1021/acs.joc.8b02596.

(20) Wang, L.; Wang, J. W.; Cui, A. J.; Cai, X. X.; Wan, Y.; Chen, Q.; He, M. Y.; Zhang, W. Regioselective 2,6-Dihalogenation of BODIPYs in 1,1,1,3,3,3Hexafluoro-2- Propanol and Preparation of Novel Meso-Alkyl Polymeric BODIPY Dyes. RSC Adv. 2013, 3 (24), 9219-9222. https://doi.org/10.1039/c3ra41298a. 
(21) Nakamura, M.; Matsuo, K.; Ito, S.; Nakamura, E. Iron-Catalyzed Cross-Coupling of Primary and Secondary Alkyl Halides with Aryl Grignard Reagents. J. Am. Chem. Soc. 2004, 126 (12), 3686-3687. https://doi.org/10.1021/ja049744t.

(22) Perkins, W.; Fischer, F. R. Inserting Porphyrin Quantum Dots in Bottom-Up Synthesized Graphene Nanoribbons. Chem. - A Eur. J. 2017, 23 (70), 1768717691. https://doi.org/10.1002/chem.201705252. 